\title{
Multiple-Spike Ground State Solutions of the Gierer-Meinhardt Equations for Biological Activator-Inhibitor Systems
}

\author{
Yuncheng You \\ Communicated by Yuncheng You, received May 11, 2005
}

\begin{abstract}
In many biological pattern formation processes and in some chemical or biochemical reactions, an activator-inhibitor system of two reactiondiffusion equations serves as a mathematical model, typically, the GiererMeinhardt equations. This type of model equations features two largely different diffusion coefficients and an essentially nonlocal nonlinearity. In this paper, the one-dimensional Gierer-Meinhardt equations are considered,

$$
\begin{aligned}
& A_{t}=d \Delta A-A+\frac{A^{2}}{H}=0 \quad \text { in } \mathbb{R}, \\
& H_{t}=D \Delta H-H+A^{2}=0 \quad \text { in } \mathbb{R},
\end{aligned}
$$$$
A, H>0 \text { and } A, H \rightarrow 0 \text { as }|x| \rightarrow \infty,
$$

where $\sigma^{2}=d / D \ll 1$. By the Lyapunov-Schmidt method, a sharp orderestimate of the number $k$ of multiple spikes of the ground state solutions is made. The $k$-spike solutions are constructed by adding small perturbation to the function which has $k$ appropriately distributed spikes resembling the solution of the problem

$$
\begin{gathered}
\Delta u-u+u^{2}=0 \text { in } \mathbb{R}, \\
0<u \rightarrow 0 \text { as }|x| \rightarrow \infty .
\end{gathered}
$$

The main result is that, for sufficiently small $\sigma>0$, there exists such a ground state solution with $k=$ const $\sigma^{-\beta}$, where $0<\beta<1 / 2$ and $\beta$ can be arbitrarily close to $1 / 2$. In the proof of this conjecture, a priori estimates of linear and nonlinear parts are conducted by means of cut-off decomposition, sharp calculations of multiple spike interactions at all levels, and finally a fine-tuned adjustment of spike centers.
\end{abstract}

\section{Contents}

1. Introduction

1991 Mathematics Subject Classification. Primary: 35B25, 35B40, 35B45; Secondary: 35J55, 92C15, 92C40.

Key words and phrases. Gierer-Meinhardt equation, multiple-spike solution, activatorinhibitor system, pattern formation, Lyapunov-Schmidt method. 
2. Main Result and Approach of Proof 195

3. The Bounded Invertibility of the Linear Operator 199

4. Nonlinear Estimates $\quad 222$

5. Solution to the Augmented Nonlocal Problem 238

6. Solution to the Reduced Finite-Dimensional Problem 247

Appendix A. $\quad 255$

Appendix B. $\quad 255$

$\begin{array}{ll}\text { Appendix C. } & 257\end{array}$

$\begin{array}{ll}\text { Acknowledgements } & 258\end{array}$

$\begin{array}{ll}\text { References } & 258\end{array}$

\section{Introduction}

In 1952, A. M. Turing [21] showed that a reaction-diffusion system modeling two chemical substances, called morphogens, could generate a spatially nonuniform pattern from a spatially almost uniform steady-state, which is stable in the absence of diffusion, by using a symmetry-breaking instability analysis driven by the distinguished diffusion coefficients. Then it has been found experimentally and numerically $[\mathbf{2}, \mathbf{7}, \mathbf{8}, \mathbf{1 2}, \mathbf{1 4}]$ that, for many reaction-diffusion systems, the ground states as well as the evolving dynamics can exhibit a common phenomenon called point condensation $[\mathbf{2 0 , 2 6 , 2 8}$, which consists of spike-type patterns with bumps around certain spatial points. Although the microscopic and/or biochemical reasons causing such phenomena of pattern formation are still under investigation in many cases, mathematicaly a coherent theory for the existence and stability of the multi-spike solutions (or called multi-bump solutions, multi-spot solutions) for several typical reaction-diffusion systems has been established based on the methods in nonlinear elliptic partial differential equations, linear stability analysis for parabolic equations, and singular perturbation analysis. Many of these results are listed in the References.

The Gierer-Meinhardt model [7] proposed in 1972 and the Gray-Scott model [8] proposed in 1983, each having certain variations in terms of the nonlinearity, are two typical models of reaction-diffusion systems which have been intensively studied by a number of authors in the last two decades, cf. $[\mathbf{1}, \mathbf{3}-\mathbf{5}, \mathbf{1 1}, \mathbf{2 3}, \mathbf{2 5}, \mathbf{2 7}, \mathbf{2 8}, \mathbf{3 0}$ $\mathbf{3 2}, \mathbf{3 5}, \mathbf{3 6}]$ for the Gierer-Meinhardt system and $[\mathbf{6}, \mathbf{1 5}, \mathbf{2 2}, \mathbf{2 6}, \mathbf{2 9}, \mathbf{3 3}, \mathbf{3 4}]$ for the Gray-Scott system.

The Gierer-Meinhardt model describes the activator-inhibitor coupled behavior for many systems in cell biology and physiology $[\mathbf{7 , 1 2}, \mathbf{1 4}]$, whereas the Gray-Scott model characterizes self-replicating patterns of some autocatalytic and feedback systems in reaction kinetics and biochemistry $[\mathbf{2}, \mathbf{6}, \mathbf{8}]$. It is interesting to note that both model systems admit specific symmetric and asymmetric multi-spike (multibump) patterns in one-dimensional and two-dimensional spatial domains, as seen in the listed references and additional papers cited therein.

Motivitated by and based on the pioneering work during 1986-1996 [13,16-20] on the profiles, especially the location and the shape of peaks, of the variational solutions to Neumann problems of semilinear elliptic PDEs, researches have been conducted for Neumann problems of single nonlinear elliptic PDEs $[\mathbf{9}, \mathbf{1 0}]$ in the 
form

$$
\epsilon^{2} \Delta u-u+f(u)=0
$$

and for Gierer-Meinhardt system and Gray-Scott system on the existence and construction of $K$-spike solutions, where $K$ is any given positive integer, by allowing the coefficient $\epsilon^{2}$ in the above equation and the ratio $\sigma^{2}$ of two largely different diffusion coefficients in Gierer-Meinhardt or Gray-Scott system to be sufficiently small, while $K$ is fixed and can be large. A common and notable feature in these

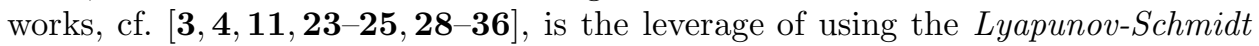
method of finite dimensional reduction to find the multi-spike profiles of ground state solutions as a small perturbation of a basis function whose multiple spikes are well shaped and distributed.

Consider a prescaled Gierer-Meinhardt system of two coupled nonlinear parabolic equations,

$$
\begin{aligned}
A_{t} & =d \Delta A-A+\frac{A^{2}}{H}, \quad x \in \Omega, t>0, \\
H_{t} & =D \Delta H-H+A^{2}, \quad x \in \Omega, t>0, \\
\frac{\partial A}{\partial n} & =\frac{\partial H}{\partial n}=0, \quad x \in \partial \Omega, t \geq 0,
\end{aligned}
$$

where $\Omega$ is a $1 \mathrm{D}$ or $2 \mathrm{D}$ bounded, Lipschitzian domain, $A=A(x, t)$ and $H=$ $H(x, t)$ are the concentrations of an activator substance and an inhibitor substance, respectively. As we know, an important thing is to study the positive, non-constant, steady states called ground states of this GM system. They are the solutions of the elliptic system:

$$
\begin{gathered}
d \Delta A-A+\frac{A^{2}}{H}=0, \quad x \in \Omega, \\
D \Delta H-H+A^{2}=0, \quad x \in \Omega, \\
\frac{\partial A}{\partial n}=\frac{\partial H}{\partial n}=0, \quad x \in \partial \Omega .
\end{gathered}
$$

In the original model in $[\mathbf{7}]$ and in many other biological settings, it is suggested or has been justified that one can make the assumption of a slowly diffusing activator and a rapidly diffusing inhibitor, which implies that $d \ll D$ and $\sigma^{2}=d / D \ll 1$.

We can rescale the independent variables and two unknowns $A$ and $H$ as follows; set

$$
u(x)=\sigma^{2} A\left(d^{1 / 2} x\right), \quad v(x)=\sigma^{2} H\left(d^{1 / 2} x\right) .
$$

Then the above elliptic system reduces to the following equivalent system,

$$
\begin{gathered}
\Delta u-u+\frac{u^{2}}{v}=0, \quad x \in \Omega_{d}, \\
\Delta v-\sigma^{2} v+u^{2}=0, \quad x \in \Omega_{d}, \\
\frac{\partial u}{\partial n}=\frac{\partial v}{\partial n}=0, \quad x \in \partial \Omega_{d} .
\end{gathered}
$$


Here, $\Omega_{d}=d^{-1 / 2} \Omega$. When $d \rightarrow 0$ but $\sigma$ remains quantitatively stabilized, for the one-dimensional domain we come up with the limiting system

$$
\begin{aligned}
& u^{\prime \prime}-u+\frac{u^{2}}{v}=0, \quad x \in \mathbb{R}, \\
& v^{\prime \prime}-\sigma^{2} v+u^{2}=0, \quad x \in \mathbb{R}, \\
& u, v>0 \text { and } u, v \rightarrow 0 \text { as }|x| \rightarrow \infty .
\end{aligned}
$$

The system (1.1) and its two-dimensional counterpart serve as a natural approximation to the Gierer-Meinhardt ground state equations when $\Omega_{d}$ is very large and a pattern formation occurs in the interior of the domain away from the boundary.

A particularly notable question concerning the pattern formation of the ground states of the Gierer-Meinhardt system (1.1) and its two-dimensional counterpart stands as follows:

Is it true that there exist solutions with an arbitrarily large number of spikes as the diffusion ratio parameter $\sigma$ gets smaller and smaller? If so, then what is the estimate of the maximal multiple spike number $k$ in terms of a sufficiently small given $\sigma$ ?

The first half of this question has been affirmatively answered by [3] for the 1D case and by $[4]$ for the $2 \mathrm{D}$ case. There are other related results in the listed references too. What they have proved is that given an arbitrarily large integer $K$, there is a

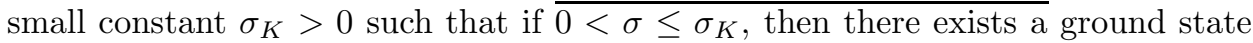
solution of (1.1), which exhibits exactly $K$ spikes in the activator component. There are a couple of different mechanisms to mathematically construct these multi-spike ground state solutions.

In this work, we shall answer the second half of the aforementioned question. Specifically, the stand point is: given an arbitrarily small $\sigma>0$, how many (namely up to what order $k$ relative to $\sigma$ ) spikes can one expect to occur in a ground state solution of the limiting Gierer-Meinhardt system? As far as we are aware, this is an open problem.

We shall take the approach of using the Lyapunov-Schmidt method to tackle this problem. The entire work consists of three stages: the linear part, the nonlinear part, and the final solution to the finite dimensional reduction. In dealing with the variable number $k$ of multiple spikes in an unknown order of power $\sigma$ as conjectured, we have to conduct sophisiticated a priori estimates in order to get the sharpest estimates in each step throughout the process of analysis.

In treating the linear part, which is the principal approximation of the Fréchet derivative of the nonlinear, nonlocal operator of the reduced single equation, a cutoff decomposition plays a key role to achieve the bounded invertibility of this linear operator. In the nonlinear part, we have to integrate all the aspects of multiple spike interactions at several levels and to assemble all the estimates together for a sharpest attempt.

In the rest of this section, we shall set up some basic facts and concepts involved in this paper. Most importantly, we shall establish several Tool Lemmas which will be very instrumental and frequently used in the subsequent sections. 
First of all, what we call spike (or bump) means a function which resembles the unique analytic solution $U(x)$ to the ODE problem:

$$
\begin{aligned}
& u^{\prime \prime}-u+u^{2}=0, \quad x \in \mathbb{R}, \\
& 0<u(x) \rightarrow 0, \quad \text { as }|x| \rightarrow \infty .
\end{aligned}
$$

We can solve (1.2) to get the explicit form of $U(x)$ (see Appendix A):

$$
U(x)=6 \operatorname{sech}^{2}\left(\frac{x}{2}\right)=\frac{6 e^{x}}{\left(1+e^{x}\right)^{2}}=\frac{6 e^{-x}}{\left(1+e^{-x}\right)^{2}}, \quad x \in \mathbb{R} .
$$

This $U(x)$ is an even, positive function. The following properties of $U$ will be used frequently. hold.

LEMma 1.1. For the spike function $U(x)$ given by (1.3), the following properties

(1) $U(x)=\frac{6 e^{-|x|}}{\left(1+e^{-|x|}\right)^{2}}, x \in \mathbb{R}$. We have

$$
\begin{aligned}
& U(x)=|U(x)| \leq 6 e^{-|x|}, \quad x \in \mathbb{R}, \\
& U(x)=6 e^{-|x|}\left(1+O\left(e^{-|x|}\right)\right), \quad \text { as }|x| \rightarrow \infty .
\end{aligned}
$$

(2) $U^{\prime}(x)=\frac{6 e^{x}\left(1-e^{x}\right)}{\left(1+e^{x}\right)^{3}}=\frac{-6 e^{-x}\left(1-e^{-x}\right)}{\left(1+e^{-x}\right)^{3}}, x \in \mathbb{R}$, and $U^{\prime}$ is an odd function,

$$
\begin{aligned}
& \left|U^{\prime}(x)\right| \leq 6 e^{-|x|}, \quad x \in \mathbb{R} \\
& \left|U^{\prime}(x)\right|=6 e^{-|x|}\left(1+O\left(e^{-|x|}\right)\right), \quad \text { as }|x| \rightarrow \infty
\end{aligned}
$$

(3) $U^{\prime \prime}(x)=\frac{6 e^{-|x|}}{\left(1+e^{-|x|}\right)^{4}}\left(1-4 e^{-|x|}+e^{-2|x|}\right), x \in \mathbb{R}$.

(4) We have

$$
\begin{aligned}
& \int_{\mathbb{R}} U(x) d x=\int_{\mathbb{R}} U^{2}(x) d x=6, \quad \int_{\mathbb{R}} U^{3}(x) d x=\frac{36}{5} \\
& \int_{\mathbb{R}}\left|U^{\prime}(x)\right| d x=3, \quad \int_{\mathbb{R}}\left|U^{\prime}(x)\right|^{2} d x=\frac{6}{5} \\
& \int_{\mathbb{R}}|x| U(x) d x=12 \log 2, \quad \int_{\mathbb{R}}|x| U^{2}(x) d x=12 \log 2-3, \\
& \int_{\mathbb{R}} e^{-|x|} U^{2}(x) d x=3, \quad \int_{\mathbb{R}} e^{-x} U^{2}(x) d x=12 .
\end{aligned}
$$

Proof. These integrals can be calculated directly. See Appendix B.

The following two lemmas are most instrumental throughout this paper, which allows us to make the sharpest estimates.

LEMMA 1.2. Let $r$ be a given real number and $p_{r}(x)$ be the function

$$
p_{r}(x)=e^{-|x|} e^{-|x+r|}, \quad x \in \mathbb{R} .
$$


Then the following properties hold:

$$
\begin{aligned}
& \left|p_{r}(x)\right|=p_{r}(x) \leq e^{-|r|}, \quad x \in \mathbb{R}, \\
& \int_{\mathbb{R}} p_{r}(x) d x=\int_{\mathbb{R}} e^{-|x|} e^{-|x+r|} d x=(1+|r|) e^{-|r|},
\end{aligned}
$$

and

$$
\begin{gathered}
\int_{\mathbb{R}}|x| p_{r}(x) d x=\int_{\mathbb{R}}|x| e^{-|x|} e^{-|x+r|} d x=\frac{1}{2} e^{-|r|}\left(1+|r|+r^{2}\right) \\
\int_{\mathbb{R}}|x|^{2} p_{r}(x) d x=\int_{\mathbb{R}}|x|^{2} e^{-|x|} e^{-|x+r|} d x=e^{-|r|}\left[\frac{1}{2}\left(|r|+r^{2}\right)+\frac{1}{3}|r|^{3}\right], \\
\int_{\mathbb{R}}|x|^{2} p_{r}(x)^{2} d x=\int_{\mathbb{R}}|x|^{2} e^{-2|x|} e^{-2|x+r|} d x=e^{-2|r|}\left(\frac{1}{16}+\frac{|r|}{8}+\frac{r^{2}}{4}+\frac{|r|^{3}}{3}\right) \\
\leq \frac{1}{3} e^{-2|r|}(1+|r|)^{3} \\
\int_{\mathbb{R}}|x| e^{-|x|} e^{-2|x+r|} d x \leq 2 e^{-|r|}\left(|r|+e^{-|r|}\right) .
\end{gathered}
$$

PROOF. The function $p_{r}(x)$ can be explicitly written as follows:

$$
\begin{aligned}
& p_{r}(x)=\left\{\begin{array}{lll}
e^{-(2 x+r)}, & x \in[0, \infty), \\
e^{-r}, & x \in[-r, 0], \\
e^{2 x+r}, & x \in(-\infty,-r],
\end{array} \quad \text { if } r>0 ;\right. \\
& p_{r}(x)=\left\{\begin{array}{lll}
e^{-(2 x+r)}, & x \in[-r, \infty), \\
e^{r}, & x \in[0,-r], \\
e^{2 x+r}, & x \in(-\infty, 0],
\end{array} \text { if } r<0 .\right.
\end{aligned}
$$

Then (1.7)-(1.10) can be validated by straightforward integration, using substitutions or integration by parts. See Appendix C for the details.

REMARK 1. Since these relevant integrals we deal with in the subsequent sections are calculated exactly in Lemma 1.2, without any possible improvement, the corresponding estimates will be deemed as sharp estimates.

Let $\beta$ be a constant, $0<\beta<1$. For sufficiently small $\sigma>0$, let

$$
r_{\sigma}=(1-\beta)|\log \sigma| \text {. }
$$

We have, for any positive integer $m$,

$$
e^{-m r_{\sigma}}=e^{-m(1-\beta)|\log \sigma|}=\sigma^{m(1-\beta)} .
$$

The following estimates on the sums of finite geometric and mixed series of uncertain length are very useful in the sequel.

LEMMA 1.3. Let $k \geq 1$ be an integer and let $0 \leq b_{j} \leq 2$ for $j=1, \ldots, k$. For $r=r_{\sigma}$ given by (1.11), we have

$$
\sum_{j=1}^{k} b_{j} e^{-j r} \leq \frac{2 \sigma^{1-\beta}}{1-\sigma^{1-\beta}},
$$

and

$$
\sum_{j=1}^{k} b_{j}(j|\log \sigma|) e^{-j r} \leq \frac{2|\log \sigma| \sigma^{1-\beta}}{\left(1-\sigma^{1-\beta}\right)^{2}}
$$


for sufficiently small $\sigma$.

Proof. (1.13) is obviously true. We show (1.14) by using Abel's transformation

$$
\sum_{i=1}^{m} a_{i} d_{i}=a_{m} D_{m}-\sum_{i=1}^{m-1}\left(a_{i+1}-a_{i}\right) D_{i}, \quad \text { with } D_{i}=\sum_{j=1}^{i} d_{j} .
$$

This is the discrete version of integration by parts. Thus we can regard $j r$ as $a_{j}$ and $e^{-j r}$ as $d_{j}$ to get

$$
\begin{aligned}
& \sum_{j=1}^{k} b_{j}(j|\log \sigma|) e^{-j r}=\sum_{j=1}^{k} \frac{b_{j}}{1-\beta}(j r) e^{-j r} \leq \frac{2}{1-\beta} \sum_{j=1}^{k} j r e^{-j r} \\
= & \left.a\left\{\frac{k r e^{-r}\left(1-e^{-k r}\right)}{1-e^{-r}}-\sum_{j=1}^{k-1} \frac{r e^{-r}\left(1-e^{-j r}\right)}{1-e^{-r}}\right\} \quad \text { (here } a=\frac{2}{1-\beta}\right) \\
\leq & a\left\{\frac{k r e^{-r}}{1-e^{-r}}-\frac{r e^{-r}}{1-e^{-r}}\left(k-1-\sum_{j=1}^{k-1} e^{-j r}\right)\right\} \\
= & a\left\{\frac{r e^{-r}}{1-e^{-r}}+\frac{r e^{-r}}{1-e^{-r}} \cdot \frac{e^{-r}\left(1-e^{-(k-1) r}\right)}{1-e^{-r}}\right\} \\
\leq & \frac{a r e^{-r}}{1-e^{-r}}\left(1+\frac{e^{-r}}{1-e^{-r}}\right)=\frac{a r e^{-r}}{\left(1-e^{-r}\right)^{2}}=\frac{2|\log \sigma| \sigma^{1-\beta}}{\left(1-\sigma^{1-\beta}\right)^{2}} .
\end{aligned}
$$

As a corollary of (1.14), for sufficiently small $\sigma$, there is a uniform constant $C_{0}>0$, such that

$$
\sum_{j=1}^{k} b_{j}[j(1+|\log \sigma|)] e^{-j r} \leq \frac{C_{0}|\log \sigma| \sigma^{1-\beta}}{\left(1-\sigma^{1-\beta}\right)^{2}} .
$$

Let integer $k \geq 2$ and $0<\beta<1$ be given. Define set in $\mathbb{R}^{k}$ as follows,

$$
=\left\{\xi=\left(\xi_{1}, \ldots, \xi_{k}\right): \begin{array}{l}
\xi_{j}<\xi_{j+1}, j=1, \ldots, k-1, \sum_{j=1}^{k} \xi_{j}=0 \\
\text { and }(1-\beta)|\log \sigma| \leq\left|\xi_{j}-\xi_{j+1}\right| \leq 1+|\log \sigma|, \\
j=1, \ldots, k-1
\end{array}\right\} .
$$

With the observation of (1.15), in the subsequent sections, we can replace the inequality $\left|\xi_{j}-\xi_{j+1}\right| \leq 1+|\log \sigma|$ by writing $\left|\xi_{j}-\xi_{j+1}\right| \leq|\log \sigma|$, just for notational simplicity, that will not affect any estimate. We shall not repeat this remark.

For any given $\xi=\left(\xi_{1}, \ldots, \xi_{k}\right) \in \Lambda$, define a corresponding multi-spike function

$$
W(x)=\sum_{j=1}^{k} U\left(x-\xi_{j}\right), \quad x \in \mathbb{R} .
$$

The partial derivatives of $W$ will be denoted by

$$
W_{j}(x)=\frac{\partial W}{\partial \xi_{j}}=-U^{\prime}\left(x-\xi_{j}\right), \quad j=1, \ldots, k .
$$


By (1.6), we have

$$
\int_{\mathbb{R}}\left|W_{j}(x)\right| d x=3 \text { and } \int_{\mathbb{R}}\left|W_{j}(x)\right|^{2} d x=\frac{6}{5}, \quad j=1, \ldots, k .
$$

The following is a known result [4].

LEMMA 1.4. The unique variational solution in $H^{1}(\mathbb{R})$ to the problem

$$
\begin{gathered}
-\varphi^{\prime \prime}+(1-2 U(x)) \varphi=0 \quad \text { in } \mathbb{R}, \\
\varphi(x) \rightarrow 0 \text { as }|x| \rightarrow \infty,
\end{gathered}
$$

is $\varphi(x)=U^{\prime}(x)$. Let $L_{1}$ be the operator $L_{1}(\varphi)=-\varphi^{\prime \prime}+(1-2 W(x)) \varphi$. Then the kernel of $L_{1}$ is

$$
\operatorname{ker} L_{1}=\operatorname{Span}\left\{W_{1}, \ldots, W_{k}\right\} \text {. }
$$

The following lemma shows that the integral in (1.20) has the same order of $k$ which affects some estimates we will encounter.

LEMMA 1.5. For any given $\xi=\left(\xi_{1}, \ldots, \xi_{k}\right) \in \Lambda$, given in (1.16), if $\mu>0$ is a constant, then

$$
\int_{\mathbb{R}} e^{-\mu \min _{1 \leq i \leq k}\left|x-\xi_{i}\right|} d x \leq \frac{2}{\mu}\left[k-(k-1) \sigma^{\mu / 2}\right],
$$

for $0<\sigma<1$.

PROOF. We can directly compute this integral to get

$$
\begin{gathered}
\int_{\mathbb{R}} e^{-\mu \min _{1 \leq i \leq k}\left|x-\xi_{i}\right|} d x=\int_{-\infty}^{\xi_{1}} e^{-\mu\left(\xi_{1}-x\right)}+\int_{\xi_{1}}^{\frac{1}{2}\left(\xi_{1}+\xi_{2}\right)} e^{-\mu\left(x-\xi_{1}\right)} \\
\quad+\int_{\frac{1}{2}\left(\xi_{1}+\xi_{2}\right)}^{\xi_{2}} e^{-\mu\left(\xi_{2}-x\right)}+\cdots+\int_{\xi_{k}}^{\infty} e^{-\mu\left(x-\xi_{k}\right)} \\
=\frac{2 k}{\mu}-\frac{2(k-1)}{\mu} e^{-\frac{\mu}{2}(1+|\log \sigma|)}=\frac{2}{\mu}\left[k-(k-1) \sigma^{\mu / 2}\right] .
\end{gathered}
$$

Let us also introduce a cut-off decomposition for functions. Define $\theta(x)$ to be a truncating function:

$$
\begin{gathered}
\qquad(x)=\theta_{\sigma}(x) \\
= \begin{cases}1, & \text { for }|x| \leq r_{\sigma} / 4, \\
\text { smooth and decreasing, } & \text { for }|x| \in\left[r_{\sigma} / 4, r_{\sigma} / 2\right], \\
0, & \text { for }|x| \geq r_{\sigma} / 2,\end{cases}
\end{gathered}
$$

where $r_{\sigma}$ is given in $(1.11)$, such that $\theta \in C^{2}(\mathbb{R})$. For small $\sigma$, we can make $\left|\theta^{\prime}(x)\right| \leq 1$. Then, for any given $\xi=\left(\xi_{1}, \ldots, \xi_{k}\right) \in \Lambda$, a function $\varphi(x)$ defined on $\mathbb{R}$ can be written as

$$
\varphi(x)=\sum_{j=0}^{k} \varphi_{j}(x)=\varphi_{0}(x)+\sum_{j=1}^{k} \varphi(x) \theta\left(x-\xi_{j}\right),
$$

namely, $\varphi_{j}(x)=\varphi(x) \theta\left(x-\xi_{j}\right), j=1, \ldots, k$. This will be referred to as the cut-off decomposition with respect to $\xi$. By the separation of the points $\xi_{1}, \ldots, \xi_{k}$ we have

$$
\operatorname{supp} \varphi_{i} \cap \operatorname{supp} \varphi_{j}=\emptyset, \quad \text { for } i \neq j \text { in }\{1, \ldots, k\} \text {. }
$$


Let

$$
\Omega_{0}=\mathbb{R} \backslash \bigcup_{j=1}^{k} \Omega_{j}, \quad \Omega_{j}=\left[\xi_{j}-\frac{r_{\sigma}}{4}, \xi_{j}+\frac{r_{\sigma}}{4}\right], \quad j=1, \ldots, k .
$$

Then $\operatorname{supp} \varphi_{0} \subset \Omega_{0}$ and

$$
\Omega=\bigcup_{j=0}^{k} \Omega_{j}
$$

\section{Main Result and Approach of Proof}

We use $\Delta$ to denote the second-order distributional derivative of a function defined on $\mathbb{R}$, namely, $\Delta \varphi=\varphi^{\prime \prime}$. We shall use $I$ to denote the identity operator. Consider the linear differential operator

$$
\begin{gathered}
L_{o}(\varphi)=\left(-\Delta+\sigma^{2} I\right)(\varphi), \quad \varphi \in H^{2}(\mathbb{R}) \cap H_{0}^{1}(\mathbb{R}), \\
0<\varphi(x) \rightarrow 0, \quad \text { as }|x| \rightarrow \infty .
\end{gathered}
$$

The Green's function associated with $L_{o}$ is found to be

$$
G(x, y)=\frac{1}{2 \sigma} e^{-\sigma|x-y|}, \quad \text { for } x, y \in \mathbb{R} .
$$

Its verification is straightforward and omitted.

First of all, we can rescale the problem (1.1). Let $\tau>0$ be a parameter, which will be specified later. We can replace $u$ and $v$ in (1.1) by the rescaled $\tau u$ and $\tau v$, then (1.1) becomes the problem

$$
\begin{gathered}
u^{\prime \prime}-u+u^{2} / v=0, \quad \text { in } \mathbb{R} \\
v^{\prime \prime}-\sigma^{2} v+\tau u^{2}=0, \\
u, v>0 \text { and } u, v \rightarrow 0 \text { as }|x| \rightarrow \infty .
\end{gathered}
$$

Since $L_{o}: H^{2}(\mathbb{R}) \cap H_{0}^{1}(\mathbb{R}) \rightarrow L^{2}(\mathbb{R})$ is invertible and its inverse operator is bounded, the second ODE in the system (2.3) can be solved for $v$, and we have

$$
v=T\left[u^{2}\right] \triangleq L_{0}^{-1}\left(\tau u^{2}\right)=\left(-\Delta+\sigma^{2} I\right)^{-1}\left(\tau u^{2}\right)
$$

By substituting (2.4) into the first ODE in (2.3), the rescaled problem (2.3) is reduced to a single nonlocal nonlinear ODE problem:

$$
\begin{gathered}
S[u] \triangleq u^{\prime \prime}-u+\frac{u^{2}}{T\left[u^{2}\right]}=0 \quad \text { in } \mathbb{R}, \\
u>0 \text { and } u \rightarrow 0 \text { as }|x| \rightarrow \infty .
\end{gathered}
$$

The operator $S$ is Fréchet differentiable. In order to distinguish the solution to the original problem (1.1) and the solution to the rescaled problems (2.3) and (2.5), we shall retain the notation $(u, v)$ for $(2.3)$ and $(2.5)$ and denote the solution to (1.1) by $\left(u^{*}, v^{*}\right)$.

We take a normalization parameter $\tau$ to be

$$
\tau=\tau_{\sigma}=\frac{2 \sigma}{\omega}, \quad \text { where } \omega=\int_{\mathbb{R}} W^{2}(x) d x,
$$

where $W(x)$ is the multi-spike function given in (1.17).

In this work, we shall prove the following main result, Theorem 2.1. 
THEOREM 2.1. There exists a positive number $\sigma_{0}$ such that for any given $\sigma$, $0<\sigma \leq \sigma_{0}$, there exists a solution $(u, v)$ to the one-dimensional Gierer-Meinhardt system (2.3) with the following properties.

(1) The u component has $k$ spikes, with

$$
k=\operatorname{const} \sigma^{-\beta} \text {, }
$$

where $0<\beta<1 / 2$ can be arbitrarily close to $1 / 2$,

and such that

$$
\lim _{\sigma \rightarrow 0}\left|u(x)-\sum_{j=1}^{k} U\left(x-\xi_{j}\right)\right|=0, \quad \text { uniformly in } x \in \mathbb{R} .
$$

(2) The $v$ component satisfies

$$
\begin{aligned}
& \quad \lim _{\sigma \rightarrow 0}\left|v\left(\xi_{j}+x\right)-1\right|=0 \text {, uniformly on any compact set of } x, \\
& \text { for } j=1, \ldots, k \text {. }
\end{aligned}
$$

Here $\xi=\left(\xi_{1}, \ldots, \xi_{k}\right)$ is some vector point in the set $\Lambda$ defined by (1.16). Moreover, the corresponding multi-spike ground state solution to the original Gierer-Meinhardt system (1.1) exists and is given by $\left(u^{*}, v^{*}\right)=(\tau u, \tau v)$.

The rest of the paper is devoted to proving this main result. Our goal is to reach a sharp estimate on the order of the multiple spike number $k$ in terms of $\sigma$. Here let us lay out the approach of the proof.

Since the problem (2.3) has been reduced to a single ODE problem (2.5), our strategy is to seek for a solution of (2.5) in the form

$$
u(x)=W(x)+\varphi(x), \quad x \in \mathbb{R},
$$

where $W$ is the function of multiple standard spikes introduced in (1.17), and $\varphi$ ought to be a small perturbation function, which tends to zero as $\sigma \rightarrow 0$ with respect to an appropriate norm.

Let $V(x)$ be the unique solution of the ODE problem:

$$
\begin{gathered}
V^{\prime \prime}-\sigma^{2} V+\tau W^{2}=0, \quad x \in \mathbb{R}, \\
0<V(x) \rightarrow 0, \text { as }|x| \rightarrow \infty .
\end{gathered}
$$

According to (2.2) and (2.4), $V=T\left[W^{2}\right]$ is expressed as

$$
V(x)=\frac{\tau}{2 \sigma} \int_{\mathbb{R}} e^{-\sigma|x-y|} W^{2}(y) d y=\frac{1}{\omega} \int_{\mathbb{R}} e^{-\sigma|x-y|} W^{2}(y) d y,
$$

where $\omega=\int_{\mathbb{R}} W^{2}(y) d y$ is shown in (2.6). When we substitute $u=W+\varphi$ into (2.5), it becomes

$$
\left(W^{\prime \prime}-W\right)+\left(\varphi^{\prime \prime}-\varphi\right)+\frac{W^{2}}{V}+\frac{2 W \varphi}{V}-\frac{2 W^{2}}{V^{2}} T[W \varphi]+(\text { r.t. })=0,
$$

where (r.t.) stands for all the remainder terms of the order $O\left(\varphi^{2}\right)$ as $\varphi$ small. Thus $\varphi$ satisfies the following equation

$$
-\varphi^{\prime \prime}+\varphi-\frac{2 W \varphi}{V}+\frac{2 W^{2}}{V^{2}} T[W \varphi]=S[W]+N(W, \varphi),
$$


where the left-hand side is a linear nonlocal operator on $\varphi$, and the right-hand side consists of nonlinear terms

$$
\begin{gathered}
S[W]=W^{\prime \prime}-W+\frac{W^{2}}{V}, \text { and } \\
N(W, \varphi)=(\text { r.t. })=\frac{(W+\varphi)^{2}}{T\left[(W+\varphi)^{2}\right]}-\left(\frac{W^{2}}{V}+\frac{2 W \varphi}{V}-\frac{2 W^{2}}{V^{2}} T[W \varphi]\right) .
\end{gathered}
$$

We shall take the approach of the Lyapunov-Schmidt method to show the existence of a solution $\varphi$ of this equation (2.14), which has the properties we demand. As for the specific procedure, we divide the entire proof into the following stages.

Stage 1. First we shall study the linear part in equation (2.14). But instead of its full form, we will consider the following linear operator, which we call the principal approximation of the linear part in (2.14),

$$
\begin{aligned}
L(\varphi) & \triangleq-\varphi^{\prime \prime}+\varphi-2 W \varphi+\frac{2 W^{2}}{\omega}\langle W, \varphi\rangle \\
& =-\varphi^{\prime \prime}+(1-2 W) \varphi+\frac{2 W^{2}}{\omega}\langle W, \varphi\rangle, \quad \varphi \in H^{2}(\mathbb{R}) \cap H_{0}^{1}(\mathbb{R}),
\end{aligned}
$$

where $\omega=\int_{\mathbb{R}} W^{2}(x) d x$ and $\langle\cdot, \cdot\rangle$ is the $L^{2}$ inner product. Comparing $L$ with the linear operator on the left side of (2.14), we have made two simplifications in (2.16) but preserved all the essentials in the linear part. These two simplifications are: in (2.16),

$$
\text { we replace } V(x) \text { by } 1 \text { and } T[W \varphi] \text { by } \frac{1}{\omega}\langle W, \varphi\rangle \text {. }
$$

In Section 3, we shall consider the following problem: Given a function $h$ whose weighted $L^{\infty}$ norm is finite, and given a set of points $\left(\xi_{1}, \ldots, \xi_{k}\right) \in \Lambda$, find a solution $\varphi$ and $\gamma=\left(\gamma_{1}, \ldots, \gamma_{k}\right) \in \mathbb{R}^{k}$ such that

$$
\begin{aligned}
& L(\varphi)=h+\gamma \cdot \nabla_{\xi} W=h+\sum_{j=1}^{k} \gamma_{j} W_{j} \text { in } \mathbb{R}, \\
& \varphi(x) \rightarrow 0 \text { as }|x| \rightarrow \infty, \text { and } \\
& \left\langle\varphi, W_{j}\right\rangle=0, \text { for } j=1, \ldots, k .
\end{aligned}
$$

Under the assumption we made in Theorem 2.1, we shall prove the solution operator is a bounded linear operator with respect to a weighted $L^{\infty}$ space by using the $a$ priori estimates, the cut-off technique, and the Fredholm alternative principle.

STAGE 2. In Section 4, we shall estimate the cost of the replacements made in (2.17), which essentially proves the statement in the main theorem (Theorem 2.1) on $v$ component. Moreover, we shall establish a key result on the estimate of integrals

$$
\int_{\mathbb{R}} S[W](x) U^{\prime}\left(x-\xi_{j}\right) d x, \quad j=1, \ldots, k,
$$

which constitutes the main part of the nonlinear side in (2.14) under investigation. We also want to estimate the weighted $L^{\infty}$ norm of $S[W]$. 
StAGE 3. By the Lyapunov-Schmidt method, the problem of seeking for a solution $\varphi$ of the original equation (2.14) is imbedded into an augmented problem of solving the problem:

$$
\begin{aligned}
& S\left[W+\varphi_{\sigma, \xi}\right](x)=\sum_{j=0}^{k} \gamma_{j} U^{\prime}\left(x-\xi_{j}\right), \quad x \in \mathbb{R}, \\
& \left\langle\varphi_{\sigma, \xi}, U^{\prime}\left(\cdot-\xi_{j}\right)\right\rangle=0, \quad j=1, \ldots, k, \text { and } \varphi_{\sigma, \xi}(x) \rightarrow 0 \text { as }|x| \rightarrow \infty,
\end{aligned}
$$

for both $\varphi_{\sigma, \xi}$ and $\gamma=\left(\gamma_{1}, \ldots, \gamma_{k}\right)$. This augmented problem is equivalent to solving

$$
\begin{aligned}
& L(\varphi)=S[W]+P(W, \varphi)+\sum_{j=1}^{k} \gamma_{j} W_{j} \\
& \left\langle\varphi, U^{\prime}\left(\cdot-\xi_{j}\right)\right\rangle=0, \quad j=1, \ldots, k \\
& \varphi(x) \rightarrow 0, \quad \text { as }|x| \rightarrow \infty,
\end{aligned}
$$

where $L$ is the linear operator of the principal approximation studied in Section 3, and the nonlinear part $P(W, \varphi)$ is given by

$$
P(W, \varphi) \triangleq \frac{(W+\varphi)^{2}}{T\left[(W+\varphi)^{2}\right]}-\left(\frac{W^{2}}{V}+2 W \varphi-\frac{2 W^{2}}{\omega}\langle W, \varphi\rangle\right) .
$$

In Section 5, we shall make estimates of the nonlinear terms in $P(W, \varphi)$. Then we shall use the contraction mapping argument and the fixed point theory to show that there exists a solution $\left(\varphi_{\sigma, \xi}, \gamma\right)$ of the problem $(2.20)$ under the assumptions we make. Furthermore, an estimate on the integrals

$$
\int_{\mathbb{R}}\left(S\left[W+\varphi_{\sigma, \xi}\right](x)-S[W](x)\right) U^{\prime}\left(x-\xi_{j}\right) d x, \quad j=1, \ldots, k,
$$

will be made as a preparation for the final stage.

STAGE 4. Now the problem is reduced to solving the finite-dimensional problem

$$
\begin{gathered}
\sum_{j=1}^{k} \gamma_{j} \int_{\mathbb{R}} U^{\prime}\left(x-\xi_{j}\right) U^{\prime}\left(x-\xi_{\ell}\right) d x=\int_{\mathbb{R}} S[W] U^{\prime}\left(x-\xi_{\ell}\right) d x \\
+\int_{\mathbb{R}}\left(S\left[W+\varphi_{\sigma, \xi}\right]-S[W]\right) U^{\prime}\left(x-\xi_{\ell}\right) d x \\
\ell=1, \ldots, k,
\end{gathered}
$$

in the sense that there exists a choice $\xi=\left(\xi_{1}, \ldots, \xi_{k}\right) \in \Lambda$ such that the right-hand side of (2.22) turns out to be zero, so that the component $\gamma_{\sigma, \xi}=\left(\gamma_{1}, \ldots, \gamma_{k}\right)$ of the solution of the augmented problem (2.19) or, equivalently, (2.20), is a zero vector corresponding to this $\xi$. Hence the linear coefficient matrix of $\gamma$ is nonsingular for small $\sigma$. This proof will be fulfilled by using the Brouwer fixed point theorem. Finally, since there exists a $\xi \in \Lambda$ which makes $\gamma_{\sigma, \xi}=0$, then the solution $\varphi_{\sigma, \xi}$ to this augmented problem (2.19) is exactly the solution of the problem

$$
S\left[W+\varphi_{\sigma, \xi}\right]=0 .
$$

Hence there exists a solution $u=W+\varphi_{\sigma, \xi}$ to the original problem (2.5), which has the property stated in Theorem 2.1 . 


\section{The Bounded Invertibility of the Linear Operator}

Here we study the linear operator of the principal approximation $L$ defined by

$$
\begin{aligned}
& L(\varphi) \triangleq-\varphi^{\prime \prime}+(1-2 W) \varphi+\frac{2 W^{2}}{\omega}\langle W, \varphi\rangle, \quad \varphi \in H^{2}(\mathbb{R}) \cap H_{0}^{1}(\mathbb{R}), \\
& \text { where } \omega=\int_{\mathbb{R}} W^{2}(x) d x
\end{aligned}
$$

as we mentioned in (2.16). The formal adjoint operator of $L$ with respect to the $L^{2}$ inner product is given by

$$
L^{*}(\varphi) \triangleq-\varphi^{\prime \prime}+(1-2 W) \varphi+\frac{2 W}{\omega}\left\langle W^{2}, \varphi\right\rangle, \quad \varphi \in H^{2}(\mathbb{R}) \cap H_{0}^{1}(\mathbb{R}) .
$$

In the sequel, we shall always refer the set $\Lambda$ to (1.16) and make the asumption (2.7), which will not be repeated in each lemma and theorem. We may only mention the assumption in Theorem 2.1 for this. We shall denote a positive constant without need of specification by a generic $C$.

First we estimate

$$
\begin{aligned}
L\left(W_{j}\right)= & L\left(-U^{\prime}\left(\cdot-\xi_{j}\right)\right)=2\left(W(x)-U\left(x-\xi_{j}\right)\right) U^{\prime}\left(x-\xi_{j}\right) \\
& -\frac{2 W^{2}}{\omega}\left\langle W, U^{\prime}\left(\cdot-\xi_{j}\right)\right\rangle \\
= & \sum_{i(\neq j)=1}^{k} 2 U\left(x-\xi_{i}\right) U^{\prime}\left(x-\xi_{j}\right)-\frac{2 W^{2}(x)}{\omega}\left\langle W, U^{\prime}\left(\cdot-\xi_{j}\right)\right\rangle,
\end{aligned}
$$

where

$$
\begin{aligned}
\mid \sum_{i(\neq j)=1}^{k} 2 U(x- & \left.\xi_{i}\right) U^{\prime}\left(x-\xi_{j}\right)\left|\leq 2 \sum_{i \neq j} U(y)\right| U^{\prime}\left(y+\xi_{i}-\xi_{j}\right) \mid \quad(\text { by Lemma 1.1) } \\
& \leq 72 \sum_{i \neq j} e^{-|y|} e^{-\left|y+\xi_{i}-\xi_{j}\right|} \leq 72 \sum_{i \neq j} e^{-\left|\xi_{i}-\xi_{j}\right|} \quad \text { (by Lemma 1.2) } \\
& \leq 72 \sum_{i \neq j} \sigma^{|i-j|(1-\beta)} \quad(\text { by }(1.12)) \\
& \leq 144 \frac{\sigma^{1-\beta}}{1-\sigma^{1-\beta}} \leq 288 \sigma^{1-\beta}
\end{aligned}
$$

where, and hereafter, we assume that $\sigma$ is so small that

$$
1-\sigma^{1-\beta}>1 / 2 \text {. }
$$

Moreover, we have

$$
\left|-\frac{2 W^{2}(x)}{\omega}\left\langle W, U^{\prime}\left(\cdot-\xi_{j}\right)\right\rangle\right|
$$




$$
\begin{gathered}
=\frac{2}{\omega}\left(\sum_{j=1}^{k} U^{2}\left(x-\xi_{j}\right)+\sum_{\substack{i, j \\
i \neq j}} U\left(x-\xi_{i}\right) U\left(x-\xi_{j}\right)\right) \\
\times \sum_{i(\neq j)=1}^{k}\left|\int_{\mathbb{R}} U\left(y-\xi_{i}\right) U^{\prime}\left(y-\xi_{j}\right) d y\right|
\end{gathered}
$$

in which,

$$
\begin{gathered}
\omega=\int_{\mathbb{R}} W^{2}(x) d x \geq \sum_{i=1}^{k} \int_{\mathbb{R}} U^{2}\left(x-\xi_{i}\right) d x=6 k, \\
\sum_{j=1}^{k} U^{2}\left(x-\xi_{j}\right)=U^{2}\left(x-\xi_{j o}\right)+\sum_{j \neq j o} U^{2}\left(x-\xi_{j}\right) \\
\leq 36+\frac{2 \sigma^{2(1-\beta)}}{1-\sigma^{2(1-\beta)}} \leq 36+4 \sigma^{2(1-\beta)}
\end{gathered}
$$

where $\min _{1 \leq j \leq k}\left|x-\xi_{j}\right|=\left|x-\xi_{j o}\right|$, and

$$
\sum_{\substack{i, j=1 \\ i \neq j}} U\left(x-\xi_{i}\right) U\left(x-\xi_{j}\right) \leq 36 \sum_{\substack{i, j \\ i \neq j}} \sigma^{|i-j|(1-\beta)} \leq 36 k\left(4 \sigma^{1-\beta}\right) \leq C \sigma^{1-2 \beta}
$$

Besides we have

$$
\begin{aligned}
& \sum_{i(\neq j)=1}^{k}\left|\int_{\mathbb{R}} U\left(y-\xi_{i}\right) U^{\prime}\left(y-\xi_{j}\right) d y\right| \leq \sum_{i \neq j}\left(1+\left|\xi_{i}-\xi_{j}\right|\right) e^{-\left|\xi_{i}-\xi_{j}\right|} \\
& \quad(\text { by Lemma 1.2) } \\
\leq & \sum_{i \neq j}\left(1+\frac{1}{1-\beta}|i-j|(1-\beta)|\log \sigma|\right) \sigma^{|i-j|(1-\beta)} \quad \text { (by Lemma 1.3) } \\
\leq & 4 \sigma^{1-\beta}+8|\log \sigma| \sigma^{1-\beta} .
\end{aligned}
$$

Then we get

$$
\begin{gathered}
\left|-\frac{2 W^{2}(x)}{\omega}\left\langle W, U^{\prime}\left(\cdot-\xi_{j}\right)\right\rangle\right| \leq \frac{1}{3 k}\left(36+C \sigma^{1-2 \beta}\right)(4+8|\log \sigma|) \sigma^{1-\beta} \\
\leq C(1+|\log \sigma|) \sigma, \quad \text { for some constant } C>0
\end{gathered}
$$

because $k=C \sigma^{-\beta}$. It follows from (3.3), (3.4) and (3.5) that

$$
\left|L\left(W_{j}\right)\right| \leq 288 \sigma^{1-\beta}+C(1+|\log \sigma|) \sigma .
$$


Similarly, we have

$$
\begin{aligned}
& \mid-\frac{2 W(x)}{\omega}\left\langle W^{2}, U^{\prime}\left(\cdot-\xi_{j}\right)\right\rangle\left|\leq \frac{1}{3 k}\left(6+4 \sigma^{1-\beta}\right)\right| \int_{\mathbb{R}} W^{2}(x) U^{\prime}\left(x-\xi_{j}\right) d x \mid \\
& \leq \frac{1}{3 k}\left(6+4 \sigma^{1-\beta}\right) \int_{\mathbb{R}}|W(x)| \sum_{i(\neq j)=1}^{k} U\left(x-\xi_{i}\right)\left|U^{\prime}\left(x-\xi_{j}\right)\right| d x \\
& \leq \frac{1}{3 k}\left(6+4 \sigma^{1-\beta}\right)^{2}\left(4 \sigma^{1-\beta}+8|\log \sigma|\right) \sigma^{1-\beta} \\
& \leq \tilde{C}(1+|\log \sigma|) \sigma, \text { for some constant } \tilde{C}>0 .
\end{aligned}
$$

Then from (3.2), (3.4) and (3.9) it follows that

$$
\left|L^{*}\left(W_{j}\right)\right| \leq 288 \sigma^{1-\beta}+\tilde{C}(1+|\log \sigma|) \sigma .
$$

Thus we have proved the following lemma.

LEMMA 3.1. Under the assumption of Theorem 2.1, it holds that

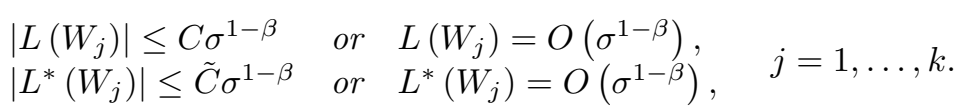

Proof. (3.11) follows from (3.8) and (3.10), since $0<\beta<1$, the term $(1+$ $|\log \sigma|) \sigma$ is dominated by the $\sigma^{1-\beta}$ term when $\sigma$ is sufficiently small.

For a fixed $0<\mu \leq \frac{1}{3}$, define $L_{\mu}^{\infty}=L_{\mu}^{\infty}(\mathbb{R})$ to be the weighted space:

$$
\begin{gathered}
L_{\mu}^{\infty}=\left\{\psi \in H_{0}^{1}(\mathbb{R}): \limsup _{|x| \rightarrow \infty} e^{\mu|x|}|\psi(x)|<\infty\right\}, \\
\text { with the norm }\|\psi\|_{\mu}=\sup \left\{e^{\mu \min _{1 \leq i \leq k}\left|x-\xi_{i}\right|}|\psi(x)|\right\} .
\end{gathered}
$$

Note that once $k$ and $\left(\xi_{1}, \ldots, \xi_{k}\right)$ are fixed, $L_{\mu}^{\infty}$ is a Banach space. Moreover, for a given $k$ and different $\xi=\left(\xi_{1}, \ldots, \xi_{k}\right) \in \Lambda$, the norms $\|\cdot\|_{\mu}$ are all equivalent.

Now consider the following problem: Given a function $h \in L_{\mu}^{\infty}\left(\subset L^{2}(\mathbb{R})\right)$ and a $\xi \in \Lambda$, find a solution $(\varphi, \gamma) \in L_{\mu}^{\infty} \times \mathbb{R}^{k}, \gamma=\left(\gamma_{1}, \ldots, \gamma_{k}\right)$, of the following problem,

$$
\begin{aligned}
& L(\varphi)=h+\gamma \cdot \nabla_{\xi} W=h+\sum_{j=1}^{k} \gamma_{j} W_{j} \text { in } \mathbb{R}, \\
& \left\langle\varphi, W_{j}\right\rangle=0, \quad j=1, \ldots, k, \text { and } \\
& \varphi(x) \rightarrow 0 \text { as }|x| \rightarrow \infty .
\end{aligned}
$$

We address the property of such a vector $\gamma=\left(\gamma_{1}, \ldots, \gamma_{k}\right)$ which satisfies equation (3.13) in the following lemma.

LEMMA 3.2. Suppose $\varphi \in L_{\mu}^{\infty}$ and $\gamma=\left(\gamma_{1}, \ldots, \gamma_{k}\right)$ satisfy equation (3.13), where $h \in L_{\mu}^{\infty}$ is given. Then it holds that, for $j=1, \ldots, k$,

$$
\left|\gamma_{j}\right| \leq\|\gamma\|_{\mathbb{R}^{k}} \leq C_{1}\|h\|_{\mu}+C_{2}(\sigma)\|\varphi\|_{\mu}
$$

where $C_{1}$ and $C_{2}(\sigma)$ are positive constants, and $C_{2}(\sigma)=O\left(\sigma^{1-\frac{3 \beta}{2}}\right)$. 
Proof. Taking the $L^{2}$ inner product of (3.13) with each $W_{j}$, we can get $(3.15)$

$$
\left\langle\varphi, L^{*}\left(W_{j}\right)\right\rangle=\left\langle h, W_{j}\right\rangle+\gamma_{j} \int_{\mathbb{R}}\left|W_{j}(x)\right|^{2} d x+\sum_{i(\neq j)=1}^{k} \gamma_{i}\left\langle W_{i}, W_{j}\right\rangle, j=1, \ldots, k .
$$

From Lemma 1.1, we know $\int_{\mathbb{R}}\left|W_{j}\right|^{2} d x=6 / 5$. By Lemma 3.1, we have

$$
\left|\left\langle\varphi, L^{*}\left(W_{j}\right)\right\rangle\right| \leq \int_{\mathbb{R}} \tilde{C} \sigma^{1-\beta} e^{-\mu \min _{1 \leq i \leq k}\left|x-\xi_{i}\right|} d x\|\varphi\|_{\mu} \leq C \sigma^{1-\beta}\|\varphi\|_{\mu}
$$

We also have

$$
\begin{gathered}
\left|\left\langle h, W_{j}\right\rangle\right| \leq 6 \int_{\mathbb{R}} e^{-\mu \min _{1 \leq i \leq k}\left|x-\xi_{i}\right|} e^{-\left|x-\xi_{j}\right|} d x\|h\|_{\mu} \\
\leq 6\|h\|_{\mu}\left\{\int_{\left|x-\xi_{j}\right| \leq r_{\sigma}} e^{-\mu \min _{1 \leq i \leq k}\left|x-\xi_{i}\right|} d x+\left(\int_{\mathbb{R}} e^{-\mu \min _{1 \leq i \leq k}\left|x-\xi_{i}\right|} d x\right) \sigma^{1-\beta}\right\}
\end{gathered}
$$

For $i \neq j$, we find that by Lemma 1.2 ,

$$
\begin{aligned}
\left|\left\langle W_{i}, W_{j}\right\rangle\right| & \leq \int_{\mathbb{R}} 36 e^{-|x|} e^{-\left|x+\xi_{i}-\xi_{j}\right|} d x \leq 36\left(1+\left|\xi_{i}-\xi_{j}\right|\right) e^{-\left|\xi_{i}-\xi_{j}\right|} \\
& \leq 36(1+|i-j||\log \sigma|) \sigma^{(1-\beta)|i-j|} .
\end{aligned}
$$

Since $(3.15)$ can be written as a linear system with respect to $\gamma_{j}$ 's,

$$
\left(\frac{6}{5} I+A\right) \gamma=\left(\begin{array}{c}
\left\langle\varphi, L^{*}\left(W_{1}\right)\right\rangle-\left\langle h, W_{1}\right\rangle \\
\vdots \\
\left\langle\varphi, L^{*}\left(W_{k}\right)\right\rangle-\left\langle h, W_{k}\right\rangle
\end{array}\right)
$$

and the matrix $A$ is given by

$$
A=\left(\begin{array}{cccc}
0 & \left\langle W_{2}, W_{1}\right\rangle & \cdots & \left\langle W_{k}, W_{1}\right\rangle \\
\left\langle W_{1}, W_{2}\right\rangle & 0 & \cdots & \left\langle W_{k}, W_{2}\right\rangle \\
\left\langle W_{1}, W_{k}\right\rangle & \left\langle W_{2}, W_{k}\right\rangle & \cdots & \left\langle W_{k}, W_{k}\right\rangle
\end{array}\right)
$$

whose operator norm relative to $\mathbb{R}^{k}$ satisfies

$$
\begin{aligned}
\|A\| & \leq \sqrt{k} \max _{i \neq j}\left|\left\langle W_{i}, W_{j}\right\rangle\right| \leq 36 \sqrt{k}(1+|\log \sigma|) \sigma^{1-\beta} \\
& \leq C(1+|\log \sigma|) \sigma^{1-\frac{3 \beta}{2}}
\end{aligned}
$$

We see that for $\sigma$ small, the matrix $(6 / 5) I+A$ is boundedly invertible. Let

$$
C_{A}=\left\|\left(\frac{6}{5} I+A\right)^{-1}\right\|
$$


Then solving (3.16), we obtain

$$
\begin{aligned}
\|\gamma\|_{\mathbb{R}^{k}} & \leq C_{A}\left\|\operatorname{col}\left(\left\langle\varphi, L^{*}\left(W_{j}\right)\right\rangle-\left\langle h, W_{j}\right\rangle\right)\right\|_{\mathbb{R}^{k}} \\
& \leq C_{A}\left[\sum_{j=1}^{k}\left(\left|\left\langle\varphi, L^{*}\left(W_{j}\right)\right\rangle\right|+\left|\left\langle h, W_{j}\right\rangle\right|\right)^{2}\right]^{1 / 2} \\
& \leq C_{A}\left\{\sqrt{k} C \sigma^{1-\beta}\|\varphi\|_{\mu}+36\left(\int_{\mathbb{R}} e^{-\mu \min _{1 \leq i \leq k}\left|x-\xi_{i}\right|} d x\right)\|h\|_{\mu}\right. \\
& \left.+12 \sqrt{k}\left(\int_{\mathbb{R}} e^{-\mu \min _{1 \leq i \leq k}\left|x-\xi_{i}\right|} d x\right) \sigma^{1-\beta}\|h\|_{\mu}\right\} \\
& \leq C_{1}\|h\|_{\mu}+C_{2}(\sigma)\|\varphi\|_{\mu}
\end{aligned}
$$

where

$$
\begin{aligned}
& C_{1}=C_{A}\left(36+12 C \sigma^{1-\frac{3 \beta}{2}}\right) \int_{\mathbb{R}} e^{-\mu \min _{1 \leq i \leq k}\left|x-\xi_{i}\right|} d x, \\
& C_{2}(\sigma)=C_{A} C \sigma^{1-\frac{3 \beta}{2}}
\end{aligned}
$$

Thus, the lemma is proved.

As a corollary, if (3.13) admits a unique solution $(\varphi, \gamma)$ such that

$$
\|\varphi\|_{\mu} \leq \mathrm{const}\|h\|_{\mu}
$$

then $\gamma=\left(\gamma_{1}, \ldots, \gamma_{k}\right)$ is uniquely determined by (3.16) and satisfies

$$
\|\gamma\|_{\mathbb{R}^{k}} \leq \mathrm{const}\|h\|_{\mu}
$$

provided that $\beta \leq 2 / 3$.

Define a self-adjoint linear operator

$$
L_{1}=-\Delta+(1-2 W) I: H^{2}(\mathbb{R}) \cap H_{0}^{1}(\mathbb{R}) \rightarrow L^{2}(\mathbb{R}),
$$

which is a part of the linear operator $L$ in (3.1). Set

$$
Z(x)=\sum_{j=1}^{k}\left(x-\xi_{j}\right) U^{\prime}\left(x-\xi_{j}\right)+2 W(x) .
$$


We have

$$
\begin{aligned}
& L_{1}(Z)=(-\Delta+(1-2 W))\left(\sum_{j=1}^{k}\left(x-\xi_{j}\right) U^{\prime}\left(x-\xi_{j}\right)+2 W(x)\right) \\
= & -\left(\sum_{j} U^{\prime}\left(x-\xi_{j}\right)+\sum_{j}\left(x-\xi_{j}\right) U^{\prime \prime}\left(x-\xi_{j}\right)\right)^{\prime} \\
& -2 W^{\prime \prime}+(1-2 W)\left(\sum_{j}\left(x-\xi_{j}\right) U^{\prime}\left(x-\xi_{j}\right)+2 W(x)\right) \\
= & -4 W^{\prime \prime}+(1-2 W) 2 W+\sum_{j}\left(x-\xi_{j}\right)\left[-U^{\prime \prime \prime}\left(x-\xi_{j}\right)+U^{\prime}\left(x-\xi_{j}\right)\right. \\
- & \left.2 W U^{\prime}\left(x-\xi_{j}\right)\right] \\
= & -2 W-4 \sum_{\substack{i, j \\
i \neq j}} U\left(x-\xi_{i}\right) U\left(x-\xi_{j}\right)-2 \sum_{\substack{i, j \\
i \neq j}}\left(x-\xi_{j}\right) U\left(x-\xi_{i}\right) U^{\prime}\left(x-\xi_{j}\right),
\end{aligned}
$$

where

$$
\begin{aligned}
& \left\|-4 \sum_{\substack{i, j \\
i \neq j}} U\left(x-\xi_{i}\right) U\left(x-\xi_{j}\right)\right\|_{L^{2}} \leq 144 \sum_{\substack{i, j \\
i \neq j}}\left(\int_{\mathbb{R}} e^{-2|x|} e^{-2\left|x+\xi_{i}-\xi_{j}\right|} d x\right)^{1 / 2} \\
& =144 \sum_{i \neq j}\left(\left[\frac{1}{2}+\left|\xi_{i}-\xi_{j}\right|\right] e^{-2\left|\xi_{i}-\xi_{j}\right|}\right)^{1 / 2} \\
& \leq 144 \sum_{i \neq j}\left(1+\left|\xi_{i}-\xi_{j}\right|^{1 / 2}\right) e^{-\left|\xi_{i}-\xi_{j}\right|} \\
& \leq 144 \sum_{i \neq j}(1+\sqrt{|i-j||\log \sigma|}) \sigma^{|i-j|(1-\beta)} \\
& \leq 576 k \sigma^{1-\beta}+288\left(\sum_{i \neq j}|i-j||\log \sigma| \sigma^{2|i-j|(1-\beta)}\right)^{1 / 2} \quad(\text { by Lemma 1.3) } \\
& \leq C \sigma^{1-2 \beta}+C \sqrt{k} \sigma^{1-\beta}|\log \sigma|^{1 / 2} \leq C \sigma^{1-2 \beta}+C \sigma^{1-\frac{3 \beta}{2}}|\log \sigma|^{1 / 2} \\
& =O\left(\sigma^{1-2 \beta}\right)+O\left(\sigma^{1-\frac{3 \beta}{2}}|\log \sigma|^{1 / 2}\right),
\end{aligned}
$$


and

$$
\begin{aligned}
& \left\|-2 \sum_{\substack{i, j \\
i \neq j}}\left(x-\xi_{j}\right) U\left(x-\xi_{i}\right) U^{\prime}\left(x-\xi_{j}\right)\right\|_{L^{2}} \\
& \leq 72 \sum_{i \neq j}\left\{\int_{\mathbb{R}}|x|^{2} e^{-2|x|} e^{-2\left|x+\xi_{j}-\xi_{i}\right|} d x\right\}^{1 / 2} \quad \text { (by using (1.9) in Lemma 1.2) } \\
& \leq \frac{72}{\sqrt{3}} \sum_{i \neq j}(1+|i-j||\log \sigma|) \sigma^{|i-j|(1-\beta)} \quad \text { (by using Lemma 1.3) } \\
& \leq C(1+|\log \sigma|) \sigma^{1-2 \beta}=O\left(\sigma^{1-2 \beta}|\log \sigma|\right) .
\end{aligned}
$$

In (3.13) let us write $\varphi$ in the form

$$
\varphi=a W+\psi, \quad \text { where }\langle W, \psi\rangle=0, \text { and } a \in \mathbb{R} .
$$

Since $L(\psi)=L_{1}(\psi),(3.13)$ yields the following equality

$$
a\langle L(W), Z\rangle+\left\langle L_{1}(\psi), Z\right\rangle=\langle h, Z\rangle+\sum_{j=1}^{k} \gamma_{j}\left\langle W_{j}, Z\right\rangle
$$

From (3.24)-(3.26) it follows that

$$
\begin{aligned}
& \qquad\left|\left\langle L_{1}(\psi), Z\right\rangle\right|=\left|\left\langle\psi, L_{1}(Z)\right\rangle\right| \\
& \leq\left\{O\left(\sigma^{1-2 \beta}\right)+O\left(\sigma^{1-\frac{3 \beta}{2}}|\log \sigma|^{1 / 2}\right)+O\left(\sigma^{1-2 \beta}|\log \sigma|\right)\right\}\|\psi\|_{L_{2}} \\
& \leq O\left(\sigma^{1-2 \beta}|\log \sigma|\right)\|\varphi\|_{L^{2}}
\end{aligned}
$$

since $\|\psi\|_{L^{2}} \leq\|\varphi\|_{L^{2}}$. Then for $\varphi \in L_{\mu}^{\infty}$, by Lemma 1.5, we obtain

$$
\begin{aligned}
\left|\left\langle L_{1}(\psi), Z\right\rangle\right| & \leq O\left(\sigma^{1-2 \beta}|\log \theta|\right)\left(\int_{\mathbb{R}} e^{-2 \min _{1 \leq i \leq k}\left|x-\xi_{i}\right|} d x\right)^{1 / 2}\|\varphi\|_{\mu} \\
& \leq O\left(\sigma^{1-2 \beta}|\log \theta|\right)\left(\frac{1}{\mu}\left(k-(k-1) \sigma^{\mu}\right)^{1 / 2}\|\varphi\|_{\mu}\right. \\
& \leq O\left(\sigma^{1-\frac{5 \beta}{2}} \| \log \sigma \mid\right)\|\varphi\|_{\mu} .
\end{aligned}
$$


Next we estimate $\langle L(W), Z\rangle$ in (3.28). In fact, by using (1.7) we get

$$
\begin{aligned}
& \langle L(W), Z\rangle=\left\langle-W^{\prime \prime}+(1-2 W) W+\frac{2 W^{2}}{\omega}\langle W, W\rangle, Z\right\rangle \\
& =\left\langle-W^{2}+\frac{12 k}{\omega} W^{2}, Z\right\rangle-\sum_{\substack{i, j \\
i \neq j}}\left\langle U\left(x-\xi_{i}\right) U\left(x-\xi_{j}\right), Z\right\rangle \\
& +\left\langle\frac{2 W^{2}}{\omega} \sum_{\substack{i, j \\
i \neq j}}\left\langle U\left(x-\xi_{i}\right), U^{\prime}\left(x-\xi_{j}\right)\right\rangle, Z\right\rangle \\
& =\left(\frac{12 k}{\omega}-1\right)\left\langle W^{2}, \sum_{j=1}^{k}\left(x-\xi_{j}\right) U^{\prime}\left(x-\xi_{j}\right)+2 W\right\rangle+\sum_{i \neq j} \sigma^{|i-j|(1-\beta)}\|Z\|_{L^{1}} \\
& +O\left(\sigma^{1-\beta}|\log \sigma|\right)\left\|W^{2}\right\|_{L^{2}}\|Z\|_{L^{2} .}
\end{aligned}
$$

Note that

$$
\begin{gathered}
\left(\frac{12 k}{\omega}-1\right)\left\langle W^{2}, \sum_{j=1}^{k}\left(x-\xi_{j}\right) U^{\prime}\left(x-\xi_{j}\right)+2 W\right\rangle \\
=\left(\frac{12 k}{\omega}-1\right)\left\{\frac{5}{3} \int_{\mathbb{R}} W^{3}(x) d x\right. \\
\left.+\sum_{j=1}^{k} \sum_{\substack{i, \ell \\
\text { not both } j}} \int_{\mathbb{R}}\left(x-\xi_{j}\right) U\left(x-\xi_{i}\right) U\left(x-\xi_{\ell}\right) U^{\prime}\left(x-\xi_{j}\right) d x\right\}
\end{gathered}
$$

where the triple sum is estimated as follows,

$$
\begin{aligned}
& \left|\sum_{j=1}^{k} \sum_{\substack{i, \ell \\
\text { not both } j}} \int_{\mathbb{R}}\left(x-\xi_{j}\right) U^{\prime}\left(x-\xi_{j}\right) U\left(x-\xi_{i}\right) U\left(x-\xi_{\ell}\right) d x\right| \\
\leq & \left|\sum_{j=1}^{k} \sum_{\substack{i=j \\
\text { or } \ell=j}}^{k}\right|+\left|\sum_{j=1}^{k} \sum_{i=\ell \neq j} \ldots\right|+\left|\sum_{\substack{i=1 \\
j}}^{k} \sum_{\substack{i \neq j \\
\ell \neq j \\
i \neq \ell}} \ldots\right|=\Pi_{1}+\Pi_{2}+\Pi_{3},
\end{aligned}
$$


and

$$
\begin{aligned}
& \Pi_{1} \leq 2 \sum_{j=1}^{k} \sum_{|\ell-j| \geq 1}\left|\int_{\mathbb{R}}\left(x-\xi_{j}\right) U^{\prime}\left(x-\xi_{j}\right) U\left(x-\xi_{j}\right) U\left(x-\xi_{\ell}\right) d x\right| \\
& \leq 432 \sum_{j=1}^{k} \sum_{|\ell-j| \geq 1} \int_{\mathbb{R}}|x| e^{-2|x|} e^{-\left|x+\xi_{j}-\xi_{\ell}\right|} d x \\
& \leq 432 e^{-1} \sum_{j=1}^{k} \sum_{|\ell-j| \geq 1}(1+|\ell-j||\log \sigma|) \sigma^{|\ell-j|(1-\beta)} \\
& \leq C k|\log \sigma| \sigma^{1-\beta} \leq C \sigma^{1-2 \beta}|\log \sigma|, \\
& \Pi_{2} \leq 2 \sum_{j=1}^{k} \sum_{|\ell-j| \geq 1}\left|\int_{\mathbb{R}}\left(x-\xi_{j}\right) U^{\prime}\left(x-\xi_{j}\right) U^{2}\left(x-\xi_{\ell}\right) d x\right| \\
& \leq 432 \sum_{j=1}^{k} \sum_{|\ell-j| \geq 1} \int_{\mathbb{R}}|x| e^{-|x|} e^{-2\left|x+\xi_{j}-\xi_{\ell}\right|} d x \quad \text { (by (1.10)) } \\
& \leq 864 \sum_{j=1}^{k} \sum_{|\ell-j| \geq 1} e^{-\left|\xi_{j}-\xi_{\ell}\right|}\left(\left|\xi_{j}-\xi_{\ell}\right|+e^{-\left|\xi_{j}-\xi_{\ell}\right|}\right) \\
& \leq 864 \sum_{j=1}^{k} \sum_{|\ell-j| \geq 1} \sigma^{|i-\ell|(1-\beta)}\left(|i-\ell||\log \sigma|+\sigma^{|i-\ell|(1-\beta)}\right) \\
& \leq C k\left[\sigma^{1-\beta}|\log \sigma|+\sigma^{2(1-\beta)}\right]=O\left(\sigma^{1-2 \beta}|\log \sigma|\right), \\
& \Pi_{3} \leq \sum_{j=1}^{k} \sum_{\substack{\text { either }|i-\ell| \geq 2 \\
\text { or }|i-j| \geq 2 \\
\text { or }|\ell-j| \geq 2}}\left|\int_{\mathbb{R}}\left(x-\xi_{j}\right) U^{\prime}\left(x-\xi_{j}\right) U\left(x-\xi_{i}\right) U\left(x-\xi_{\ell}\right) d x\right| \\
& \leq 216 \sum_{j=1}^{k} \sum_{i, \ell \text { as above }} \int_{\mathbb{R}}|x| e^{-|x|} e^{-\left|x+\xi_{j}-\xi_{i}\right|} e^{-\left|x+\xi_{j}-\xi_{\ell}\right|} d x \\
& \leq 216 \sum_{j=1}^{k} \sum_{i, \ell \text { as above }} \begin{cases}\left.\sigma^{|i-\ell|(1-\beta)} \int_{\mathbb{R}}|x| e^{-|x|} d x \quad \text { (if }|i-\ell| \geq 2\right) \text {, or } \\
\sigma^{|i-j|(1-\beta)} \int_{\mathbb{R}}|x| e^{-\left|x+\xi_{j}-\xi_{\ell}\right|} d x & \text { (if }|i-j| \geq 2) \text {, or } \\
\sigma^{|\ell-j|(1-\beta)} \int_{\mathbb{R}}|x| e^{-\left|x+\xi_{j}-\xi_{i}\right|} d x & \text { (if }|\ell-j| \geq 2)\end{cases} \\
& \leq 216 k^{2} C|\log \sigma| \sigma^{2(1-\beta)} \leq C \sigma^{2(1-2 \beta)}|\log \sigma| .
\end{aligned}
$$

Therefore, we obtain

$$
\begin{gathered}
\left(\frac{12 k}{\omega}-1\right)\left\langle W^{2}, \sum_{j=1}^{k}\left(x-\xi_{j}\right) U^{\prime}\left(x-\xi_{j}\right)+2 W\right\rangle \\
\geq \frac{1}{2}\left\{\frac{5}{3} \int_{\mathbb{R}} W^{3}(x) d x+O\left(\sigma^{1-2 \beta}|\log \sigma|\right)\right\} \geq 6 k+O\left(\sigma^{1-2 \beta}|\log \sigma|\right),
\end{gathered}
$$

because

$$
\frac{12 k}{\omega}-1=\frac{12 k}{6 k+C \sigma^{1-2 \beta}|\log \sigma|}-1 \geq\left(2-\frac{1}{2}\right)-1=\frac{1}{2} \quad(\text { for } \sigma \text { small })
$$


and by Lemma 1.1,

$$
\int_{\mathbb{R}} W^{3}(x) d x>k \int_{\mathbb{R}} U^{3}(x) d x=\frac{36}{5} k .
$$

Moreover, we have

$$
\begin{gathered}
\|Z\|_{L^{1}} \leq\left\|\sum_{j=1}^{k}\left(x-\xi_{j}\right) U^{\prime}\left(x-\xi_{j}\right)\right\|_{L^{1}}+2\|W\|_{L^{1}} \\
=\|-W\|_{L^{1}}+2\|W\|_{L^{1}}=3\|W\|_{L^{1}}=18 k, \\
\|Z\|_{L^{2}} \leq\left\|\sum_{j=1}^{k}\left(x-\xi_{j}\right) U^{\prime}\left(x-\xi_{j}\right)\right\|_{L^{2}}+2\|W\|_{L^{2}} \\
\leq k\left(\int_{\mathbb{R}}|x|^{2}\left|U^{\prime}(x)\right|^{2} d x\right)^{1 / 2}+2 k \sqrt{6} \\
\leq k\left[6\left(\int_{\mathbb{R}}|x|^{2} e^{-2|x|}\right)^{1 / 2}+2 \sqrt{6}\right]=k\left(\frac{3}{2}+2 \sqrt{6}\right),
\end{gathered}
$$

and

$$
\left\|W^{2}\right\|_{L^{2}} \leq\|W\|_{L^{\infty}}\|W\|_{L^{2}} \leq \frac{12}{1-\sigma^{1-\beta}}\|W\|_{L^{2}} \leq 24 \sqrt{6} k, \text { for } \sigma \text { small. }
$$

Substituting these and (3.32) into (3.31), we obtain

$$
\begin{aligned}
\langle L(W), Z\rangle & \geq 6 k+O\left(\sigma^{1-2 \beta}|\log \sigma|\right)+O\left(\sigma^{1-2 \beta}\right) k+O\left(\sigma^{1-2 \beta}|\log \sigma|\right) k \\
& =k\left[6+O\left(\sigma^{1-2 \beta}|\log \sigma|\right)\right] .
\end{aligned}
$$

On the other hand, we have

$$
|\langle h, Z\rangle| \leq\|h\|_{\mu}\|Z\|_{L^{1}} \leq 18 k\|h\|_{\mu}
$$

and

$$
\left|\sum_{j=1}^{k} \gamma_{j}\left\langle W_{j}, Z\right\rangle\right| \leq\|\gamma\| \sum_{j=1}^{k}\left|\int_{\mathbb{R}} W_{j}(x) Z(x) d x\right|
$$

where

$$
\int_{\mathbb{R}} W_{j}(x) Z(x) d x=-\sum_{i(\neq j)=1}^{k} \int_{\mathbb{R}} U^{\prime}\left(x-\xi_{j}\right)\left[\left(x-\xi_{i}\right) U^{\prime}\left(x-\xi_{i}\right)+2 U\left(x-\xi_{i}\right)\right] d x
$$

because, when $i=j$, we have

$$
\begin{array}{r}
-\int_{\mathbb{R}} U^{\prime}\left(x-\xi_{j}\right)\left[\left(x-\xi_{j}\right) U^{\prime}\left(x-\xi_{j}\right)+2 U\left(x-\xi_{j}\right)\right] d x \\
=-\int_{\mathbb{R}}\left(x-\xi_{j}\right) U^{\prime}\left(x-\xi_{j}\right)^{2} d x=-\int_{\mathbb{R}} x\left|U^{\prime}(x)\right|^{2} d x=0 . \\
\text { (odd function) }
\end{array}
$$


Thus we get

$$
\begin{aligned}
&\left|\sum_{j=1}^{k} \gamma_{j}\left\langle W_{j}, Z\right\rangle\right| \leq\|\gamma\| \sum_{j=1}^{k} \sum_{i(\neq j)=1}^{k}\left[\int_{\mathbb{R}} 36|x| e^{-|x|} e^{\left|x+\xi_{i}-\xi_{j}\right|} d x\right. \\
&\left.\quad+\int_{\mathbb{R}} 72 e^{-|x|} e^{-\left|x+\xi_{i}-\xi_{j}\right|} d x\right] \\
& \leq\|\gamma\| \sum_{\substack{i, j \\
i \neq j}}\left[36\left(\int_{\mathbb{R}}|x| e^{-\epsilon_{0}|x|} d x\right) e^{-\left(1-\epsilon_{0}\right)\left|\xi_{i}-\xi_{j}\right|}\right. \\
&\left.+72(1+|i-j \| \log \sigma|) \sigma^{|i-j|(1-\beta)}\right] \\
& \leq C k\|\gamma\|\left[\sigma^{\left(1-\epsilon_{0}\right)(1-\beta)}+\sigma^{(1-\beta)}|\log \sigma|\right] \\
& \leq k\left(C_{1}\|h\|_{\mu}+C_{2}(\sigma)\|\varphi\|_{\mu}\right) O\left(\sigma^{\left(1-\epsilon_{0}\right)(1-\beta)}\right), \text { for any small } \epsilon_{0}>0,
\end{aligned}
$$

where we used (3.14) in Lemma 3.2.

LEMMA 3.3. Under the assumption that

$$
0<\beta<\frac{1}{2}
$$

it holds that

$$
\left|\frac{2}{\omega}\langle W, \varphi\rangle\right| \leq C_{3}\|h\|_{\mu}+C_{4}(\sigma)\|\varphi\|_{\mu},
$$

for a solution $(\varphi, \gamma)$ to the problem (3.13), where $C_{3}>0$ and $C_{4}(\sigma)>0$ are constants, with the property that $C_{4}(\sigma)=O\left(\sigma^{1-\frac{3 \beta}{2}}|\log \sigma|\right)$.

Proof. From (3.28), (3.30), (3.33), (3.34) and (3.35), we obtain

$$
\begin{aligned}
|a| \leq & \frac{1}{k\left[6+O\left(\sigma^{1-2 \beta}|\log \sigma|\right)\right]}\left\{O\left(\sigma^{1-\frac{5 \beta}{2}}|\log \sigma|\right)\|\varphi\|_{\mu}+18 k\|h\|_{\mu}\right. \\
& \left.+k\left(C_{1}\|h\|_{\mu}+C_{2}(\sigma)\|\varphi\|_{\mu}\right) O\left(\sigma^{\left(1-\epsilon_{0}\right)(1-\beta)}\right)\right\} \\
\leq & \frac{1}{6+O\left(\sigma^{1-2 \beta}|\log \sigma|\right)}\left\{\left(18+C_{1} O\left(\sigma^{\left(1-\epsilon_{0}\right)(1-\beta)}\right)\right)\|h\|_{\mu}\right. \\
& \left.+\left(O\left(\sigma^{1-\frac{3 \beta}{2}}|\log \sigma|\right)+C_{2}(\sigma) O\left(\sigma^{\left(1-\epsilon_{0}\right)(1-\beta)}\right)\right)\|\varphi\|_{\mu}\right\} \\
& \leq \hat{C}_{3}\|h\|_{\mu}+\hat{C}_{4}(\sigma)\|\varphi\|_{\mu},
\end{aligned}
$$

for $\sigma$ sufficiently small, where $\hat{C}_{3}>0$ is a uniform constant, and $C_{2}(\sigma)=O\left(\sigma^{1-\frac{3 \beta}{2}}\right)$ implies that

$$
\begin{aligned}
\hat{C}_{4}(\sigma) & =O\left(\sigma^{1-\frac{3 \beta}{2}}|\log \sigma|\right)+O\left(\sigma^{1-\frac{3 \beta}{2}+\left(1-\epsilon_{0}\right)(1-\beta)}\right) \\
& =O\left(\sigma^{1-\frac{3 \beta}{2}}|\log \sigma|\right)
\end{aligned}
$$

Since (3.27) implies that

$$
\langle W, \varphi\rangle=a\|W\|_{L^{2}}^{2}+\langle W, \psi\rangle=a\|W\|_{L^{2}}^{2}
$$


and we see that

$$
\begin{aligned}
\|W\|_{L^{2}}^{2} & =6 k+\sum_{\substack{i, j \\
i \neq j}} \int_{\mathbb{R}} U\left(x-\xi_{i}\right) U\left(x-\xi_{j}\right) d x \\
& \leq 6 k+\sum_{i \neq j} 36 \int_{\mathbb{R}} e^{-|x|} e^{-\left|x+\xi_{i}-\xi_{j}\right|} d x \quad(\text { by }(1.7)) \\
& \leq 6 k+\sum_{i \neq j} 36(1+|i-j||\log \sigma|) \sigma^{|i-j|(1-\beta) \quad \text { (by Lemma 1.3) }} \\
& \leq 6 k+C k \sigma^{1-\beta}|\log \sigma|=k\left(6+O\left(\sigma^{1-\beta}|\log \sigma|\right)\right) .
\end{aligned}
$$

Hence, we obtain

$$
\begin{gathered}
\left|\frac{2}{\omega}\langle W, \varphi\rangle\right| \leq \frac{2}{6 k}|\langle W, \varphi\rangle| \leq \frac{1}{3 k}|a|\|W\|_{L^{2}}^{2} \\
\leq \frac{1}{3 k}\left(\hat{C}_{3}\|h\|_{\mu}+\hat{C}_{4}(\sigma)\|\varphi\|_{\mu}\right) k\left(6+O\left(\sigma^{1-\beta}|\log \sigma|\right)\right) \\
=C_{3}\|h\|+C_{4}(\sigma)\|\varphi\|_{\mu},
\end{gathered}
$$

where

$$
\begin{gathered}
C_{3}\left(\geq \hat{C}_{3}\left(2+\frac{1}{3} O\left(\sigma^{1-\beta}|\log \sigma|\right)\right)\right) \text { is a constant, and } \\
C_{4}(\sigma)=\hat{C}_{4}(\sigma)\left(2+\frac{1}{3} O\left(\sigma^{1-\beta}|\log \sigma|\right)\right)=O\left(\sigma^{1-\frac{3 \beta}{2}}|\log \sigma|\right) .
\end{gathered}
$$

Thus the lemma is proved.

With all these preparations, we are going to conduct an a priori estimate of the solution $\varphi$ of the problem (3.13), if it exists. According to (3.1), (2.1) and (2.2), a solution $\varphi$ to Eq. (3.13) satisfies the integral equation

$$
\varphi(x)=\frac{1}{2} \int_{\mathbb{R}} e^{-|x-y|}\left(2 W \varphi-\frac{2 W^{2}}{\omega}\langle W, \varphi\rangle+h+\gamma \cdot \nabla_{\xi} W\right) d y .
$$

In order to get a sharp estimate of the integral term involving $2 W \varphi$ on the righthand side of (3.43), we use the cut-off decomposition of $\varphi$ introduced in (1.22). Recall that by (1.23),

$$
\begin{gathered}
\Omega=\Omega_{0} \cup \Omega_{1} \cup \cdots \cup \Omega_{k}, \quad \text { (mutually disjoint) } \\
\Omega_{0}=\mathbb{R} \backslash \bigcup_{j=1}^{k} \Omega_{j} \text { and } \Omega_{j}=\left[\xi_{j}-x_{\sigma}, \xi_{j}+x_{\sigma}\right], \quad j=1, \ldots, k, \\
\text { where } x_{\sigma}=r_{\sigma} / 4=\frac{1}{4}(1-\beta)|\log \sigma|, \text { so that } \\
e^{-x_{\sigma}}=e^{-r_{\sigma} / 4} \leq \sigma^{\frac{1}{4}(1-\beta)} .
\end{gathered}
$$

For any $j=0,1, \ldots, k$, we shall consider the integral terms

$$
\begin{gathered}
\frac{1}{2} \int_{\Omega_{j}} e^{-|x-y|}\left[2 W(y) \sum_{\ell=0}^{k} \varphi_{\ell}(y)-\frac{2 W^{2}}{\omega}\langle W, \varphi\rangle+h+\gamma \cdot \nabla_{\xi} W\right] d y \\
=J_{j}^{1}+J_{j}^{2}+J_{j}^{3}+\frac{1}{2} \int_{\Omega_{j}} e^{-|x-y|} h(y) d y .
\end{gathered}
$$


Since we choose $0<\mu \leq 1 / 3$, we have $\frac{1}{1-\mu} \leq \frac{3}{2}$. For the simplicity of notation, we set

$$
\rho(x)=\rho(x, \xi) \triangleq \min _{1 \leq i \leq k}\left|x-\xi_{i}\right|, \quad x \in \mathbb{R} .
$$

First of all, it is easy to see that

$$
\begin{gathered}
\left|\frac{1}{2} \int_{\mathbb{R}} e^{-|x-y|} h(y) d y\right| \leq \frac{1}{2} \int_{\mathbb{R}} e^{-|x-y|} e^{-\mu \rho(y)}\|h\|_{\mu} d y \\
=\frac{1}{2} \int_{\mathbb{R}} e^{-(1-\mu)|x-y|} e^{-\mu\left(|x-y|+\min _{1 \leq i \leq k}\left|y-\xi_{i}\right|\right)} d y\|h\|_{\mu} \\
\leq \frac{1}{2} e^{-\mu \min _{1 \leq i \leq k}\left|x-\xi_{i}\right|}\left(\int_{\mathbb{R}} e^{-(1-\mu)|x-y|} d y\right)\|h\|_{\mu} \\
=\frac{1}{1-\mu} e^{-\mu \rho(x)}\|h\|_{\mu} .
\end{gathered}
$$

In $(3.44)$,

$$
\begin{aligned}
& J_{j}^{1}=\frac{1}{2} \int_{\Omega_{j}} e^{-|x-y|} 2 W(y) \sum_{\ell=0}^{k} \varphi_{\ell}(y) d y \\
& J_{j}^{2}=\frac{1}{2} \int_{\Omega_{j}} e^{-|x-y|}\left(-\frac{2 W^{2}(y)}{\omega}\langle W, \varphi\rangle\right) d y, \text { and } \\
& J_{j}^{3}=\frac{1}{2} \int_{\Omega_{j}} e^{-|x-y|} \gamma \cdot \nabla_{\xi} W(y) d y .
\end{aligned}
$$

We start with the estimate of $J_{j}^{1}$. Then $J^{1}=\sum_{j=0}^{k} J_{j}^{1}$. Note that for any $y \in \Omega_{0}$,

$$
\min _{1 \leq i \leq k}\left|y-\xi_{i}\right| \geq x_{\sigma}
$$

We find that

$$
\begin{aligned}
& \left|J_{0}^{1}\right|=\left|\int_{\Omega_{0}} e^{-|x-y|} W(y) \varphi(y) d y\right| \leq \int_{\Omega_{0}} e^{-|x-y|}|W(y)||\varphi(y)| d y \\
& \quad \leq \int_{\Omega_{0}} e^{-|x-y|}\left(6 \sum_{j=1}^{k} e^{-\left|y-\xi_{j}\right|}\right)\left(e^{-\mu \min _{1 \leq i \leq k}\left|y-\xi_{i}\right|}\|\varphi\|_{\mu}\right) d y \\
& \leq 6 \int_{\Omega_{0}} e^{-|x-y|}\left(\sum_{j=1}^{k} e^{-(1-\mu)\left|y-\xi_{j}\right|}\right)\left(e^{-2 \mu \min _{1 \leq i \leq k}\left|y-\xi_{i}\right|}\right) d y\left\|_{\varphi}\right\|_{\mu} \\
& \quad \leq 6 e^{-2 \mu \rho(x)} \int_{\Omega_{0}} e^{-(1-2 \mu)|x-y|}\left(\sum_{j=1}^{k} e^{-(1-\mu)\left|y-\xi_{j}\right|}\right) d y\|\varphi\|_{\mu},
\end{aligned}
$$

because

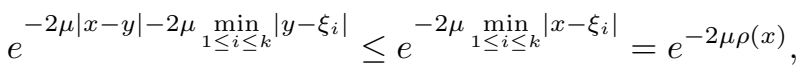

where $|x-y|+\min _{1 \leq i \leq k}\left|y-\xi_{i}\right|=\min _{1 \leq i \leq k}\left\{|x-y|+\left|y-\xi_{i}\right|\right\} \geq \min _{1 \leq i \leq k}\left|x-\xi_{i}\right|$. 
Moreover,

$$
\begin{aligned}
& \sum_{j=1}^{k} e^{-(1-\mu)\left|y-\xi_{j}\right|}=e^{-(1-\mu)\left|y-\xi_{j_{0}}\right|}+\sum_{j \neq j_{0}} e^{-(1-\mu)\left|y-\xi_{j}\right|} \\
& \quad\left(\text { where }\left|y-\xi_{j_{0}}\right|=\min _{1 \leq j \leq k}\left|y-\xi_{j}\right|\right) \\
& \leq e^{-(1-\mu) x_{\sigma}}+2 e^{-(1-\mu)\left(x_{\sigma}+r_{\sigma}\right)}+2 e^{-(1-\mu)\left(x_{\sigma}+2 r_{\sigma}\right)}+\cdots \\
& \leq \frac{2 e^{-(1-\mu) x_{\sigma}}}{1-e^{-(1-\mu) r_{\sigma}}}=\frac{2 \sigma^{(1-\mu)(1-\beta) / 4}}{1-\sigma^{(1-\mu)(1-\beta)}} \leq 4 \sigma^{(1-\mu)(1-\beta) / 4},
\end{aligned}
$$

for $\sigma$ small. Therefore, we get

$$
\begin{aligned}
\left|J_{0}^{1}\right| & \leq 6 e^{-2 \mu \rho(x)}\left(\int_{\Omega_{0}} e^{-(1-2 \mu)|x-y|} d y\right) 4 \sigma^{(1-\mu)(1-\beta) / 4}\|\varphi\|_{\mu} \\
& =\frac{48}{1-2 \mu} \sigma^{(1-\mu)(1-\beta) / 4} e^{-2 \mu \rho(x)}\|\varphi\|_{\mu} \\
& \leq 144 \sigma^{(1-\mu)(1-\beta) / 4} e^{-2 \mu \rho(x)}\|\varphi\|_{\mu} .
\end{aligned}
$$

For $j=1, \ldots, k$, due to $\operatorname{supp} \theta\left(\cdot-\xi_{\ell}\right) \cap \Omega_{j}=\emptyset$ for $\ell \neq j$ and $\left|\theta\left(y-\xi_{j}\right)\right| \leq 1$ for $y \in \mathbb{R}$, we have

$$
\begin{aligned}
& \left|J_{j}^{1}\right|=\left|\int_{\Omega_{j}} e^{-|x-y|} W(y) \sum_{\ell=0}^{k} \varphi_{\ell}(y) d y\right| \\
& =\left|\int_{\Omega_{j}} e^{-|x-y|} W(y) \theta\left(y-\xi_{j}\right) \varphi(y) d y\right| \\
& \leq \sum_{m=1}^{k} \int_{\Omega_{j}} e^{-|x-y|} U\left(y-\xi_{m}\right)|\varphi(y)| d y \\
& =\sum_{m=1}^{k} \int_{\xi_{j}-x_{\sigma}}^{\xi_{j}+x_{\sigma}} e^{-|x-y|} U\left(y-\xi_{m}\right)|\varphi(y)| d y \\
& =\sum_{m=1}^{k} \int_{-x_{\sigma}}^{x_{\sigma}} e^{-\left|x-y-\xi_{j}\right|} U\left(y+\xi_{j}-\xi_{m}\right)\left|\varphi\left(y+\xi_{j}\right)\right| d y \\
& \leq 6 \sum_{m=1}^{k} \int_{-x_{\sigma}}^{x_{\sigma}} e^{-\left|x-y-\xi_{j}\right|} e^{-\left|y+\xi_{j}-\xi_{m}\right|} e^{-\mu \min _{1 \leq i \leq k}\left|y+\xi_{j}-\xi_{i}\right|} d y\|\varphi\|_{\mu} \\
& \leq 6 \sum_{m=1}^{k} \int_{-x_{\sigma}}^{x_{\sigma}} e^{-\left|x-y-\xi_{j}\right|} e^{-(1-\mu)\left|y+\xi_{j}-\xi_{m}\right|} e^{-2 \mu \min _{1 \leq i \leq k}\left|y+\xi_{j}-\xi_{i}\right|} d y\|\varphi\|_{\mu} \\
& \leq 6 \sum_{m=1}^{k} \int_{-x_{\sigma}}^{x_{\sigma}} e^{-(1-2 \mu)\left|x-y-\xi_{j}\right|} e^{-(1-\mu)\left|y+\xi_{j}-\xi_{m}\right|} e^{-2 \mu \min _{1 \leq i \leq k}\left|x-\xi_{i}\right|} d y\|\varphi\|_{\mu} \\
& =6 e^{-2 \mu \rho(x)}\left(\int_{-x_{\sigma}}^{x_{\sigma}} e^{-(1-2 \mu)\left|x-y-\xi_{j}\right|} e^{-(1-\mu)|y|} d y\right)\|\varphi\|_{\mu} \\
& +6 e^{-2 \mu \rho(x)}\left(\sum_{m \neq j} \int_{-x_{\sigma}}^{x_{\sigma}} e^{-(1-2 \mu)\left|x-y-\xi_{j}\right|} e^{-(1-\mu)\left|y+\xi_{j}-\xi_{m}\right|} d y\right)\|\varphi\|_{\mu}
\end{aligned}
$$


where, for $m \neq j$, we have

$$
\sum_{m \neq j} e^{-(1-\mu)\left|y+\xi_{j}-\xi_{m}\right|} \leq \frac{2 \sigma^{\frac{3}{4}(1-\mu)(1-\beta)}}{1-\sigma^{1-\beta}} \leq \frac{2 \sqrt{2}}{\sqrt{2}-1} \sigma^{\frac{3}{4}(1-\mu)(1-\beta)} .
$$

With the key observation that for any $x \in \mathbb{R}, y \in\left[-x_{\sigma}, x_{\sigma}\right]$, there is an index $j_{0}=j_{0}(x, y) \in\{1, \ldots, k\}$, such that

$$
\left|x-y-\xi_{j_{0}}\right|=\min _{1 \leq j \leq k}\left|x-y-\xi_{j}\right|,
$$

we can calculate

$$
\begin{aligned}
& \sum_{j=1}^{k} e^{-(1-2 \mu)\left|x-y-\xi_{j}\right|}=e^{-(1-2 \mu)\left|x-y-\xi_{j_{0}}\right|}+\sum_{j \neq j_{0}} e^{-(1-2 \mu)\left|x-y-\xi_{j}\right|} \\
& \leq 1+e^{-(1-2 \mu)\left|x-y-\xi_{j_{0}-1}\right|}+e^{-(1-2 \mu)\left|x-y-\xi_{j_{0}+1}\right|}+\cdots \\
& \leq 1+2\left(e^{-(1-2 \mu) r_{\sigma} / 2}+e^{-(1-2 \mu) 3 r_{\sigma} / 2}+e^{-(1-2 \mu) 5 r_{\sigma} / 2}+\cdots\right) \\
& \quad=1+\frac{2 e^{-(1-2 \mu) r_{\sigma} / 2}}{1-e^{-(1-2 \mu) r_{\sigma}}}=1+\frac{2 \sigma^{\frac{1}{2}(1-2 \mu)(1-\beta)}}{1-\sigma^{(1-2 \mu)(1-\beta)}}<C_{5},
\end{aligned}
$$

where $C_{5}>0$ is a constant independent of small $\sigma$. By summing up $\left|J_{j}^{1}\right|$ for all $j=1, \ldots, k$, and using (3.50) and (3.51), we get

$$
\begin{aligned}
& \left|J^{1}\right|=\left|\sum_{j=0}^{k} J_{j}^{1}\right| \leq \sum_{j=0}^{k}\left|J_{j}^{1}\right| \leq e^{-2 \mu \rho(x)}\|\varphi\|_{\mu}\left\{144 \sigma^{(1-\mu)(1-\beta) / 4}\right. \\
& +\int_{-x_{\sigma}}^{x_{\sigma}} 6 \sum_{j=1}^{k} e^{-(1-2 \mu)\left|x-y-\xi_{j}\right|} e^{-(1-\mu)|y|} d y \\
& \left.+\sum_{m \neq j} \int_{-x_{\sigma}}^{x_{\sigma}} 6 \sum_{j=1}^{k} e^{-(1-2 \mu)\left|x-y-\xi_{j}\right|} \sigma^{\frac{3}{4}(1-\mu)(1-\beta)} d y\right\} \\
& \leq e^{-2 \mu \rho(x)}\|\varphi\|_{\mu}\left\{144 \sigma^{1 / 12}+6 C_{5} \frac{2}{1-\mu}+6 C_{5} \int_{-x_{\sigma}}^{x_{\sigma}} \sum_{m(\neq j)=1}^{k} e^{-(1-\mu)\left|y+\xi_{j}-\xi_{m}\right|} d y\right\} \\
& \leq e^{-2 \mu \rho(x)}\|\varphi\|_{\mu}\left\{144 \sigma^{1 / 12}+18 C_{5}+6 C_{5} \frac{2 \sqrt{2}}{\sqrt{2}-1} \sigma^{\frac{3}{4}(1-\mu)(1-\beta)} 2 x_{\sigma}\right\} \\
& \leq e^{-2 \mu \rho(x)}\|\varphi\|_{\mu}\left\{18 C_{5}+144 \sigma^{1 / 12}+15 \sqrt{2} C_{5} \sigma^{\frac{3}{4}(1-\mu)(1-\beta)}\left(C_{0}+|\log \sigma|\right)\right\},
\end{aligned}
$$

where we computed

$$
\frac{2 \sqrt{2}}{\sqrt{2}-1}<\frac{2 \sqrt{2}}{0.4}=5 \sqrt{2}, \text { and } 2 x_{\sigma}=\frac{r_{\sigma}}{2} \leq \frac{1}{2}(1-\beta)|\log \sigma| .
$$

Secondly we estimate $J_{j}^{2}$ and $J^{2}=\sum_{j=0}^{k} J_{j}^{2}$. With similar observations as in the processing of $J^{1}$, by using Lemma 3.3 and (3.47) and (3.51), we can get the following 
estimates. First,

$$
\begin{gathered}
\left|J_{0}^{2}\right|=\left|\frac{1}{2} \int_{\Omega_{0}} e^{-|x-y|}\left(-\frac{2 W^{2}(y)}{\omega}\langle W, \varphi\rangle\right) d y\right| \\
\leq \frac{1}{2}\left(C_{3}\|h\|_{\mu}+C_{4}(\sigma)\|\varphi\|_{\mu}\right) \int_{\Omega_{0}} e^{-|x-y|} W^{2}(y) d y \\
\leq 18\left(C_{3}\|h\|_{\mu}+C_{4}(\sigma)\|\varphi\|_{\mu}\right) \int_{\Omega_{0}} e^{-|x-y|}\left(\sum_{j=1}^{k} e^{-\left|y-\xi_{j}\right|}\right)^{2} d y \\
\leq 18\left(C_{3}\|h\|_{\mu}+C_{4}(\sigma)\|\varphi\|_{\mu}\right) \int_{\Omega_{0}} e^{-|x-y|}\left(\sum_{j=1}^{k} e^{-(1-\mu)\left|y-\xi_{j}\right|}\right)^{-2 \mu \min _{1 \leq k \leq k}\left|y-\xi_{i}\right|} d y \\
\leq 18 e^{-2 \mu \rho(x)}\left(C_{3}\|h\|_{\mu}+C_{4}(\sigma)\|\varphi\|_{\mu}\right) \int_{\Omega_{0}} e^{-(1-2 \mu)|x-y|} 16 \sigma^{(1-\mu)(1-\beta) / 2} d y \\
=576 e^{-2 \mu \rho(x)} \frac{\sigma^{(1-\mu)(1-\beta) / 2}}{1-2 \mu}\left(C_{3}\|h\|_{\mu}+C_{4}(\sigma)\|\varphi\|_{\mu}\right) \\
=e^{-2 \mu \rho(x)}\left(C_{6}(\sigma)\|h\|_{\mu}+C_{7}(\sigma)\|\varphi\|_{\mu}\right),
\end{gathered}
$$

where

$$
\begin{gathered}
C_{6}(\sigma)=C_{3} \frac{576}{1-2 \mu} \sigma^{(1-\mu)(1-\beta) / 2}=O\left(\sigma^{(1-\mu)(1-\beta) / 2}\right) \\
C_{7}(\sigma)=C_{4}(\sigma) O\left(\sigma^{(1-\mu)(1-\beta) / 2}\right)=O\left(\sigma^{1-\frac{3 \beta}{2}+(1-\mu)(1-\beta) / 2}|\log \sigma|\right) \\
=O\left(\sigma^{1-\frac{3 \beta}{2}}\right),
\end{gathered}
$$

where $(1-\mu)(1-\beta) / 2 \geq \frac{2}{3} \cdot \frac{1}{2} \cdot \frac{1}{2}=1 / 6$. Next, for $j=1, \ldots, k$, we have

$$
\begin{gathered}
\left|J_{j}^{2}\right|=\left|\frac{1}{2} \int_{\Omega_{j}} e^{-|x-y|}\left(-\frac{2 W^{2}(y)}{\omega}\langle W, \varphi\rangle\right) d y\right| \\
\leq \frac{1}{2}\left(C_{3}\|h\|_{\mu}+C_{4}(\sigma)\|\varphi\|_{\mu}\right)\left\{\int_{\Omega_{j}} e^{-|x-y|} \sum_{\ell=1}^{k} U^{2}\left(y-\xi_{\ell}\right) d y\right. \\
\left.+\int_{\Omega_{j}} e^{-|x-y|} \sum_{m, \ell} U\left(y-\xi_{i}\right) U\left(y-\xi_{\ell}\right) d y\right\} \\
\leq 18\left(C_{3}\|h\|_{\mu}+C_{4}(\sigma)\|\varphi\|_{\mu}\right)\left\{\sum_{\ell=1}^{k} \int_{-x_{\sigma}}^{x_{\sigma}} e^{-\left|x-y-\xi_{j}\right|} e^{-2\left|y+\xi_{j}-\xi_{\ell}\right|} d y\right. \\
\left.+\sum_{\substack{m, \ell \\
m \neq \ell}} \int_{-x_{\sigma}}^{x_{\sigma}} e^{-\left|x-y-\xi_{j}\right|} e^{-\left|y+\xi_{j}-\xi_{m}\right|} e^{-\left|y+\xi_{j}-\xi_{\ell}\right|} d y\right\}
\end{gathered}
$$




$$
\begin{aligned}
\leq 18\left(C_{3}\|h\|_{\mu}+\right. & \left.C_{4}(\sigma)\|\varphi\|_{\mu}\right) e^{-2 \mu \min _{1 \leq i \leq k}\left|x-\xi_{i}\right|}\left\{\int_{-x_{\sigma}}^{x_{\sigma}} e^{-(1-2 \mu)\left|x-y-\xi_{j}\right|} e^{-2(1-\mu)|y|} d y\right. \\
& +\sum_{\ell(\neq j)=1}^{k} \int_{-x_{\sigma}}^{x_{\sigma}} e^{-(1-2 \mu)\left|x-y-\xi_{j}\right|} e^{-2(1-\mu)\left|y+\xi_{j}-\xi_{\ell}\right|} d y \\
& \left.+\sum_{\substack{m, \ell \\
m \neq \ell}} \int_{-x_{\sigma}}^{x_{\sigma}} e^{-(1-2 \mu)\left|x-y-\xi_{j}\right|} e^{-(1-\mu)\left|y+\xi_{j}-\xi_{m}\right|} e^{-(1-\mu)\left|y+\xi_{j}-\xi_{\ell}\right|} d y\right\} .
\end{aligned}
$$

Summing up (3.53) and (3.55) for $j=1, \ldots, k$, we obtain

$$
\begin{gathered}
\left|J^{2}\right| \leq \sum_{j=0}^{k}\left|J_{j}^{2}\right| \leq e^{-2 \mu \rho(x)}\left\{C_{6}(\sigma)\|h\|_{\mu}+C_{7}(\sigma)\|\varphi\|_{\mu}\right. \\
+18\left(C_{3}\|h\|_{\mu}+C_{4}(\sigma)\|\varphi\|_{\mu}\right)\left[\int_{-x_{\sigma}}^{x_{\sigma}} \sum_{j=1}^{k} e^{-(1-2 \mu)\left|x-y-\xi_{j}\right|} e^{-2(1-\mu)|y|} d y\right. \\
+\int_{-x_{\sigma}}^{x_{\sigma}} \sum_{j=1}^{k} e^{-(1-2 \mu)\left|x-y-\xi_{j}\right|}\left(\sum_{\ell(\neq j)=1}^{k} e^{-2(1-\mu)\left|y+\xi_{j}-\xi_{\ell}\right|}\right) d y \\
\left.+\int_{-x_{\sigma}}^{x_{\sigma}} \sum_{j=1}^{k} e^{-(1-2 \mu)\left|x-y-\xi_{j}\right|}\left(\sum_{\substack{m, \ell \\
m \neq \ell}} e^{-(1-\mu)\left|y+\xi_{j}-\xi_{m}\right|} e^{-(1-\mu)\left|y+\xi_{j}-\xi_{\ell}\right|}\right) d y\right]
\end{gathered}
$$

where

$$
\begin{gathered}
\sum_{j=1}^{k} e^{-(1-2 \mu)\left|x-y-\xi_{j}\right|} \leq C_{5}, \quad \text { by }(3.51), \\
\sum_{\ell(\neq j)=1} e^{-2(1-\mu)\left|y+\xi_{j}-\xi_{\ell}\right|} \leq e^{-2(1-\mu) \frac{3}{4} r_{\sigma}}\left[1+2 e^{-2(1-\mu) r_{\sigma}}+\cdots\right] \\
\leq \frac{2 \sigma^{\frac{3}{2}(1-\mu)(1-\beta)}}{1-\sigma^{2(1-\mu)(1-\beta)}} \leq C \sigma^{\frac{3}{2}(1-\mu)(1-\beta)}, \text { for all } y \in\left[-x_{\sigma}, x_{\sigma}\right]
\end{gathered}
$$

and

$$
\begin{gathered}
\sum_{\substack{m, \ell \\
m \neq \ell}} e^{-(1-\mu)\left|y+\xi_{j}-\xi_{m}\right|} e^{-(1-\mu)\left|y+\xi_{j}-\xi_{\ell}\right|} \leq \sum_{\substack{m, \ell \\
m \neq \ell}} e^{-(1-\mu)\left|\xi_{m}-\xi_{\ell}\right|} \\
\leq \sum_{m=1}^{k} \sum_{\ell \neq m} \sigma^{|m-\ell|(1-\mu)(1-\beta)} \\
\leq k \frac{2 \sigma^{(1-\mu)(1-\beta)}}{1-\sigma^{(1-\mu)(1-\beta)}} \leq C \sigma^{(1-\mu)(1-\beta)-\beta}, \quad \forall y \in\left[-x_{\sigma}, x_{\sigma}\right] .
\end{gathered}
$$


Therefore, we come up with

$$
\begin{gathered}
\left|J^{2}\right| \leq e^{-2 \mu \rho(x)}\left\{C_{6}(\sigma)\|h\|_{\mu}+C_{7}(\sigma)\|\varphi\|_{\mu}\right. \\
+18\left(C_{3}\|h\|_{\mu}+C_{4}(\sigma)\|\varphi\|_{\mu}\right) C_{5}\left[\frac{1}{1-\mu} \sigma^{\frac{1}{2}(1-\mu)(1-\beta)}\right. \\
\left.\left.+C \sigma^{\frac{3}{2}(1-\mu)(1-\beta)}\left(C_{0}+|\log \sigma|\right)+C \sigma^{(1-\mu)(1-\beta)-\beta}\left(C_{0}+|\log \sigma|\right)\right]\right\} \\
\leq e^{-2 \mu \rho(x)}\left[C_{8}(\sigma)\|h\|_{\mu}+C_{9}(\sigma)\|\varphi\|_{\mu}\right]
\end{gathered}
$$

where

$$
\begin{aligned}
C_{8}(\sigma)=C_{6}(\sigma)+18 C_{3} C_{5}[\cdots]= & O\left(\sigma^{\frac{1}{2}(1-\mu)(1-\beta)}\right)+O\left(\sigma^{(1-\mu)(1-\beta)-\beta}|\log \sigma|\right) \\
C_{9}(\sigma)=C_{7}(\sigma)+18 C_{4}(\sigma) C_{5}[\cdots] & =O\left(\sigma^{1-\frac{3 \beta}{2}}\right)+O\left(\sigma^{1-\frac{3 \beta}{2}} \sigma^{(1-\mu)(1-\beta)-\beta}|\log \sigma|^{2}\right) \\
& =O\left(\sigma^{1-\frac{3 \beta}{2}}\right),
\end{aligned}
$$

provided that

$$
(1-\mu)(1-\beta)-\beta=1-\mu-(2-\mu) \beta>0, \quad \text { or } \beta<\frac{1-\mu}{2-\mu} .
$$

When we choose $\mu=1 / 3$, then (3.58) implies $\beta<\frac{2}{5}$. For $\mu$ close to $0, \beta$ is close to $1 / 2$.

Thirdly we estimate $J_{j}^{3}$ and $J^{3}=\sum_{j=0}^{k} J_{j}^{3}$, with the aid of Lemma 3.2. In fact,

$$
\begin{aligned}
\left|J_{0}^{3}\right| & =\left|\frac{1}{2} \int_{\Omega_{0}} e^{-|x-y|} \gamma \cdot \nabla_{\xi} W(y) d y\right|=\left|\frac{1}{2} \int_{\Omega_{0}} e^{-|x-y|} \sum_{j=1}^{k} \gamma_{j} W_{j}(y) d y\right| \\
& \leq \frac{1}{2}\|\gamma\| \sum_{j=1}^{k} \int_{\Omega_{0}} e^{-|x-y|} 6 e^{-\left|y-\xi_{j}\right|} d y \\
& \leq 3\|\gamma\| \sum_{j=1}^{k} \int_{\Omega_{0}} e^{-(1-\mu)\left(|x-y|+\left|y-\xi_{j}\right|\right)} d y e^{-\mu \min _{1 \leq i \leq k}\left|x-\xi_{i}\right|} \\
& \leq 3\left(C_{1}\|h\|_{\mu}+C_{2}(\sigma)\|\varphi\|_{\mu}\right) e^{-\mu \rho(x)} \sum_{j=1}^{k} \int_{\Omega_{0}} e^{-(1-\mu)\left(|x-y|+\left|y-\xi_{j}\right|\right)} d y,
\end{aligned}
$$

Where, by (3.47a) and (3.47b), we have

$$
\sum_{j=1}^{k} e^{-(1-\mu)\left|y-\xi_{j}\right|} \leq 4 \sigma^{(1-\mu)(1-\beta) / 4},
$$

so that

$$
\begin{aligned}
\left|J_{0}^{3}\right| & \leq 3\left(C_{1}\|h\|_{\mu}+C_{2}(\sigma)\|\varphi\|_{\mu}\right) e^{-\mu \rho(x)}\left(4 \sigma^{(1-\mu)(1-\beta) / 4}\right) \int_{\Omega_{0}} e^{-(1-\mu)|x-y|} d y \\
& \leq \frac{24}{1-\mu} e^{-\mu \rho(x)}\left(C_{1}\|h\|_{\mu}+C_{2}(\sigma)\|\varphi\|_{\mu}\right) \sigma^{\frac{1}{4}(1-\mu)(1-\beta)} .
\end{aligned}
$$


For $j=1, \ldots, k$, we have

$$
\begin{aligned}
&\left|J_{j}^{3}\right|=\left|\frac{1}{2} \int_{\Omega_{j}} e^{-|x-y|} \sum_{i=1}^{k} \gamma_{i} W_{i}(y) d y\right| \leq \frac{1}{2}\|\gamma\| \sum_{i=1}^{k} \int_{\Omega_{j}} e^{-|x-y|}\left|U^{\prime}\left(y-\xi_{i}\right)\right| d y \\
& \leq 3\left(C_{1}\|h\|_{\mu}+C_{2}(\sigma)\|\varphi\|_{\mu}\right) e^{-\mu \rho(x)} \sum_{i=1}^{k} \int_{\Omega_{j}} e^{-(1-\mu)\left(|x-y|+\left|y-\xi_{i}\right|\right)} d y \\
&=3\left(C_{1}\|h\|_{\mu}+C_{2}(\sigma)\|\varphi\|_{\mu}\right) e^{-\mu \rho(x)} \sum_{i=1}^{k} \int_{-x_{\sigma}}^{x_{\sigma}} e^{-(1-\mu)\left(\left|x-y-\xi_{j}\right|+\left|y+\xi_{j}-\xi_{i}\right|\right)} d y \\
& \leq 3\left(C_{1}\|h\|_{\mu}+C_{2}(\sigma)\|\varphi\|_{\mu}\right) e^{-\mu \rho(x)}\left[\int_{-x_{\sigma}}^{x_{\sigma}} e^{-(1-\mu)\left|x-y-\xi_{j}\right|} e^{-(1-\mu)|y|} d y\right. \\
&\left.+\int_{-x_{\sigma}}^{x_{\sigma}} e^{-(1-\mu)\left|x-y-\xi_{j}\right|} \sum_{i(\neq j)=1}^{k} e^{-(1-\mu)\left|y+\xi_{j}-\xi_{i}\right|} d y\right] \\
& \leq 3\left(C_{1}\|h\|_{\mu}+C_{2}(\sigma)\|\varphi\|_{\mu}\right) e^{-\mu \rho(x)}\left[\int_{-x_{\sigma}}^{x_{\sigma}} e^{-(1-\mu)\left|x-y-\xi_{j}\right|} e^{-(1-\mu)|y|} d y\right. \\
&\left.+\int_{-x_{\sigma}}^{x_{\sigma}} e^{-(1-\mu)\left|x-y-\xi_{j}\right|} \cdot \frac{2 \sigma^{\frac{3}{4}(1-\mu)(1-\beta)}}{1-\sigma^{(1-\mu)(1-\beta)}} d y\right] .
\end{aligned}
$$

Summing up (3.59) and (3.60) for $j=1, \ldots, k$, by (3.51), we obtain

$$
\begin{gathered}
\left|J^{3}\right| \leq e^{-\mu \rho(x)}\left(C_{1}\|h\|_{\mu}+C_{2}(\sigma)\|\varphi\|_{\mu}\right)\left\{\frac{24}{1-\mu} \sigma^{\frac{1}{4}(1-\mu)(1-\beta)}\right. \\
\left.+3 C_{5}\left[\int_{-x_{\sigma}}^{x_{\sigma}} e^{-(1-\mu)|y|} d y+\int_{-x_{\sigma}}^{x_{\sigma}} 4 \sigma^{\frac{3}{4}(1-\mu)(1-\beta)} d y\right]\right\} \quad(\text { for } \sigma \text { small }) \\
\leq e^{-\mu \rho(x)}\left(C_{1}\|h\|_{\mu}+C_{2}(\sigma)\|\varphi\|_{\mu}\right)\left\{\frac{24}{1-\mu} \sigma^{\frac{1}{4}(1-\mu)(1-\beta)}\right. \\
\left.+3 C_{5}\left[\frac{2}{1-\mu} \sigma^{\frac{1}{4}(1-\mu)(1-\beta)}+2 \sigma^{\frac{3}{4}(1-\mu)(1-\beta)}\left(C_{0}+|\log \sigma|\right)\right]\right\} \\
=e^{-\mu \rho(x)}\left(C_{10}(\sigma)\|h\|_{\mu}+C_{11}(\sigma)\|\varphi\|_{\mu}\right),
\end{gathered}
$$

where

$$
\begin{aligned}
& C_{10}(\sigma)=O\left(\sigma^{\frac{1}{4}(1-\mu)(1-\beta)}\right), \text { and } \\
& C_{11}(\sigma)=C_{2}(\sigma) O\left(\sigma^{\frac{1}{4}(1-\mu)(1-\beta)}\right)=O\left(\sigma^{1-\frac{3 \beta}{2}+\frac{1}{4}(1-\mu)(1-\beta)}\right) .
\end{aligned}
$$

We summarize these estimates in the following lemma.

LEMMA 3.4. Under the assumption

$$
0<\beta<\min \left\{\frac{1}{2}, \frac{1-\mu}{2-\mu}\right\}=\frac{1-\mu}{2-\mu},
$$

for any solution $(\varphi, \gamma)$ to the problem (3.13), it holds that for $x \in \mathbb{R}$,

$$
\begin{aligned}
|\varphi(x)| \leq & e^{-2 \mu \min _{1 \leq i \leq k}\left|x-\xi_{i}\right|}\left(K_{1}\|\varphi\|_{\mu}+C_{8}(\sigma)\|h\|_{\mu}\right) \\
& +e^{-\mu \min _{1 \leq i \leq k}\left|x-\xi_{i}\right|}\left(C_{11}(\sigma)\|\varphi\|_{\mu}+K_{2}\|h\|_{\mu}\right),
\end{aligned}
$$


where $K_{1} \geq 18, K_{2}>0$ are constants, and

$$
\begin{aligned}
C_{8}(\sigma) & =O\left(\sigma^{(1-\mu)(1-\beta)-\beta}|\log \sigma|\right), \\
C_{11}(\sigma) & =O\left(\sigma^{1-\frac{3 \beta}{2}+\frac{1}{4}(1-\mu)(1-\beta)}\right) .
\end{aligned}
$$

Proof. First of all, note that the assumption made in (3.63) is necessary due to the assumption (3.36) required in Lemma 3.3 and earlier proofs, as well as the assumption (3.58) required in (3.56) and (3.57).

We assemble (3.43), (3.44), (3.46), (3.52), (3.56) and (3.61) altogether to reach the following inequality,

$$
\begin{gathered}
|\varphi(x)| \leq\left|J^{1}\right|+\left|J^{2}\right|+\left|J^{3}\right|+\left|\frac{1}{2} \int_{\mathbb{R}} e^{-|x-y|} h(y) d y\right| \\
\leq e^{-2 \mu \rho(x)}\left[C_{12}\|\varphi\|_{\mu}+C_{8}(\sigma)\|h\|_{\mu}+C_{9}(\sigma)\|\varphi\|_{\mu}\right] \\
+e^{-\mu \rho(x)}\left[C_{10}(\sigma)\|h\|_{\mu}+C_{11}(\sigma)\|\varphi\|_{\mu}+\frac{1}{1-\mu}\|h\|_{\mu}\right] \\
=e^{-2 \mu \min _{1 \leq i \leq k}\left|x-\xi_{i}\right|}\left[C_{8}(\sigma)\|h\|_{\mu}+\left(C_{12}+C_{9}(\sigma)\right)\|\varphi\|_{\mu}\right] \\
+e^{-\mu \min _{1 \leq i \leq k}\left|x-\xi_{i}\right|}\left[\left(\frac{1}{1-\mu}+C_{10}(\sigma)\right)\|h\|_{\mu}+C_{11}(\sigma)\|\varphi\|_{\mu}\right], \forall x \in \mathbb{R},
\end{gathered}
$$

where the constant $C_{12}$ comes from (3.52) for $\left|J^{1}\right|$, with

$$
C_{12} \geq 18 C_{5}+144 \sigma^{1 / 12}+15 \sqrt{2} C_{5} \sigma^{\frac{3}{4}(1-\mu)(1-\beta)}\left(C_{0}+|\log \sigma|\right)
$$

and the other relevant constants are introduced in the estimates of $\left|J^{2}\right|$ and $\left|J^{3}\right|$. Finally, let

$$
\begin{aligned}
& K_{1}\left(\geq C_{12}+C_{9}(\sigma)\right) \text { be a constant, } K_{1} \geq 18 C_{5} \geq 18, \text { and } \\
& K_{2}\left(\geq \frac{1}{1-\mu}+C_{10}(\sigma)\right) \text { be a constant, }
\end{aligned}
$$

then we reach the conclusion of this lemma.

REMARK 2 (The order of $k=O\left(\sigma^{-\beta}\right)$ and the effect of $\mu$ ). According to the proof procedure we have accomplished so far, the assumption (3.63) on the order of $k=O\left(\sigma^{-\beta}\right)$,

$$
0<\beta<\frac{1-\mu}{2-\mu}\left(<\frac{1}{2}\right)
$$

is sharp. As we choose $\mu=\frac{1}{3}$, then accordingly $0<\beta<\frac{2}{5} \in\left(\frac{1}{3}, \frac{1}{2}\right)$. But we can reduce the parameter $\mu>0$ of the weighted norm to increase the number $\frac{1-\mu}{2-\mu}$ and to make it very close to $1 / 2$. We shall make another remark on the order $\beta$ after we finish the estimates in the nonlinear part later in Section 6.

Lemma 3.5. Under the assumption (3.63), if sequences $\left\{\sigma_{n}\right\} \subset \mathbb{R}^{+}$and $\left\{h_{n}\right\} \subset$ $L_{\mu}^{\infty}$ satisfy $0<\sigma_{n} \rightarrow 0$ and $\left\|h_{n}\right\|_{\mu} \rightarrow 0$, as $n \rightarrow \infty$, and if $\left(\varphi_{n}, \gamma^{n}\right) \in L_{\mu}^{\infty} \times \mathbb{R}^{k}$ is a solution to the problem (3.13), then it holds that

$$
\left\|\varphi_{n}\right\| \rightarrow 0, \text { as } n \rightarrow \infty \text {. }
$$


Proof. Suppose (3.69) does not hold. Without loss of generality, we can assume that

$$
\left\|\varphi_{n}\right\|_{\mu}=1, \text { for all } n \text {. }
$$

Substituting $\varphi=\varphi_{n}, h=h_{n}$, and $\sigma=\sigma_{n}$ into (3.64), in Lemma 3.4, and then taking the limit as $n \rightarrow \infty$, we get

$$
\left|\varphi_{n}(x)\right| \leq K_{1} e^{-2 \mu \min _{1 \leq i \leq k}\left|x-\xi_{i}\right|}, \quad x \in \mathbb{R}
$$

or equivalently,

$$
e^{\mu \min _{1 \leq i \leq k}\left|x-\xi_{i}\right|}\left|\varphi_{n}(x)\right| \leq K_{1} e^{-\mu \min _{1 \leq i \leq k}\left|x-\xi_{i}\right|}, \quad x \in \mathbb{R} .
$$

We claim that there exists at least one index $m \in\{1, \ldots, k\}$ such that

$$
\sup \left\{\left|\varphi_{n}(x)\right|: x \in I_{m}=\left[\xi_{m}-\frac{\log K_{1}}{\mu}, \xi_{m}+\frac{\log K_{1}}{\mu}\right]\right\} \geq \frac{1}{K_{1}} .
$$

Otherwise, for any

$$
x \in \bigcup_{j=1}^{k}\left[\xi_{j}-\frac{\log K_{1}}{\mu}, \xi_{j}+\frac{\log K_{1}}{\mu}\right]
$$

we would have

$$
e^{\mu \min _{1 \leq i \leq k}\left|x-\xi_{i}\right|}\left|\varphi_{n}(x)\right|<\frac{K_{1}}{K_{1}}=1 .
$$

Moreover, for any

$$
x \in \mathbb{R} \backslash \bigcup_{j=1}^{k}\left[\xi_{j}-\frac{\log K_{1}}{\mu}, \xi_{j}+\frac{\log K_{1}}{\mu}\right],
$$

by (3.71) we have

$$
e^{\mu \min _{1 \leq i \leq k}\left|x-\xi_{i}\right|}\left|\varphi_{n}(x)\right| \leq K_{1} e^{-\mu \min _{1 \leq i \leq k}\left|x-\xi_{i}\right|}<\frac{K_{1}}{K_{1}}=1 .
$$

Then it follows that

$$
e^{\mu \min _{1 \leq i \leq k}\left|x-\xi_{i}\right|}\left|\varphi_{n}(x)\right|<1, \quad \text { for every } x \in \mathbb{R},
$$

which contradicts the assumption $\left\|\varphi_{n}\right\|_{\mu}=1$. (Note that the norm $\left\|\varphi_{n}\right\|_{\mu}$ must be attained on a subset of $\mathbb{R}$ with a positive Lebesgue measure.) Therefore, the claim (3.72) holds. However, the index $m$ may depend on $n$.

Define the translated function $\tilde{\varphi}_{n}(x)=\varphi_{n}\left(x+\xi_{m}\right), x \in \mathbb{R}$, where $\xi_{m}$ is specified in (3.72). Then for all $n \geq 1$, one has

$$
\sup _{|x| \leq \frac{1}{\mu} \log K_{1}}\left|\tilde{\varphi}_{n}(x)\right| \geq \frac{1}{K_{1}} .
$$

Note that

$$
\frac{1}{\mu} \log K_{1}>0 \text { due to } K_{1} \geq 18 .
$$

On the other hand, (3.70) implies that

$$
0 \leq\left|\tilde{\varphi}_{n}(x)\right| \leq K_{1}, \quad x \in \mathbb{R} .
$$

By the Heine-Borel Theorem on compactness and by a diagonal sequence selection, we can confirm that there exists a subsequence of $\left\{\tilde{\varphi}_{n}\right\}$ which converges uniformly over compact intervals to a limit function denoted by $\tilde{\varphi}(x), x \in \mathbb{R}$. We relabel the 
convergent subsequence as the same as $\left\{\tilde{\varphi}_{n}\right\}$. Since $\tilde{\varphi}(x)$ satisfies Eq. (3.13), we have

$$
L\left(\tilde{\varphi}_{n}\right)=\widetilde{h_{n}}+\sum_{j=1}^{h} \gamma_{j}^{n} \tilde{W}_{j}, \quad x \in \mathbb{R}
$$

here $\widetilde{h_{n}}(x)=h_{n}\left(x+\xi_{m}\right)$ and $\tilde{W}_{j}=W_{j}\left(x+\xi_{m}\right)$. Thus $\tilde{\varphi}_{n}$ satisfies the integral equation

$$
\tilde{\varphi}_{n}(x)=\frac{1}{2} \int_{\mathbb{R}} e^{-|x-y|}\left(2 \tilde{W} \tilde{\varphi}_{n}-\frac{2 \tilde{W}^{2}}{\omega}\left\langle\tilde{W}, \tilde{\varphi}_{n}\right\rangle+\widetilde{h_{n}}+\gamma^{n} \cdot \nabla_{\xi} \tilde{W}\right) d y,
$$

where $\tilde{W}(x)=W\left(x+\xi_{m}\right)$. Now take limit as $n \rightarrow \infty$ and use the Dominated Convergence Theorem to see that the limit function $\tilde{\varphi}$ satisfies the equation

$$
\tilde{\varphi}(x)=\frac{1}{2} \int_{\mathbb{R}} e^{-|x-y|}(2 \tilde{W} \tilde{\varphi})(y) d y=\int_{\mathbb{R}} e^{-|x-y|} \tilde{W}(y) \tilde{\varphi}(y) d y, \quad x \in \mathbb{R},
$$

because the remaining terms in (3.74) converge to zero, namely,

$$
\begin{aligned}
& \int_{\mathbb{R}} e^{-|x-y|}\left(-\frac{2 \tilde{W}^{2}}{\omega}\left\langle\tilde{W}, \tilde{\varphi}_{n}\right\rangle\right) d y \rightarrow 0 \text { because of Lemma } 3.3, \\
& \int_{\mathbb{R}} e^{-|x-y|} \widetilde{h_{n}}(y) d y \rightarrow 0 \text { by the assumption }\left\|h_{n}\right\|_{\mu} \rightarrow 0, \text { and } \\
& \int_{\mathbb{R}} e^{-|x-y|} \gamma^{n} \cdot \nabla_{\xi} \tilde{W} d y \rightarrow 0 \text { because of Lemma } 3.2 .
\end{aligned}
$$

It follows that $\tilde{\varphi}$ is a solution to the problem:

$$
\begin{gathered}
L_{1}(\tilde{\varphi})=-\tilde{\varphi}^{\prime \prime}+(1-2 W) \tilde{\varphi}=0, \quad x \in \mathbb{R}, \\
\left\langle\tilde{\varphi}, \tilde{W}_{j}\right\rangle=0, \quad j=1, \ldots, k, \text { and } \\
\tilde{\varphi}(x) \rightarrow 0 \text { exponentially, as }|x| \rightarrow \infty .
\end{gathered}
$$

By Lemma 1.4, we know that

$$
\operatorname{Ker} L_{1}=\operatorname{Span}\left\{W_{1}, \ldots, W_{k}\right\}=\operatorname{Span}\left\{\tilde{W}_{1}, \ldots, \tilde{W}_{k}\right\} .
$$

Hence, (3.76) implies that

$$
\tilde{\varphi} \in \operatorname{Ker} L_{1} \cap\left(\operatorname{Ker} L_{1}\right)^{\perp}=\{0\}, \quad \text { i.e., } \tilde{\varphi}(x) \equiv 0 .
$$

However, $\tilde{\varphi}_{n}$ also satisfies the equation

$$
\tilde{\varphi}_{n}=(-\Delta+I)^{-1}\left[2 \tilde{W} \tilde{\varphi}_{n}-\frac{2 \tilde{W}^{2}}{\omega}\left\langle\tilde{W}, \tilde{\varphi}_{n}\right\rangle+\widetilde{h_{n}}+\gamma^{n} \cdot \nabla_{\xi} \tilde{W}\right]
$$

by the regularity of the solutions to harmonic equations, this implies $\tilde{\varphi}_{n} \in H^{2}(\mathbb{R}) \cap$ $H_{0}^{1}(\mathbb{R})$ and

$$
\left\|\tilde{\varphi}_{n}\right\|_{H^{2}(\mathbb{R})} \leq \text { const, for } n \geq 1 \text {. }
$$

The Sobolev imbedding property $H^{2}(\mathbb{R}) \rightarrow C_{\text {loc }}^{0,1}(\mathbb{R})$ infers that $\left\{\tilde{\varphi}_{n}\right\}$ is uniformly Lipschitz continuous on the compact interval $I_{0}=\left[-\mu^{-1} \log K_{1}\right.$, $\left.\mu^{-1} \log K_{1}\right]$. This together with (3.73) implies that there is a positive number $\nu_{0}>0$ such that

$$
\operatorname{meas}\left\{x \in I_{0}:\left|\tilde{\varphi}_{n}(x)\right| \geq \frac{1}{2 K_{1}}\right\} \geq \nu_{0}
$$


Therefore, there exists a measurable subset $\tilde{I} \subset I_{0} \subset \mathbb{R}$, such that meas $(\tilde{I})>0$ and

$$
\tilde{\varphi}(x)>0 \text { for } x \in \tilde{I} \text {. }
$$

Finally, (3.77) and (3.78) constitute a contradiction. This contradiction shows that (3.69) holds. The lemma is proved.

Now we can prove the key result concerning the solution of the augmented linear problem (3.13), which is the principal approximation of the linear part of the original equation (2.14).

THEOREM 3.6. Under the assumption

$$
k=\operatorname{const} \sigma^{-\beta} \text {, with } 0<\beta<\frac{1-\mu}{2-\mu},
$$

there exists a positive number $\hat{\sigma} \leq \frac{1}{2}$ such that for any given $0<\sigma \leq \hat{\sigma}$ and for any $\xi=\left(\xi_{1}, \ldots, \xi_{k}\right) \in \Lambda$ in (1.16) and a given $h \in L_{\mu}^{\infty}$, the problem (3.13) has a unique solution $(\varphi, \gamma)$, which is denoted by $\varphi=\mathcal{J}(h)$ and $\gamma=\Pi(h)$. Moreover, there exist two positive constants $\Gamma_{0}$ and $\Gamma_{1}$ such that

$$
\begin{aligned}
\|\varphi\|_{\mu} & =\|\mathcal{J}(h)\|_{\mu} \leq \Gamma_{0}\|h\|_{\mu}, \\
\|\gamma\|_{\mathbb{R}^{k}} & =\|\Pi(h)\|_{\mathbb{R}^{k}} \leq \Gamma_{1}\|h\|_{\mu} .
\end{aligned}
$$

Proof. Define a Hilbert space $H$ by

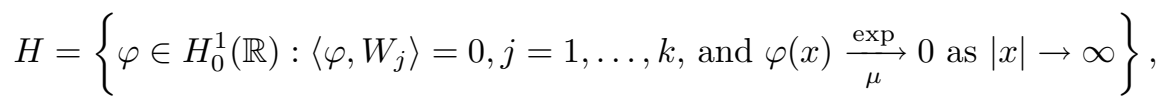

where $\varphi(x) \underset{\mu}{\stackrel{\exp }{\longrightarrow}} 0$, as $|x| \rightarrow \infty$, means that

$$
\limsup _{|x| \rightarrow \infty} e^{\mu|x|}|\varphi(x)|<\infty
$$

and $H$ is endowed with the norm of Sobolev space $H_{0}^{1}(\mathbb{R})$. A solution to the problem (3.13) ought to be such a pair $(\varphi, \gamma)$ that $\varphi \in H$ satisfying the equation

$$
\int_{\mathbb{R}}\left(\varphi^{\prime} \psi^{\prime}+\varphi \psi\right) d y-\langle 2 W \varphi, \psi\rangle+\frac{2}{\omega}\langle W, \varphi\rangle\left\langle W^{2}, \psi\right\rangle=\langle h, \psi\rangle, \forall \psi \in H,
$$

and vice-versa, and $\gamma \in \mathbb{R}^{k}$ satisfying the equation (3.16). Note that this variational equation (3.81) is equivalent to the following version,

$$
\varphi-(1-\Delta)^{-1} R(\varphi)=(1-\Delta)^{-1} h,
$$

where $R(\varphi)=2 W \varphi-\frac{2 W^{2}}{\omega}\langle W, \varphi\rangle$. Since $(I-\Delta)^{-1}$ is a compact linear operator and $R$ is a bounded linear operator, so that $(I-\Delta)^{-1} R$ is a compact operator on $H$. And $(I-\Delta)^{-1} h \in H$. Thus by the Fredholm alternative principle, Eq. (3.81) is uniquely solvable if and only if it admits only the trivial zero solution for $h=0$.

Suppose the existence and uniqueness part of this theorem does not hold, then there exists a positive sequence $\left\{\sigma_{n}\right\}, \sigma_{n} \rightarrow 0$, as $n \rightarrow \infty$, and a corresponding solution sequence $\left\{\varphi_{n}\right\} \subset H$ of the problem (3.13) with $h_{n} \equiv 0$, such that $\left\|\varphi_{n}\right\|_{\mu}=1$ for $n \geq 1$, since we can always normalize a nontrivial solution $\varphi_{n}$ with $\left\|\varphi_{n}\right\|_{\mu} \neq$ 0. However, this directly contradicts Lemma 3.5 as we have shown. Therefore, applying the Fredholm alternative principle and Lemma 3.2, we can assert that 
problem (3.13) has a unique solution for any $0<\sigma \leq \hat{\sigma}$, where $\hat{\sigma}$ is some constant sufficiently small.

To show the boundedness of the solution mapping $\varphi=\mathcal{J}(h)$ and $\gamma \in \Pi(h)$ in (3.80), we can use (3.82). By the inverse operator theorem in Banach spaces, the unique solvability we proved in the first part of this theorem implies that

$$
\left[I-(I-\Delta)^{-1} R\right]^{-1} \text { is a bounded linear operator, }
$$

so that the linear operator $\mathcal{J}$ for the $\varphi$-component,

$$
\mathcal{J}=\left[I-(I-\Delta)^{-1} R\right]^{-1}(I-\Delta)^{-1} \in \mathcal{L}\left(L_{\mu}^{\infty}, L_{\mu}^{\infty}\right)
$$

is bounded. Then by Lemma 3.2, the linear operator $\Pi$ for the $\gamma$-component is also bounded. The proof is completed.

\section{Nonlinear Estimates}

In this section, we have two objectives. First we shall take the full consideration of the $\sigma$ effect in $T[W \varphi]$, which is replaced by $\frac{1}{\omega}\langle W, \varphi\rangle$ in Section 3 , and estimate the difference between $V(x)$ and constant 1, while we set $V(x)=1$ in Section 3 . Second is a key result on the estimate of the integrals

$$
\int_{\mathbb{R}} S[W](x) U^{\prime}\left(x-\xi_{j}\right) d x, \quad j=1, \ldots, k,
$$

which constitutes the main part of the nonlinear side in (2.14). Here, $W(x)$ defined by (1.17), $\Lambda$ defined by (1.16) and $L_{\mu}^{\infty}$ defined by (3.12) remain the same.

I) For each given $\xi=\left(\xi_{1}, \ldots, \xi_{k}\right) \in \Lambda$ and $W(x)$ given in (1.17) accordingly, the function $V=T\left[W^{2}\right]$ is the unique solution of the ODE problem (2.11), which has the explicit expression (2.12). We first want to know the behavior of this function $V$ around the points $\xi_{1}, \ldots, \xi_{k}$. For each $\ell=1, \ldots, k$, we have

$$
\begin{gathered}
V\left(\xi_{\ell}\right)=\frac{1}{\omega} \int_{\mathbb{R}} e^{-\sigma\left|\xi_{\ell}-x\right|}\left[\sum_{j=1}^{k} U^{2}\left(x-\xi_{j}\right)+\sum_{\substack{i, j \\
i \neq j}} U\left(x-\xi_{i}\right) U\left(x-\xi_{j}\right)\right] d x \\
=1+\frac{1}{\omega} \int_{\mathbb{R}}\left(e^{-\sigma\left|\xi_{\ell}-x\right|}-1\right)\left(\sum_{j=1}^{k} U^{2}\left(x-\xi_{j}\right)+\sum_{\substack{i, j \\
i \neq j}} U\left(x-\xi_{i}\right) U\left(x-\xi_{j}\right)\right) d x
\end{gathered}
$$

where

$$
\omega=\int_{\mathbb{R}} W^{2}(x) d x, \text { as we introduced in }(2.16) .
$$

By the instrumental inequality

$$
0 \leq 1-e^{-\sigma y} \leq \sigma y, \quad \text { which is valid for all } y \geq 0,
$$

we can make the following estimates, in which $C$ may represent different positive constants which need not be further specified. 
A) For $j \neq \ell$ in the first sum in (4.1), we have

$$
\begin{gathered}
\left|\int_{\mathbb{R}}\left(e^{-\sigma\left|\xi_{\ell}-x\right|}-1\right) U^{2}\left(x-\xi_{j}\right) d x\right| \leq \int_{\mathbb{R}}\left|1-e^{-\sigma\left|\xi_{\ell}-\xi_{j}-x\right|}\right| U^{2}(x) d x \\
\leq \int_{\mathbb{R}} \sigma\left|\xi_{\ell}-\xi_{j}-x\right| U^{2}(x) d x \leq \sigma\left(\int_{\mathbb{R}}|x| U^{2}(x) d x+\int_{\mathbb{R}}\left|\xi_{\ell}-\xi_{j}\right| U^{2}(x) d x\right) \\
=\sigma\left(12 \log 2-3+6\left|\xi_{\ell}-\xi_{j}\right|\right) \quad(\text { by Lemma } 1.1) \\
\leq \sigma(12 \log 2-3+6|\ell-j||\log \sigma|) \leq \sigma(12 \log 2-3+6(k-1)|\log \sigma|) \\
\leq C \sigma^{1-\beta}|\log \sigma|, \quad \text { for some constant } C>0 .
\end{gathered}
$$

Hence,

(4.4)

$$
\begin{gathered}
\left|\int_{\mathbb{R}}\left(e^{-\sigma\left|\xi_{\ell}-x\right|}-1\right) \sum_{j=1}^{k} U^{2}\left(x-\xi_{j}\right) d x\right| \leq\left|\int_{\mathbb{R}}\left(e^{-\sigma\left|\xi_{\ell}-x\right|}-1\right) U^{2}\left(x-\xi_{\ell}\right) d x\right| \\
+\sum_{j(\neq \ell)=1}^{k}\left|\int_{\mathbb{R}}\left(e^{-\sigma\left|\xi_{\ell}-x\right|}-1\right) U^{2}\left(x-\xi_{j}\right) d x\right| \\
\leq \sigma \int_{\mathbb{R}}|x| U^{2}(x) d x+(k-1) C \sigma^{1-\beta}|\log \sigma| \\
\leq(12 \log 2-3) \sigma+(k-1) C \sigma^{1-\beta}|\log \sigma|
\end{gathered}
$$

B) For those cross-product integral terms with either $i=\ell \neq j$ or $j=\ell \neq i$, we have

$(4.5)$

$$
\begin{aligned}
& \sum_{i=\ell \neq j \text { or } j=\ell \neq i}\left|\int_{\mathbb{R}}\left(e^{-\sigma\left|\xi_{\ell}-x\right|}-1\right) U\left(x-\xi_{i}\right) U\left(x-\xi_{j}\right) d x\right| \\
& \leq 2 \sum_{j(\neq \ell)=1}^{k} \int_{\mathbb{R}}\left|e^{-\sigma|y|}-1\right| U(y) U\left(y+\xi_{\ell}-\xi_{j}\right) d x \\
& \leq 72 \sigma \sum_{j(\neq \ell)=1}^{k} \int_{\mathbb{R}}|y| e^{-|y|} e^{-\left|y+\xi_{\ell}-\xi_{j}\right|} d y \quad \text { (by using (1.8)) } \\
& =36 \sigma \sum_{j(\neq \ell)=1}^{k} e^{-\left|\xi_{\ell}-\xi_{j}\right|}\left(1+\left|\xi_{\ell}-\xi_{j}\right|+\left|\xi_{\ell}-\xi_{j}\right|^{2}\right)
\end{aligned}
$$

$\leq 36 \sigma \sum_{j(\neq \ell)=1}^{k} \sigma^{|\ell-j|(1-\beta)}\left(1+|\ell-j||\log \sigma|+|\ell-j|^{2}|\log \sigma|^{2}\right) \quad$ (by Lemma 1.3)

$$
\begin{gathered}
\leq 36 \sigma\left[C \sigma^{1-\beta}+C \sigma^{1-\beta}|\log \sigma|+k|\log \sigma| C \sigma^{1-\beta}|\log \sigma|\right] \\
\leq C \sigma^{2-\beta}(1+|\log \sigma|)+C \sigma^{2(1-\beta)}|\log \sigma|^{2} \\
=O\left(\sigma^{2(1-\beta)}|\log \sigma|^{2}\right) .
\end{gathered}
$$


C) For those cross-product integral terms with $(i, j, \ell)$ mutually unequal, we have

$$
\begin{gathered}
\sum_{\substack{i, j(\neq \ell) \\
i \neq j}}\left|\int_{\mathbb{R}}\left(e^{\left|\xi_{\ell}-x\right|}-1\right) U\left(x-\xi_{i}\right) U\left(x-\xi_{j}\right) d x\right| \\
\leq 36 \sigma \sum_{\substack{i, j(\neq \ell) \\
i \neq j}} \int_{\mathbb{R}}\left|\xi_{\ell}-\xi_{i}-x\right| e^{-|x|} e^{-\left|x+\xi_{i}-\xi_{j}\right|} d x \\
\leq 36 \sigma \sum_{\substack{i, j(\neq \ell) \\
i \neq j}}\left\{\left|\xi_{\ell}-\xi_{i}\right| \int_{\mathbb{R}} e^{-|x|} e^{-\left|x+\xi_{i}-\xi_{j}\right|} d x+\int_{\mathbb{R}}|x| e^{-|x|} e^{-\left|x+\xi_{i}-\xi_{j}\right|} d x\right\}
\end{gathered}
$$

(by (1.7) and (1.8))

$$
\begin{gathered}
\leq 36 \sigma \sum_{i, j(\neq \ell)}\left\{|\ell-i||\log \sigma|\left(1+\left|\xi_{i}-\xi_{j}\right|\right) e^{-\left|\xi_{i}-\xi_{j}\right|}\right. \\
\left.+\frac{1}{2} e^{-\left|\xi_{i}-\xi_{j}\right|}\left(1+\left|\xi_{i}-\xi_{j}\right|+\left|\xi_{i}-\xi_{j}\right|^{2}\right)\right\} \\
\leq 36 \sigma \sum_{\substack{i, j \\
i \neq j}} e^{-\left|\xi_{i}-\xi_{j}\right|}\left[\frac{1}{2}\left(1+\left|\xi_{i}-\xi_{j}\right|\right)+k|\log \sigma|\left(1+2\left|\xi_{i}-\xi_{j}\right|\right)\right] \\
\leq 36 \sigma \sum_{\substack{i, j \\
i \neq j}} \sigma^{|i-j|(1-\beta)}\left[\frac{1}{2}(1+|i-j||\log \sigma|)+k|\log \sigma|(1+2|i-j||\log \sigma|)\right]
\end{gathered}
$$

(by Lemma 1.3)

$$
\begin{gathered}
\leq 36 \sigma k\left[C \sigma^{1-\beta}(1+|\log \sigma|)+k|\log \sigma| C \sigma^{1-\beta}|\log \sigma|\right] \\
\leq C \sigma^{2(1-\beta)}(1+|\log \sigma|)+C \sigma^{2-3 \beta}|\log \sigma|^{2} \\
=O\left(\sigma^{2-3 \beta}|\log \sigma|^{2}\right) .
\end{gathered}
$$

Again, note that

$$
\omega=\int_{\mathbb{R}} W^{2}(x) d x \geq 6 k, \text { and } k=O\left(\sigma^{-\beta}\right) .
$$

From (4.1), (4.4), (4.5), (4.6) and (4.7) we obtain

$$
\begin{gathered}
V\left(\xi_{\ell}\right)-1 \leq \frac{1}{6 k}\left\{(12 \log 2-3) \sigma+(k-1) C \sigma^{1-\beta}|\log \sigma|\right. \\
\left.+O\left(\sigma^{2(1-\beta)}|\log \sigma|^{2}\right)+O\left(\sigma^{2-3 \beta}|\log \sigma|^{2}\right)\right\} \\
=O\left(\sigma^{1+\beta}\right)+O\left(\sigma^{1-\beta}|\log \sigma|\right)+O\left(\sigma^{2-\beta}|\log \sigma|^{2}\right)+O\left(\sigma^{2(1-\beta)}|\log \sigma|^{2}\right) \\
=O\left(\sigma^{1-\beta}|\log \sigma|\right) .
\end{gathered}
$$

Therefore, we have the following lemma.

LEMMA 4.1. It holds that

$$
V\left(\xi_{\ell}\right)=1+O\left(\sigma^{1-\beta}|\log \sigma|\right), \quad \ell=1, \ldots, k .
$$


II) In order to know the information about $V\left(\xi_{\ell}+y\right)$, for $|y| \leq \frac{1}{2}|\log \sigma|, \ell=$ $1, \ldots, k$, which covers the interval $\left[\xi_{1}-\frac{1}{2}|\log \sigma|, \xi_{k}+\frac{1}{2}|\log \sigma|\right]$ of the real line, we now estimate the increment $V\left(\xi_{\ell}+y\right)-V\left(\xi_{\ell}\right)$. By (4.1), we have

$$
\begin{gathered}
V\left(\xi_{\ell}+y\right)-V\left(\xi_{\ell}\right)=\frac{1}{\omega} \int_{\mathbb{R}}\left[e^{-\sigma\left|\xi_{\ell}+y-x\right|}-e^{-\sigma\left|\xi_{\ell}-x\right|}\right] \\
\times\left[\sum_{j=1}^{k} U^{2}\left(x-\xi_{j}\right)+\sum_{\substack{i, j \\
i \neq j}} U\left(x-\xi_{i}\right) U\left(x-\xi_{j}\right)\right] d x .
\end{gathered}
$$

The estimates will be made part by part, as follows.

a) For $j<\ell$ in the first sum, by using the Taylor expansion

$$
e^{-x}=1-x+O\left(x^{2}\right), \quad \text { for } x \text { (small) }>0,
$$

we have

$$
\begin{gathered}
\int_{\mathbb{R}}\left[e^{-\sigma\left|\xi_{\ell}+y-x\right|}-e^{-\sigma\left|\xi_{\ell}-x\right|}\right] U^{2}\left(x-\xi_{j}\right) d x \\
=\int_{\mathbb{R}}\left[e^{-\sigma\left|\xi_{\ell}-\xi_{j}+y-x\right|}-e^{-\sigma\left|\xi_{\ell}-\xi_{j}-x\right|}\right] U^{2}(x) d x \\
=\int_{\mathbb{R}}\left[e^{-\sigma\left(\xi_{\ell}-\xi_{j}+y-x\right)}-e^{-\sigma\left(\xi_{\ell}-\xi_{j}-x\right)}\right] U^{2}(x) d x+R(y) \\
=e^{-\sigma\left(\xi_{\ell}-\xi_{j}\right)} \int_{\mathbb{R}}\left[e^{-\sigma(y-x)}-e^{\sigma x}\right] U^{2}(x) d x+R(y) \\
=e^{-\sigma\left|\xi_{\ell}-\xi_{j}\right|}\left(e^{-\sigma y}-1\right) \int_{\mathbb{R}} e^{\sigma x} U^{2}(x) d x+R(y) \\
=e^{-\sigma\left|\xi_{\ell}-\xi_{j}\right|}\left[-\sigma y+O\left(\sigma^{2} y^{2}\right)\right] \hat{C}(\sigma)+R(y),
\end{gathered}
$$

where the constant $\hat{C}(\sigma)$ is given by

$$
\hat{C}(\sigma) \triangleq \int_{\mathbb{R}} e^{\sigma x} U^{2}(x) d x \leq 36\left(\frac{1}{2-\sigma}+\frac{1}{2+\sigma}\right)=\frac{144}{4-\sigma^{2}}<40,
$$

and the remainder $R(y)$ can be expressed as

$$
\begin{aligned}
R(y) & =\int_{y+\xi_{\ell}-\xi_{j}}^{\infty}\left[e^{-\sigma\left(x-y+\xi_{j}-\xi_{\ell}\right)}-e^{-\sigma\left(\xi_{\ell}-\xi_{j}+y-x\right)}\right] U^{2}(x) d x \\
& -\int_{\xi_{\ell}-\xi_{j}}^{\infty}\left[e^{-\sigma\left(x+\xi_{j}-\xi_{\ell}\right)}-e^{-\sigma\left(\xi_{\ell}-\xi_{j}-x\right)}\right] U^{2}(x) d x,
\end{aligned}
$$

where the two integrals in (4.13) will be denoted by $R_{1}(y)$ and $R_{2}(y)$, respectively. Then,

$$
\begin{gathered}
\left|R_{1}(y)\right|=\left|\int_{y+\xi_{\ell}-\xi_{j}}^{\infty}\left[e^{-\sigma\left(x-y+\xi_{j}-\xi_{\ell}\right)}-e^{\sigma\left(x-y+\xi_{j}-\xi_{\ell}\right)}\right] U^{2}(x) d x\right| \\
\leq \int_{y+\xi_{\ell}-\xi_{j}}^{\infty}\left|-2 \sigma\left(x-y+\xi_{j}-\xi_{\ell}\right)\right| U^{2}(x) d x+\text { (h.o.t.) } \\
\leq 72 \sigma\left\{\int_{y+\xi_{\ell}-\xi_{j}}^{\infty}(|x|+|y|) e^{-2 x} d x+|j-\ell||\log \sigma| \int_{y+\xi_{\ell}-\xi_{j}}^{\infty} e^{-2 x} d x\right\}+\text { (h.o.t.) }
\end{gathered}
$$


in which (h.o.t.) stands for higher-order terms. Since we have, for $j<\ell$, that

$$
\begin{gathered}
\int_{y+\xi_{\ell}-\xi_{j}}^{\infty}(|x|+|y|) e^{-2 x} d x=\int_{y+\xi_{\ell}-\xi_{j}}^{\infty} x e^{-2 x} d x+|y| \int_{y+\xi_{\ell}-\xi_{j}}^{\infty} e^{-2 x} d x \\
=\left.\left(-\frac{x}{2} e^{-2 x}-\frac{1}{4} e^{-2 x}\right)\right|_{y+\xi_{\ell}-\xi_{j}} ^{\infty}+\left.|y|\left(-\frac{1}{2} e^{-2 x}\right)\right|_{y+\xi_{\ell}-\xi_{j}} ^{\infty} \\
\leq \frac{1}{2}(2|y|+|\ell-j||\log \sigma|+1) e^{-2\left(y+\xi_{\ell}-\xi_{j}\right)} \\
\leq \frac{1}{2}(1+(\ell-j+1)|\log \sigma|) \sigma^{2(\ell-j)(1-\beta)-1},
\end{gathered}
$$

because $|y| \leq \frac{1}{2}|\log \sigma|$, and

$$
\int_{y+\xi_{\ell}-\xi_{j}}^{\infty} e^{-2 x} d x \leq \frac{1}{2} \sigma^{2(\ell-j)(1-\beta)-1} .
$$

Therefore,

$$
\begin{aligned}
\left|R_{1}(y)\right| & \leq 36 \sigma^{2(\ell-j)(1-\beta)}[1+(2 \ell-2 j+1)|\log \sigma|]+\text { (h.o.t.) } \\
& \leq C \sigma^{2(\ell-j)(1-\beta)}(\ell-j+1)|\log \sigma| .
\end{aligned}
$$

Similarly, we can get

$$
\begin{gathered}
\left|R_{2}(y)\right|=\left|\int_{\xi_{\ell}-\xi_{j}}^{\infty}\left[e^{-\sigma\left(x+\xi_{j}-\xi_{\ell}\right)}-e^{\sigma\left(x+\xi_{j}-\xi_{\ell}\right)}\right] U^{2}(x) d x\right| \\
\left.\leq 72 \sigma\left\{\int_{\xi_{\ell}-\xi_{j}}^{\infty} x e^{-2 x} d x+(\ell-j)|\log \sigma| \int_{\xi_{\ell}-\xi_{j}}^{\infty} e^{-2 x} d x\right\}+\text { (h.o.t. }\right) \\
\leq C \sigma^{2(\ell-j)(1-\beta)+1}[1+(\ell-j)|\log \sigma|] .
\end{gathered}
$$

Substituting (4.15) and (4.16) into (4.13), we obtain that, for $j<\ell,|y| \leq \frac{1}{2}|\log \sigma|$,

$$
\int_{\mathbb{R}}\left[e^{-\sigma\left|\xi_{\ell}+y-x\right|}-e^{-\sigma\left|\xi_{\ell}-x\right|}\right] U^{2}\left(x-\xi_{j}\right) d x
$$

$$
=\hat{C}(\sigma) e^{-\sigma\left|\xi_{\ell}-\xi_{j}\right|}\left[-\sigma y+O\left(\sigma^{2}|\log \sigma|^{2}\right)\right]+O\left(\sigma^{2(\ell-j)(1-\beta)}(\ell-j+1)|\log \sigma|\right) .
$$

$$
\begin{gathered}
\int_{\mathbb{R}}\left[e^{-\sigma\left|\xi_{\ell}+y-x\right|}-e^{-\sigma\left|\xi_{\ell}-x\right|}\right] U^{2}\left(x-\xi_{j}\right) d x \\
=\int_{\mathbb{R}}\left[e^{-\sigma\left|\xi_{j}-\xi_{\ell}+x-y\right|}-e^{-\sigma\left|\xi_{j}-\xi_{\ell}+x\right|}\right] U^{2}(x) d x \\
=\int_{\mathbb{R}}\left[e^{-\sigma\left(\xi_{j}-\xi_{\ell}+x-y\right)}-e^{-\sigma\left(\xi_{j}-\xi_{\ell}+x\right)}\right] U^{2}(x) d x+\tilde{R}(y) \\
=\hat{C}(\sigma) e^{-\sigma\left|\xi_{j}-\xi_{\ell}\right|}\left[\sigma y+O\left(\sigma^{2}|\log \sigma|^{2}\right)\right]+O\left(\sigma^{2(j-\ell)(1-\beta)}(j-\ell+1)|\log \sigma|\right),
\end{gathered}
$$

for $|y| \leq \frac{1}{2}|\log \sigma|$, where $\hat{C}(\sigma)$ is the same constant in (4.12), and $\tilde{R}(y)$ represents the corresponding remainder which is treated similar to (4.13) through (4.16). 
c) For $j=\ell$ in the first sum in (4.9), we have

$$
\begin{gathered}
\int_{\mathbb{R}}\left[e^{-\sigma\left|\xi_{\ell}+y-x\right|}-e^{-\sigma\left|\xi_{\ell}-x\right|}\right] U^{2}\left(x-\xi_{\ell}\right) d x \\
=\int_{\mathbb{R}}\left[e^{-\sigma|y-x|}-e^{-\sigma|x|}\right] U^{2}(x) d x \\
=-\sigma \int_{\mathbb{R}}[|y-x|-|x|] U^{2}(x) d x+O\left(\sigma^{2}|\log \sigma|^{2}\right) .
\end{gathered}
$$

Let

$$
\Psi(y)=\int_{\mathbb{R}}[|y-x|-|x|] U^{2}(x) d x, \quad y \in \mathbb{R} .
$$

Since $\Psi$ satisfies

$$
\begin{aligned}
& \Psi^{\prime \prime}(y)=2 U^{2}(y), \quad y \in \mathbb{R}, \\
& \Psi(0)=0, \quad \Psi^{\prime}(0)=0,
\end{aligned}
$$

and $U^{2}(y)$ is an even function, $\Psi$ is also an even function as the solution of (4.21). Therefore, $\Psi U^{2} U^{\prime}$ is an odd function, so that

$$
\int_{\mathbb{R}} \Psi(y) U^{2}(y) U^{\prime}(y) d y=0 .
$$

This property will be used later.

d) Next let us estimate the cross-product integral terms in (4.9),

$$
\begin{gathered}
\int_{\mathbb{R}}\left[e^{-\sigma\left|\xi_{\ell}+y-x\right|}-e^{-\sigma\left|\xi_{\ell}-x\right|}\right] \sum_{\substack{i, j \\
i \neq j}} U\left(x-\xi_{i}\right) U\left(x-\xi_{j}\right) d x \\
=\sum_{j=1}^{k} \int_{\mathbb{R}}\left[e^{-\sigma\left|\xi_{\ell}+y-x\right|}-e^{-\sigma\left|\xi_{\ell}-x\right|}\right] \sum_{i(\neq j)=1}^{k} U\left(x-\xi_{i}\right) U\left(x-\xi_{j}\right) d x .
\end{gathered}
$$

For $j<\ell$, similarly we can get

$$
\begin{gathered}
\int_{\mathbb{R}}\left[e^{-\sigma\left|\xi_{\ell}+y-x\right|}-e^{-\sigma\left|\xi_{\ell}-x\right|}\right] \sum_{i(\neq j)=1}^{k} U\left(x-\xi_{i}\right) U\left(x-\xi_{j}\right) d x \\
=\tilde{C}(\sigma) e^{-\sigma\left|\xi_{\ell}-\xi_{j}\right|}\left[-\sigma y+O\left(\sigma^{2} y^{2}\right)\right]+\Phi^{j}(y)
\end{gathered}
$$

where the coefficient $\tilde{C}(\sigma)$ and the remainder $\Phi^{j}(y)$ have the following properties 
i) $\tilde{C}(\sigma)=\sum_{i(\neq j)=1}^{k} \tilde{C}_{i}(\sigma)$, with

$$
\begin{aligned}
\tilde{C}_{i}(\sigma) & =\left|\tilde{C}_{i}(\sigma)\right|=\int_{\mathbb{R}} e^{\sigma x} U(x) U\left(x+\xi_{j}-\xi_{i}\right) d x \\
& \leq 36 \int_{\mathbb{R}} e^{\sigma x} e^{-|x|} e^{-\left|x+\xi_{j}-\xi_{i}\right|} d x \quad \text { (by direct integration) } \\
& \leq 36\left(\frac{1}{2-\sigma}+\frac{1}{2+\sigma}+\left|\xi_{j}-\xi_{i}\right|\right) e^{-\left|\xi_{j}-\xi_{i}\right|} \\
& \leq 36\left(\frac{4}{4-\sigma^{2}}+|j-i||\log \sigma|\right) \sigma^{|j-i|(1-\beta)} \\
& <(48+36|j-i||\log \sigma|) \sigma^{|j-i|(1-\beta)}
\end{aligned}
$$

where we used the explicit expression of $p_{r}(x)$ shown in the proof of Lemma 1.2, so that

$$
\begin{aligned}
\tilde{C}(\sigma)= & |\tilde{C}(\sigma)| \leq \sum_{i(\neq j)=1}^{k}(48+36|j-i||\log \sigma|) \sigma^{|j-i|(1-\beta)} \\
& \leq C(1+|\log \sigma|) \sigma^{1-\beta}, \quad \text { for a constant } C .
\end{aligned}
$$

ii) Similar to (4.13) and (4.14), the remainder $\Phi^{j}$ can be decomposed into

$$
\begin{aligned}
\Phi^{j}(y) & =\int_{y+\xi_{\ell}-\xi_{j}}^{\infty}\left[e^{-\sigma\left(x-y+\xi_{j}-\xi_{\ell}\right)}-e^{\sigma\left(x-y+\xi_{j}-\xi_{\ell}\right)}\right] \sum_{i(\neq j)=1}^{k} U(x) U\left(x+\xi_{j}-\xi_{i}\right) d x \\
& -\int_{\xi_{\ell}-\xi_{j}}^{\infty}\left[e^{-\sigma\left(x+\xi_{j}-\xi_{\ell}\right)}-e^{\sigma\left(x+\xi_{j}-\xi_{\ell}\right)}\right] \sum_{i(\neq j)=1}^{k} U(x) U\left(x+\xi_{j}-\xi_{i}\right) d x
\end{aligned}
$$

where the two integrals in (4.26) will be denoted by $\Phi_{1}^{j}(y)$ and $\Phi_{2}^{j}(y)$, respectively. Then we have

$$
\begin{aligned}
& \left|\Phi_{1}^{j}(y)\right|=\left|\int_{y+\xi_{\ell}-\xi_{j}}^{\infty}\left[e^{-\sigma\left(x-y+\xi_{j}-\xi_{\ell}\right)}-e^{\sigma\left(x-y+\xi_{j}-\xi_{\ell}\right)}\right] \sum_{i(\neq j)=1}^{k} U(x) U\left(x+\xi_{j}-\xi_{i}\right) d x\right| \\
& \left.\leq 72 \sigma \int_{y+\xi_{\ell}-\xi_{j}}^{\infty}\left(|x|+|y|+\left|\xi_{j}-\xi_{\ell}\right|\right) \sum_{i(\neq j)=1}^{k} e^{-|x|} e^{-\left|x+\xi_{j}-\xi_{i}\right|} d x+\text { (h.o.t. }\right),
\end{aligned}
$$


where (h.o.t.) represents the higher-order terms, and by using Lemmas 1.2 and 1.3 we find that

$$
\begin{gathered}
\sum_{i(\neq j)=1}^{k} \int_{y+\xi_{\ell}-\xi_{j}}^{\infty}|x| e^{-|x|} e^{-\left|x+\xi_{j}-\xi_{i}\right|} d x \quad(\text { by }(1.8)) \\
\leq \sum_{i(\neq j)=1}^{k} \frac{1}{2} e^{-\left|\xi_{j}-\xi_{i}\right|}\left(1+\left|\xi_{j}-\xi_{i}\right|\right)^{2} \\
\leq \sum_{i(\neq j)=1}^{k} \frac{1}{2} \sigma^{|i-j|(1-\beta)}(1+|i-j||\log \sigma|)^{2} \\
\leq C \sigma^{1-\beta}|\log \sigma|^{2},
\end{gathered}
$$

and

$$
\begin{gathered}
\sum_{i(\neq j)=1}^{k} \int_{y+\xi_{\ell}-\xi_{j}}^{\infty}\left(|y|+\left|\xi_{\ell}-\xi_{j}\right|\right) e^{-|x|} e^{-\left|x+\xi_{j}-\xi_{i}\right|} d x \quad \text { by (1.7)) } \\
\leq \sum_{i(\neq j)=1}^{k}\left(\frac{1}{2}|\log \sigma|+|j-\ell||\log \sigma|\right) \int_{y+\xi_{\ell}-\xi_{j}}^{\infty} e^{-|x|} e^{-\left|x+\xi_{j}-\xi_{i}\right|} d x \\
\leq \sum_{i(\neq j)=1}^{k}\left(\frac{1}{2}|\log \sigma|+|j-\ell||\log \sigma|\right)(1+|i-j||\log \sigma|) \sigma^{|i-j|(1-\beta)} \\
\leq C(1+|j-\ell|)|\log \sigma|^{2} \sigma^{1-\beta} .
\end{gathered}
$$

Therefore, we obtain

$$
\left|\Phi_{1}^{j}(y)\right| \leq C \sigma^{2-\beta}(1+|j-\ell|)|\log \sigma|^{2}, \quad \text { for } j<\ell \text { and } j>\ell .
$$

Moreover, we have

$$
\begin{aligned}
\left|\Phi_{2}^{j}(y)\right|=\left|\int_{\xi_{\ell}-\xi_{i}}^{\infty}\left[e^{-\sigma\left(x+\xi_{j}-\xi_{\ell}\right)}-e^{\sigma\left(x+\xi_{j}-\xi_{\ell}\right)}\right] \sum_{i(\neq j)=1}^{k} U(x) U\left(x+\xi_{j}-\xi_{i}\right) d x\right| \\
\left.\leq 72 \sigma \int_{\xi_{\ell}-\xi_{i}}^{\infty}\left(|x|+\left|\xi_{j}-\xi_{\ell}\right|\right) \sum_{i(\neq j)=1}^{k} e^{-|x|} e^{-\left|x+\xi_{j}-\xi_{i}\right|} d x+\text { (h.o.t. }\right) \\
\leq 72 \sigma \sum_{i(\neq j)=1}^{k}\left\{\int_{\xi_{\ell}-\xi_{i}}^{\infty}|x| e^{-|x|} e^{-\left|x+\xi_{j}-\xi_{i}\right|} d x+\left|\xi_{j}-\xi_{\ell}\right| \int_{\xi_{\ell}-\xi_{i}}^{\infty} e^{-|x|} e^{-\left|x+\xi_{j}-\xi_{i}\right|} d x\right\} \\
\leq C \sigma^{2-\beta}(1+|j-\ell|)|\log \sigma|^{2}, \quad \text { for } j<\ell \text { and } j>\ell .
\end{aligned}
$$


Thus, we have

$$
\begin{gathered}
T_{\ell}^{c}(y) \triangleq \sum_{i(\neq j)=1}^{k} \int_{\mathbb{R}}\left[e^{-\sigma\left|\xi_{\ell}+y-x\right|}-e^{-\sigma\left|\xi_{\ell}-x\right|}\right] \sum_{i(\neq j)=1}^{k} U\left(x-\xi_{i}\right) U\left(x-\xi_{j}\right) d x \\
=\sum_{i(\neq j)=1}^{k} \tilde{C}(\sigma) e^{-\sigma\left|\xi_{\ell}-\xi_{j}\right|}\left[\sigma y \operatorname{sgn}\left(\xi_{j}-\xi_{\ell}\right)+O\left(\sigma^{2}|\log \sigma|^{2}\right)\right] \\
\quad+\sum_{i(\neq j)=1}^{k} C \sigma^{2-\beta}(1+|j-\ell|)|\log \sigma|^{2} \\
\leq \sum_{i(\neq j)=1}^{k} C(1+|\log \sigma|) \sigma^{1-\beta} e^{-\sigma\left|\xi_{\ell}-\xi_{j}\right|}\left[\sigma y \operatorname{sgn}\left(\xi_{j}-\xi_{\ell}\right)+O\left(\sigma^{2}|\log \sigma|^{2}\right)\right] \\
+C k(k-1) \sigma^{2-\beta}|\log \sigma|^{2} .
\end{gathered}
$$

Note that the first part $\sum_{i(\neq j)=1}^{k} \cdots$ in $(4.30)$ can be absorbed by the corresponding sum of the first part in (4.17) and in (4.18) for $j \neq \ell$.

For $j=\ell$ in $(4.23)$, we have

$$
\begin{aligned}
T_{\ell}(y) & \triangleq \int_{\mathbb{R}}\left[e^{-\sigma\left|\xi_{\ell}+y-x\right|}-e^{-\sigma\left|\xi_{\ell}-x\right|}\right] \sum_{i(\neq j)=1}^{k} U\left(x-\xi_{i}\right) U\left(x-\xi_{\ell}\right) d x \\
& =\int_{\mathbb{R}}\left[e^{-\sigma|y-x|}-e^{-\sigma|x|}\right] \sum_{i(\neq j)=1}^{k} U(x) U\left(x+\xi_{\ell}-\xi_{i}\right) d x \\
= & \left.-\sigma \int_{\mathbb{R}}[|y-x|-|x|] \sum_{i(\neq j)=1}^{k} U(x) U\left(x-\xi_{i}+\xi_{\ell}\right) d x+\text { (h.o.t. }\right)
\end{aligned}
$$

so that

$$
\begin{gathered}
\left|T_{\ell}(y)\right| \leq C \sigma|y| \sum_{i(\neq j)=1}^{k} \int_{\mathbb{R}} e^{-|x|} e^{-\left|x-\xi_{i}+\xi_{\ell}\right|} d x \\
\leq C \sigma|\log \sigma| \sum_{i(\neq j)=1}^{k}(1+|i-\ell||\log \sigma|) \sigma^{|i-\ell|(1-\beta)} \\
\leq C \sigma^{2-\beta}|\log \sigma|^{2}=O\left(\sigma^{2-\beta}|\log \sigma|^{2}\right), \text { for }|y| \leq \frac{1}{2}|\log \sigma| .
\end{gathered}
$$

Thus we have proved the following result.

LEMMA 4.2. For $\ell=1, \ldots, k$, and $|y| \leq \frac{1}{2}|\log \sigma|$, it holds that

$$
\begin{gathered}
V\left(\xi_{\ell}+y\right)-V\left(\xi_{\ell}\right)=\frac{1}{\omega}\left\{-\sigma \Psi(y)+O\left(\sigma^{2-\beta}|\log \sigma|^{2}\right)\right. \\
+\sum_{i(\neq j)=1}^{k} \hat{C}(\sigma) e^{-\sigma\left|\xi_{\ell}-\xi_{j}\right|}\left[\sigma y \operatorname{sgn}\left(\xi_{j}-\xi_{\ell}\right)+O\left(\sigma^{2}|\log \sigma|^{2}\right)\right] \\
\left.+O\left(\sigma^{2(1-\beta)}|\log \sigma|\right)+O\left(k^{2} \sigma^{2-\beta}|\log \sigma|^{2}\right)\right\},
\end{gathered}
$$


where $\hat{C}(\sigma)<40$ is shown in (4.12) and $\omega \geq 6 k$.

Proof. Substituting (4.17), (4.18), (4.19), (4.20), (4.30) and (4.31) into (4.9), we obtain (4.32) with the observations as follows,

$$
\sum_{j(\neq \ell)=1}^{k} O\left(\sigma^{2|j-\ell|(1-\beta)}(|j-\ell|+1)|\log \sigma|\right)=O\left(\sigma^{2(1-\beta)}|\log \sigma|\right)
$$

by using Lemma 1.3, and

$$
\begin{gathered}
C k(k-1) \sigma^{2-\beta}|\log \sigma|^{2}(\text { in }(4.30)) \\
+C \sigma^{2-\beta}|\log \sigma|^{2}(\text { in }(4.31))=O\left(k^{2} \sigma^{2-\beta}|\log \sigma|^{2}\right) .
\end{gathered}
$$

We shall note that

$$
\frac{1}{\omega} O\left(k^{2} \sigma^{2-\beta}|\log \sigma|^{2}\right)=O\left(\sigma^{2(1-\beta)}|\log \sigma|^{2}\right)
$$

III) As we said in the beginning of this section, the next is to make an estimate of the inner products

$$
\left\langle S[W], U^{\prime}\left(\cdot-\xi_{\ell}\right)\right\rangle, \quad \ell=1, \ldots, k,
$$

where $S[W]$ is defined by $(2.15)$. Indeed,

$$
\begin{aligned}
& (4.33) \quad\left\langle S[W], U^{\prime}\left(\cdot-\xi_{\ell}\right)\right\rangle=\int_{\mathbb{R}} S[W](x) U^{\prime}\left(x-\xi_{\ell}\right) d x \\
& =\int_{\mathbb{R}} \frac{1-V(x)}{V(x)} W^{2}(x) U^{\prime}\left(x-\xi_{\ell}\right) d x+\sum_{\substack{i, j \\
i \neq j}} \int_{\mathbb{R}} U\left(x-\xi_{i}\right) U\left(x-\xi_{j}\right) U^{\prime}\left(x-\xi_{\ell}\right) d x .
\end{aligned}
$$

The two parts on the right-hand side of (4.33) are denoted by $E_{1}$ and $E_{2}$, respectively. First we have

$$
\begin{aligned}
E_{1}=\int_{\mathbb{R}} \frac{1-V(x)}{V(x)} & U^{2}\left(x-\xi_{\ell}\right) U^{\prime}\left(x-\xi_{\ell}\right) d x \\
& +\sum_{\substack{i \neq j \text { or } \\
i=j \neq \ell}} \int_{\mathbb{R}} \frac{1-V(x)}{V(x)} U\left(x-\xi_{i}\right) U\left(x-\xi_{j}\right) U^{\prime}\left(x-\xi_{\ell}\right) d x .
\end{aligned}
$$

REMARK 3. In the following estimates, we shall use Lemmas 4.1 and 4.2 , the latter is valid on the compact interval

$$
I_{\xi}=\left[\xi_{1}-\frac{1}{2}|\log \sigma|, \xi_{k}+\frac{1}{2}|\log \sigma|\right]
$$

However, when we deal with the integrals over $\mathbb{R}$, we extend the utilization of Lemma 4.2 beyond the interval $I_{\xi}$, while the details of justification that the compensation is a small amount of higher order in $\sigma$ are omitted.

a) The first term in (4.34) is

$$
\begin{aligned}
& \int_{\mathbb{R}} \frac{1-V}{V} U^{2}\left(x-\xi_{\ell}\right) U^{\prime}\left(x-\xi_{\ell}\right) d x=\int_{\mathbb{R}} \frac{1-V\left(\xi_{\ell}+y\right)}{V\left(\xi_{\ell}+y\right)} U^{2}(y) U^{\prime}(y) d y \\
& =\int_{\mathbb{R}} \frac{1-V\left(\xi_{\ell}\right)}{V\left(\xi_{\ell}+y\right)} U^{2}(y) U^{\prime}(y) d y+\int_{\mathbb{R}} \frac{V\left(\xi_{\ell}\right)-V\left(\xi_{\ell}+y\right)}{V\left(\xi_{\ell}+y\right)} U^{2}(y) U^{\prime}(y) d y .
\end{aligned}
$$




$$
\begin{gathered}
\int_{\mathbb{R}} \frac{1-V\left(\xi_{\ell}\right)}{V\left(\xi_{\ell}+y\right)} U^{2}(y) U^{\prime}(y) d y=\int_{\mathbb{R}} \frac{1}{3} \frac{V\left(\xi_{\ell}\right)-1}{V^{2}\left(\xi_{\ell}+y\right)} V^{\prime}\left(\xi_{\ell}+y\right) U^{3}(y) d y \\
\leq \frac{1}{3} \int_{\mathbb{R}} \frac{O\left(\sigma^{1-\beta}|\log \sigma|\right) \sigma}{\left[V\left(\xi_{\ell}\right)+V\left(\xi_{\ell}+y\right)-V\left(\xi_{\ell}\right]^{2}\right.} U^{3}(y) d y\left(\text { since } V^{\prime}(x) \leq \sigma\right. \text { can be checked) } \\
=\int_{\mathbb{R}} \frac{O\left(\sigma^{2-\beta}|\log \sigma|\right) U^{3}(y)}{\left[1+O\left(\sigma^{1-\beta}|\log \sigma|\right)+O(\sigma|\log \sigma|)+O\left(\sigma^{2(1-\beta)}|\log \sigma|^{2}\right)\right]^{2}} d y \text { (from (4.32)) } \\
=O\left(\sigma^{2-\beta}|\log \sigma|\right) \int_{\mathbb{R}} U^{3}(y) d y=O\left(\sigma^{2-\beta}|\log \sigma|\right),
\end{gathered}
$$

and

$$
\begin{aligned}
& \int_{\mathbb{R}} \frac{V\left(\xi_{\ell}\right)-V\left(\xi_{\ell}+y\right)}{V\left(\xi_{\ell}+y\right)} U^{2}(y) U^{\prime}(y) d y \\
& \quad=\int_{\mathbb{R}} \frac{1}{1+O\left(\sigma^{1-\beta}|\log \sigma|\right)}\left\{\Pi_{1}(y)+\Pi_{2}(y)+\Pi_{3}(y)\right\} U^{2}(y) U^{\prime}(y) d y,
\end{aligned}
$$

in which, according to (4.32),

$$
\begin{aligned}
\Pi_{1}(y) & =-\frac{\sigma}{\omega} \int_{\mathbb{R}}[|y-x|-|x|]=-\frac{\sigma}{\omega} \Psi(y) \\
\Pi_{2}(y) & =\sum_{j(\neq \ell)=1}^{k} \hat{C}(\sigma) e^{-\sigma\left|\xi_{\ell}-\xi_{j}\right|}\left[\frac{\sigma}{\omega} y \operatorname{sgn}\left(\xi_{j}-\xi_{\ell}\right)+O\left(\sigma^{2+\beta}|\log \sigma|^{2}\right)\right] \\
\Pi_{3}(y) & =\frac{1}{\omega}\left[O\left(\sigma^{2(1-\beta)}|\log \sigma|\right)+O\left(k^{2} \sigma^{2-\beta}|\log \sigma|^{2}\right)\right] \\
& =O\left(\sigma^{2(1-\beta)}|\log \sigma|^{2}\right) .
\end{aligned}
$$

By the property of (4.22) of the function $\Psi$, we have

$$
\int_{\mathbb{R}} \Pi_{1}(y) U^{2}(y) U^{\prime}(y) d y=0
$$

so that

$$
\begin{aligned}
& \int_{\mathbb{R}} \frac{1}{1+O\left(\sigma^{1-\beta}|\log \sigma|\right)} \Pi_{1}(y) U^{2}(y) U^{\prime}(y) d y \\
= & \int_{\mathbb{R}}\left(\frac{1}{1+O\left(\sigma^{1-\beta}|\log \sigma|\right)}-1\right) \Pi_{1}(y) U^{2}(y) U^{\prime}(y) d y \\
= & O\left(\sigma^{1-\beta}|\log \sigma|\right) \int_{\mathbb{R}}\left|\Pi_{1}(y)\right| U^{2}(y)\left|U^{\prime}(y)\right| d y,
\end{aligned}
$$

where

$$
\int_{\mathbb{R}}\left|\Pi_{1}(y)\right| U^{2}(y)\left|U^{\prime}(y)\right| d y \leq \int_{\mathbb{R}} \sigma^{1+\beta}|y| \cdot 6^{3} e^{-3|y|} d y=6^{3}\left(\frac{2}{9}\right) \sigma^{1+\beta}=48 \sigma^{1+\beta} .
$$

Hence we have

$$
\int_{\mathbb{R}} \frac{1}{1+O\left(\sigma^{1-\beta}|\log \sigma|\right)} \Pi_{1}(y) U^{2}(y) U^{\prime}(y) d y=O\left(\sigma^{2}|\log \sigma|\right) .
$$


Next we get

$$
\begin{gathered}
\int_{\mathbb{R}} \frac{1}{1+O\left(\sigma^{1-\beta}|\log \sigma|\right)} \Pi_{2}(y) U^{2}(y) U^{\prime}(y) d y \\
=\sum_{j(\neq \ell)=1}^{k} \hat{C}(\sigma) e^{-\sigma\left|\xi_{\ell}-\xi_{j}\right|} \frac{\sigma}{\omega} \int_{\mathbb{R}} y \operatorname{sgn}\left(\xi_{j}-\xi_{\ell}\right) U^{2}(y) U^{\prime}(y) d y+O\left(\sigma^{2+\beta}|\log \sigma|^{2}\right) k \\
=\sum_{j(\neq \ell)=1}^{k} d(\sigma) e^{-\sigma\left|\xi_{\ell}-\xi_{j}\right|} \sigma^{1+\beta} \operatorname{sgn} \frac{\xi_{j}-\xi_{\ell}}{\left|\xi_{j}-\xi_{\ell}\right|}+O\left(\sigma^{2}|\log \sigma|^{2}\right),
\end{gathered}
$$

where the constant $d(\sigma)$, given by

$$
\begin{aligned}
d(\sigma) & =\hat{C}(\sigma) \frac{\sigma^{-\beta}}{\omega}\left(\frac{1}{3} \int_{\mathbb{R}} U^{3}(y) d y\right)=\hat{C}(\sigma) \frac{\sigma^{-\beta}}{\omega}\left(\frac{1}{3} \cdot \frac{36}{5}\right) \\
& =\frac{12}{5} \hat{C}(\sigma)\left(\frac{\sigma^{-\beta}}{\omega}\right),
\end{aligned}
$$

is uniformly bounded because $\hat{C}(\sigma)<40$ and $\left(\sigma^{-\beta} / \omega\right) \leq \sigma^{-\beta} /(6 k)=O(1)$.

Finally we have

$$
\int_{\mathbb{R}} \frac{1}{1+O\left(\sigma^{1-\beta}|\log \sigma|\right)} \Pi_{3}(y) U^{2}(y) U^{\prime}(y) d y=O\left(\sigma^{2(1-\beta)}|\log \sigma|^{2}\right) .
$$

From (4.36), (4.37), (4.39), (4.40) and (4.42) it follows that

$$
\begin{aligned}
& \int_{\mathbb{R}} \frac{1-V(x)}{V(x)} U^{2}\left(x-\xi_{\ell}\right) U^{\prime}\left(x-\xi_{\ell}\right) d x \\
& \quad=\sum_{j(\neq \ell)=1}^{k} d(\sigma) e^{-\sigma\left|\xi_{\ell}-\xi_{j}\right|} \sigma^{1+\beta} \operatorname{sgn}\left(\xi_{j}-\xi_{\ell}\right)+O\left(\sigma^{2(1-\beta)}|\log \sigma|^{2}\right)
\end{aligned}
$$

b) Now we estimate the $E_{2}$ part in (4.33) and the second part of $E_{1}$ in (4.34), both involve the triplet product $U\left(x-\xi_{i}\right) U\left(x-\xi_{j}\right) U^{\prime}\left(x-\xi_{\ell}\right)$. Note that

$$
\int_{\mathbb{R}} U\left(x-\xi_{i}\right) U\left(x-\xi_{j}\right) U^{\prime}\left(x-\xi_{\ell}\right) d x=O\left(\sigma^{2(1-\beta)}\right),
$$

if $\max \{|i-j|,|j-\ell|,|\ell-i|\} \geq 2$. Suppose $i=\ell$ (similarly if $j=\ell$ ). Then for $j<\ell$ we get

$$
\begin{gathered}
\int_{\mathbb{R}} U\left(x-\xi_{\ell}\right) U\left(x-\xi_{j}\right) U^{\prime}\left(x-\xi_{\ell}\right) d x=-\frac{1}{2} \int_{\mathbb{R}} U^{2}\left(x-\xi_{\ell}\right) U^{\prime}\left(x-\xi_{j}\right) d x \\
=-\frac{1}{2} \int_{\mathbb{R}} U^{2}(x) U^{\prime}\left(x-\xi_{j}+\xi_{\ell}\right) d x=3 \int_{\mathbb{R}} U^{2}(x) e^{-\left|x-\xi_{j}+\xi_{\ell}\right|} d x+Q_{1} \\
=3 \int_{\mathbb{R}} e^{-x} U^{2}(x) d x e^{-\left|\xi_{j}-\xi_{\ell}\right|}+Q_{1}+Q_{2}
\end{gathered}
$$

where

$$
\int_{\mathbb{R}} e^{-x} U^{2}(x) d x=12 \text {, by Lemma } 1.1
$$




$$
\begin{aligned}
\left|Q_{1}\right| & =\left|-\frac{1}{2} \int_{\mathbb{R}} U^{2}(x) U^{\prime}\left(x-\xi_{j}+\xi_{\ell}\right) d x-3 \int_{\mathbb{R}} U^{2}(x) e^{-\left|x-\xi_{j}+\xi_{\ell}\right|} d x\right| \\
& \leq\left|3 \int_{\xi_{j}-\xi_{\ell}}^{\infty} U^{2}(x) e^{-\left(x-\xi_{j}+\xi_{\ell}\right)}\left[\frac{1-e^{-\left(x-\xi_{j}+\xi_{\ell}\right)}}{\left(1+e^{-\left(x-\xi_{j}+\xi_{\ell}\right)}\right)^{3}}-1\right] d x\right| \\
& +\left|3 \int_{-\infty}^{\xi_{j}-\xi_{\ell}} U^{2}(x) e^{-\left(\xi_{j}-\xi_{\ell}-x\right)}\left[\frac{1-e^{-\left(\xi_{j}-\xi_{\ell}-x\right)}}{\left(1+e^{\left(\xi_{j}-\xi_{\ell}-x\right)}\right)^{3}}-1\right] d x\right| \\
& \leq 108 \int_{\mathbb{R}} e^{-2|x|} e^{-\left|x-\xi_{j}+\xi_{\ell}\right|}\left[4 e^{-\left|x-\xi_{j}+\xi_{\ell}\right|}+O\left(e^{-2\left|x-\xi_{j}+\xi_{\ell}\right|}\right)\right] d x \\
& \leq C \int_{\mathbb{R}} e^{-2|x|} e^{-2\left|x-\xi_{j}+\xi_{\ell}\right|} d x \quad(\text { by }(1.7)) \\
& \leq C e^{-2\left|\xi_{j}-\xi_{\ell}\right|}\left(1+\left|\xi_{j}-\xi_{\ell}\right|\right) \leq C \sigma^{2|j-\ell|(1-\beta)}(1+|j-\ell||\log \sigma|),
\end{aligned}
$$

and

$$
\begin{aligned}
\left|Q_{2}\right| & =\left|3 \int_{-\infty}^{\xi_{j}-\xi_{\ell}} U^{2}(x)\left[e^{-\left|x-\xi_{j}+\xi_{\ell}\right|}-e^{-\left(x-\xi_{j}+\xi_{\ell}\right)}\right] d x\right| \\
& \leq 108 \int_{-\infty}^{\xi_{j}-\xi_{\ell}} e^{2 x}\left|2\left(x-\xi_{j}+\xi_{\ell}\right)\right| d x+\text { (h.o.t.) } \\
& =54 e^{-2\left|\xi_{j}-\xi_{\ell}\right|}+\text { (h.o.t.) } \\
& \leq C \sigma^{2|j-\ell|(1-\beta)} .
\end{aligned}
$$

Thus we find that

$$
\begin{gathered}
\int_{\mathbb{R}} U\left(x-\xi_{\ell}\right) U\left(x-\xi_{j}\right) U^{\prime}\left(x-\xi_{\ell}\right) d x \\
= \begin{cases}36 e^{-\left|\xi_{j}-\xi_{\ell}\right|}+O\left(\sigma^{2|j-\ell|(1-\beta)}(1+|j-\ell||\log \sigma|)\right), & \text { if } j<\ell, \\
-36 e^{-\left|\xi_{j}-\xi_{\ell}\right|}+O\left(\sigma^{2|j-\ell|(1-\beta)}(1+|j-\ell||\log \sigma|)\right), & \text { if } j>\ell,\end{cases}
\end{gathered}
$$

We specify the sign difference between the case $j<\ell$, which we treated above, and the case $j>\ell$, for which the details are omitted. The difference is originated from different versions of $U^{\prime}(x)$ we use in two cases.

For $j<\ell$, the version $U^{\prime}(x)=-\frac{6 e^{-x}\left(1-e^{-x}\right)}{\left(1+e^{-x}\right)^{3}}$ is used. Then we count on the smallness of the integrals in $\left|Q_{2}\right|$ over $\left(-\infty, \xi_{j}-\xi_{\ell}\right] \subset \mathbb{R}^{-}$.

For $j>\ell$, another version $U^{\prime}(x)=\frac{6 e^{x}\left(1-e^{x}\right)}{\left(1+e^{x}\right)^{3}}$ is used. Then we can count on the smallness of the corresponding integral over $\left[\xi_{j}-\xi_{\ell}, \infty\right) \subset \mathbb{R}^{+}$.

The only other possibility for the triplet product terms will be

$$
\max \{|i-j|,|j-\ell|,|i-\ell|\} \geq 2,
$$

then (4.44) applies and the sum of all these terms will be of the order

$$
k O\left(\sigma^{2(1-\beta)} \sum_{\nu=1}^{\infty} \sigma^{\nu(1-\beta)}\right)=O\left(\sigma^{-\beta}\right) O\left(\sigma^{3(1-\beta)}\right)=O\left(\sigma^{3-4 \beta}\right) .
$$


Therefore, we obtain that, by Lemma 1.3 , and since $\beta<1 / 2$ implies $2(1-\beta)<$ $3-4 \beta$,

$$
\begin{aligned}
E_{2} & =\sum_{\substack{i, j \\
i \neq j}} \int_{\mathbb{R}} U\left(x-\xi_{i}\right) U\left(x-\xi_{j}\right) U^{\prime}\left(x-\xi_{\ell}\right) d x \\
& =36 \sum_{j(\neq \ell)=1}^{k} e^{-\left|\xi_{j}-\xi_{\ell}\right|} \operatorname{sgn} \frac{\xi_{\ell}-\xi_{j}}{\left|\xi_{\ell}-\xi_{j}\right|}+O\left(\sigma^{2(1-\beta)}|\log \sigma|\right)+O\left(\sigma^{3-4 \beta}\right) \\
& =36 \sum_{j(\neq \ell)=1}^{k} e^{-\left|\xi_{j}-\xi_{\ell}\right|} \operatorname{sgn} \frac{\xi_{\ell}-\xi_{j}}{\left|\xi_{\ell}-\xi_{j}\right|}+O\left(\sigma^{2(1-\beta)}|\log \sigma|\right) .
\end{aligned}
$$

Moreover, by Lemmas 4.1 and 4.2 , similar to (4.51), we have the estimate on the second part of $E_{1}$ in (4.34) as follows,

$$
\begin{gathered}
\sum_{\substack{i \neq j \text { or } \\
i=j \neq \ell}} \int_{\mathbb{R}} \frac{1-V}{V} U\left(x-\xi_{i}\right) U\left(x-\xi_{j}\right) U^{\prime}\left(x-\xi_{\ell}\right) d x \\
=O\left(\sigma^{1-\beta}|\log \sigma|\right)\left[6^{3} \sum_{j(\neq \ell)=1}^{k} e^{-\left|\xi_{j}-\xi_{\ell}\right|}+O\left(\sigma^{3-4 \beta}\right)\right] \\
=O\left(\sigma^{1-\beta}|\log \sigma|\right)\left[O\left(\sigma^{1-\beta}\right)+O\left(\sigma^{3-4 \beta}\right)\right] \\
=O\left(\sigma^{2(1-\beta)}|\log \sigma|\right) .
\end{gathered}
$$

With these preparations, we are now ready to prove a key result in the following theorem.

THEOREM 4.3. Under the same assumptions as in Theorem 3.6, it holds that

$$
\begin{array}{r}
\int_{\mathbb{R}} S[W](x) U^{\prime}\left(x-\xi_{\ell}\right) d x=\frac{\partial}{\partial \xi_{\ell}}\left[\sum_{j \neq \ell} F\left(\left|\xi_{j}-\xi_{\ell}\right|\right)\right]+O\left(\sigma^{2(1-\beta)}|\log \sigma|^{2}\right) \\
\ell=1, \ldots, k
\end{array}
$$

in which

$$
F(r)=-d(\sigma) \sigma^{1+\beta}|r|-36 e^{-|r|}
$$

where $d(\sigma)$ is a uniformly bounded, positive constant given by (4.41). Moreover, for any given $0<\mu<1$, there is a constant $C_{\mu}>0$ such that $S[W] \in L_{\mu}^{\infty}$ and

$$
\|S[W]\|_{\mu} \leq C_{\mu} \sigma^{1-\beta-\frac{\mu}{2}} .
$$

Proof. By substituting (4.43), (4.51) and (4.52) into (4.33), we can confirm that

$$
\begin{gathered}
\int_{\mathbb{R}} S[W](x) U^{\prime}\left(x-\xi_{\ell}\right) d x=\sum_{j \neq \ell} d(\sigma) e^{-\sigma\left|\xi_{j}-\xi_{\ell}\right|} \sigma^{1+\beta} \operatorname{sgn}\left(\xi_{j}-\xi_{\ell}\right) \\
+O\left(\sigma^{2(1-\beta)}|\log \sigma|^{2}\right)+\sum_{j \neq \ell} 36 e^{-\left|\xi_{j}-\xi_{\ell}\right|} \operatorname{sgn}\left(\xi_{\ell}-\xi_{j}\right) \\
+O\left(\sigma^{2(1-\beta)}|\log \sigma|\right) .
\end{gathered}
$$


Moreover, we see that $(4.57)$

$$
\begin{gathered}
\left|\sum_{j \neq \ell} d(\sigma)\left[e^{-\sigma\left|\xi_{j}-\xi_{\ell}\right|}-1\right] \sigma^{1+\beta} \operatorname{sgn}\left(\xi_{j}-\xi_{\ell}\right)\right| \\
\leq \sum_{j \neq \ell} d(\sigma)\left[\sigma\left|\xi_{j}-\xi_{\ell}\right|+O\left(\sigma^{2}\left|\xi_{j}-\xi_{\ell}\right|^{2}\right)\right] \sigma^{1+\beta}=O\left(\sigma^{2+\beta} k(k-1)|\log \sigma|\right) \\
=O\left(\sigma^{2-\beta}|\log \sigma|\right)
\end{gathered}
$$

which can be absorbed by the last term in (4.56), i.e., $O\left(\sigma^{2(1-\beta)}|\log \sigma|\right)$. Therefore, (4.56) yields

$$
\begin{gathered}
\int_{\mathbb{R}} S[W](u) U^{\prime}\left(x-\xi_{\ell}\right) d x=\sum_{j \neq \ell}\left[-d(\sigma) \sigma^{1+\beta} \operatorname{sgn}\left(\xi_{\ell}-\xi_{j}\right)+36 e^{-\left|\xi_{j}-\xi_{\ell}\right|} \operatorname{sgn}\left(\xi_{\ell}-\xi_{j}\right)\right] \\
\quad+O\left(\sigma^{2(1-\beta)}|\log \sigma|^{2}\right) \\
=\frac{\partial}{\partial \xi_{\ell}}\left[\sum_{j \neq \ell} F\left(\left|\xi_{j}-\xi_{\ell}\right|\right)\right]+O\left(\sigma^{2(1-\beta)}|\log \sigma|^{2}\right),
\end{gathered}
$$

which is $(4.53)$.

Now we prove the second statement of this theorem and (4.55). For each $x \in \mathbb{R}$, there is a point $\xi_{i_{0}}$ among $\xi_{1}, \ldots, \xi_{k}$ such that

$$
\left|x-\xi_{i_{0}}\right|=\min _{1 \leq i \leq k}\left|x-\xi_{i}\right| .
$$

Without loss of generality, we suppose that $x \in\left[\xi_{i_{0}}, \xi_{i_{0}+1}\right)$. It is possible that $\left|x-\xi_{i_{0}}\right|=\left|x-\xi_{i_{0}+1}\right|$. Then we have

$$
\begin{array}{r}
\left|\sum_{\substack{i, j \\
i \neq j}} U\left(x-\xi_{i}\right) U\left(x-\xi_{j}\right)\right| \leq \sum_{j \neq i_{0}} 36 e^{-\left|x-\xi_{i_{0}}\right|} e^{-\left|x-\xi_{j}\right|} \\
+\sum_{j \neq i_{0}+1} 36 e^{-\left|x-\xi_{j_{0}+1}\right|} e^{-\left|x-\xi_{j}\right|}+\sum_{i \neq i_{0}, i_{0}+1} \sum_{j \neq i} 36 e^{-\left|x-\xi_{i}\right|} e^{-\left|x-\xi_{j}\right|} .
\end{array}
$$

In the first sum, the largest term is

$$
\begin{gathered}
36 e^{-\left|x-\xi_{i_{0}}\right|} e^{-\left|x-\xi_{i_{0}+1}\right|}=36 e^{-\mu \min _{1 \leq i \leq k}\left|x-\xi_{i}\right|}\left[e^{-(1-\mu)\left|x-\xi_{i_{0}}\right|} e^{-\left|x-\xi_{i_{0}+1}\right|}\right] \\
=36 e^{-\mu \min _{1 \leq i \leq k}\left|x-\xi_{i}\right|}\left[e^{-(1-\mu)\left|x-\xi_{i_{0}}\right|} e^{-\left|\xi_{i_{0}+1}-\xi_{i_{0}}\right|+\left|x-\xi_{i_{0}}\right|}\right] \\
\leq 36 e^{-\mu \min _{1 \leq i \leq k}\left|x-\xi_{i}\right|}\left[\sigma^{1-\beta} e^{\mu\left|x-\xi_{i_{0}}\right|}\right] \\
\leq 36 e^{-\mu \min _{1 \leq i \leq k}\left|x-\xi_{i}\right|}\left[\sigma^{1-\beta} e^{\frac{\mu}{2}|\log \sigma|}\right] \\
=36 e^{-\mu \min _{1 \leq i \leq k}\left|x-\xi_{i}\right|}\left[\sigma^{1-\beta-\mu / 2}\right]
\end{gathered}
$$


which implies that

$$
\begin{gathered}
\sum_{j \neq i_{0}} 36 e^{-\left|x-\xi_{i_{0}}\right|} e^{-\left|x-\xi_{j}\right|} \leq 36 e^{-\mu \min _{1 \leq i \leq k}\left|x-\xi_{i}\right|}\left[\sigma^{1-\beta-\mu / 2}\right. \\
\left.+2 \sigma^{2(1-\beta)-\mu / 2}+2 \sigma^{3(1-\beta)-\mu / 2}+\cdots\right] \\
\leq C e^{-\mu \min _{1 \leq i \leq k}\left|x-\xi_{i}\right|} \sigma^{1-\beta-\mu / 2} .
\end{gathered}
$$

It is easy to see that the second sum $\sum_{j \neq i_{0}+1} \cdots$ satisfies the same estimate (4.60). Moreover,

$$
\begin{gathered}
36 \sum_{i \neq i_{0}, i_{0}+1}\left[e^{-\left|x-\xi_{i}\right|} \sum_{j \neq i} e^{-\left|x-\xi_{j}\right|}\right] \\
\leq 36\left[2 \sigma^{1-\beta}+\sigma^{2(1-\beta)}+\cdots\right]\left[2 e^{-\min _{1 \leq i \leq k}\left|x-\xi_{i}\right|}\left(1+\sigma^{1-\beta}+\sigma^{2(1-\beta)}+\cdots\right)\right] \\
\leq C e^{-\min _{1 \leq i \leq k}\left|x-\xi_{i}\right|} \sigma^{1-\beta} .
\end{gathered}
$$

Therefore, we have the following estimate for $x \in I_{\xi}$, where $I_{\xi}$ is the interval defined in (4.35).

1) If $x \in I_{\xi}$, then by (4.59), (4.60) and (4.61), with the observation

$$
\frac{1-V(x)}{V(x)}=O\left(\sigma^{1-\beta}|\log \sigma|\right),
$$

we have

$$
\begin{gathered}
S[W](x)=\frac{1-V(x)}{V(x)} W^{2}(x)+\sum_{\substack{i, j \\
i \neq j}} U\left(x-\xi_{i}\right) U\left(x-\xi_{j}\right) \\
=O\left(\sigma^{1-\beta}|\log \sigma|\right)\left[e^{-\min _{1 \leq i \leq k}\left|x-\xi_{i}\right|}\left(1+\sigma^{1-\beta}+\sigma^{2(1-\beta)}+\cdots\right)\right]^{2} \\
+e^{-\mu \min _{1 \leq i \leq k}\left|x-\xi_{i}\right|} O\left(\sigma^{1-\beta-\mu / 2}\right) \\
=e^{-\mu \min _{1 \leq i \leq k}\left|x-\xi_{i}\right|}\left[O\left(\sigma^{1-\beta}|\log \sigma|\right)+O\left(\sigma^{1-\beta-\mu / 2}\right)\right] \\
=e^{-\mu \min _{1 \leq i \leq k}\left|x-\xi_{i}\right|} O\left(\sigma^{1-\beta-\mu / 2}\right) .
\end{gathered}
$$

2) If $x \in \mathbb{R} \backslash I_{\xi}=\left(-\infty, \xi_{1}-\frac{1}{2}|\log \sigma|\right) \cup\left(\xi_{k}+\frac{1}{2}|\log \sigma|, \infty\right)$, then we can make an estimate of $S[W](x)$ as follows. Since $(2.12)$ implies $0<V(x) \leq 1$, we have

$$
|1-V(x)| \leq 1 \text {. }
$$

By the Lebesgue Dominated Convergence Theorem,

$$
\lim _{\sigma \rightarrow 0^{+}} V(x)=\frac{1}{\omega} \lim _{\sigma \rightarrow 0^{+}} \int_{\mathbb{R}} e^{-\sigma|z|} W^{2}(z+x) d z=1,
$$

which means for $\sigma>0$ sufficiently small, $V(x) \geq 1-\epsilon_{0}$ for some constant $\epsilon_{0}<1$. Thus

$$
\left|\frac{1-V(x)}{V(x)}\right| \leq \frac{1}{1-\epsilon_{0}}
$$


On the other hand, we have

$$
\begin{gathered}
\sum_{j=1}^{k} U^{2}\left(x-\xi_{j}\right) \leq 36 \sum_{j=1}^{k} e^{-2\left|x-\xi_{j}\right|} \quad\left(\text { since } x \in \mathbb{R} \backslash I_{\xi}\right) \\
\leq 36 e^{-\mu \min _{1 \leq i \leq k}\left|x-\xi_{i}\right|} e^{-(2-\mu) \frac{1}{2}|\log \sigma|}\left(1+\sigma^{2(1-\beta)}+\sigma^{4(1-\beta)}+\cdots\right) \\
\leq C e^{-\mu \min _{1 \leq i \leq k}\left|x-\xi_{i}\right|} \sigma^{1-\mu / 2},
\end{gathered}
$$

and

$$
\begin{aligned}
\sum_{\substack{i, j \\
i \neq j}} U\left(x-\xi_{i}\right) U\left(x-\xi_{j}\right) & \leq C e^{-\mu \min _{1 \leq i \leq k}\left|x-\xi_{i}\right|} \sigma^{\frac{1}{2}(1-\mu)} \sigma^{\frac{1}{2}+(1-\beta)} \\
& \leq C e^{-\mu \min _{1 \leq i \leq k}\left|x-\xi_{i}\right|} \sigma^{2-\beta-\mu / 2} .
\end{aligned}
$$

Hence we obtain the estimate

$$
\begin{aligned}
S[W](x) & \leq \frac{C}{1-\epsilon_{0}} e^{-\mu \min _{1 \leq i \leq k}\left|x-\xi_{i}\right|} \sigma^{1-\mu / 2}+C e^{-\mu \min _{1 \leq i \leq k}\left|x-\xi_{i}\right|} \sigma^{2-\beta-\mu / 2} \\
& =e^{-\mu \min _{1 \leq i \leq k}\left|x-\xi_{i}\right|} O\left(\sigma^{1-\mu / 2}\right) .
\end{aligned}
$$

In any case, (4.62) and (4.64) imply that (4.55) holds.

REMARK 4 (The second effect of $\mu$ ). In the previous remark in Section 3, we noted the effect of $\mu$ on the order of $k=O\left(\sigma^{-\beta}\right)$ in (3.63). Here is the second effect of the choice of the norm parameter $\mu$. From (4.55), we see that for $0<\mu \leq 1 / 3$ being chosen very close to zero, the upper bound of the $\mu$-norm of $S[W]$ will be very close to the order $\sigma^{1-\beta}$, i.e.,

$$
C_{\mu} \sigma^{1-\beta-\mu / 2} \approx C_{\mu} \sigma^{1-\beta}
$$

\section{Solution to the Augmented Nonlocal Problem}

In this section, we shall prove that the augmented nonlocal problem (2.19) admits a solution $u=W+\varphi_{\sigma, \xi}$ and $\varphi_{\sigma, \xi}$ turns out to be a small perturbation. Recall that problem (2.19) is equivalent to the problem (2.20), which we write again as follows

$$
\begin{aligned}
& L(\varphi)=S[W]+P(W, \varphi)+\sum_{j=1}^{k} \gamma_{j} W_{j} \\
& \left\langle\varphi, W_{j}\right\rangle=0, \quad j=1, \ldots, k \\
& \varphi(x) \rightarrow 0, \quad \text { as }|x| \rightarrow \infty
\end{aligned}
$$

where $L$ is the linear operator defined in (3.1), $S[W]$ is given in (2.15), and $P(W, \varphi)$ is given in (2.21).

By Theorem 4.3, for any $0<\mu \leq 1 / 3, S[W] \in L_{\mu}^{\infty}$ and

$$
\|S(W)\|_{\mu} \leq C_{\mu} \sigma^{1-\beta-\mu / 2} .
$$

We now consider the nonlinear term $P(W, \varphi)$. According to $(2.21)$,

$$
P(W, \varphi)=P_{1}(W, \varphi)+P_{2}(W, \varphi),
$$


where

(5.3)

$$
P_{1}(W, \varphi)=(W+\varphi)^{2}\left[\frac{1}{T\left[(W+\varphi)^{2}\right]}-\frac{1}{V}+\frac{2 T[W \varphi]}{V^{2}}\right]-(2 W+\varphi) \varphi \frac{2 T[W \varphi]}{V^{2}}
$$

and

$$
P_{2}(W, \varphi)=-2 W \varphi\left(1-\frac{1}{V}\right)+2 W^{2}\left[\frac{\langle W, \varphi\rangle}{\omega}-\frac{T[W \varphi]}{V^{2}}\right]+\frac{\varphi^{2}}{V}
$$

We have

$$
\begin{gathered}
T\left[(W+\varphi)^{2}\right]=V+2 T[W \varphi]+T\left[\varphi^{2}\right] \\
T[W \varphi]=\frac{1}{\omega} \int_{\mathbb{R}} W \varphi d x+\frac{1}{\omega} \int_{\mathbb{R}}\left(e^{-\sigma|x-y|}-1\right) W(y) \varphi(y) d y
\end{gathered}
$$

where

$$
\begin{gathered}
\frac{1}{\omega}\left|\int_{\mathbb{R}}\left(e^{-\sigma|x-y|}-1\right) W(y) \varphi(y) d y\right| \leq \frac{1}{6 k} \int_{\mathbb{R}} \sigma^{|x-y|} W(y)|\varphi(y)| d y \\
\leq \frac{\sigma\|\varphi\|_{\mu}}{6 k} \sum_{j=1}^{k} \int_{\mathbb{R}}|x-y| U\left(y-\xi_{j}\right) d y \\
\leq \frac{\sigma\|\varphi\|_{\mu}}{k} \sum_{j=1}^{k} \int_{\mathbb{R}}|x-y| e^{-\left|y-\xi_{j}\right|} d y \\
=\frac{\sigma\|\varphi\|_{\mu}}{k} \sum_{j=1}^{k} \int_{\mathbb{R}}|z| e^{-\left|x-\xi_{j}-z\right|} d z \\
=\frac{\sigma\|\varphi\|_{\mu}}{k} \sum_{j=1}^{k}\left(2 e^{-\left|x-\xi_{j}\right|}+2\left|x-\xi_{j}\right|\right) \\
\leq \frac{\sigma\|\varphi\|_{\mu}}{k}[O(1)+k(k+1)|\log \sigma|] \quad\left(\text { for any } x \in I_{\xi}\right) \\
=\sigma^{1+\beta}\|\varphi\|_{\mu}\left[O(1)+O\left(\sigma^{-2 \beta}|\log \sigma|\right)\right]=O\left(\sigma^{1-\beta}|\log \sigma|\right)\|\varphi\|_{\mu} .
\end{gathered}
$$

Hence, we obtain

$$
\begin{aligned}
T[W \varphi] & =O\left(\sigma^{\beta}\right)\|\varphi\|_{\mu} \int_{\mathbb{R}} W(x) d x+O\left(\sigma^{1-\beta}|\log \sigma|\right)\|\varphi\|_{\mu} \\
& =O\left(\sigma^{\beta}\right)\|\varphi\|_{\mu},
\end{aligned}
$$

and likewise, by using Lemma 1.5 , we can get

$$
T\left[\varphi^{2}\right]=O(1)\left\|\varphi^{2}\right\|_{\mu} .
$$

Then we obtain

$$
\begin{aligned}
\left|P_{1}(W, \varphi)\right| & \leq C\left(W^{2}+\varphi^{2}\right) T\left[\varphi^{2}\right]+C\left|2 W \varphi+\varphi^{2}\right||T[W \varphi]| \\
& \leq C e^{-2 \mu \min _{1 \leq i \leq k}\left|x-\xi_{i}\right|}\|\varphi\|_{\mu}^{2} .
\end{aligned}
$$

provided that $\|\varphi\|_{\mu}=O(1)$. Here we admit $x \in I_{\xi}$. 
On the other hand, we have

$$
\begin{gathered}
\left|P_{2}(W, \varphi)\right| \leq 2 W|\varphi| O\left(\sigma^{1-\beta}|\log \sigma|\right)+2 W^{2} O\left(\sigma^{1-\beta}|\log \sigma|\right) O\left(\sigma^{\beta}\right)\|\varphi\|_{\mu}+C|\varphi|^{2} \\
\leq e^{-2 \mu \min _{1 \leq i \leq k}\left|x-\xi_{i}\right|}\left[\frac{12}{1-\sigma^{1-\beta}} O\left(\sigma^{1-\beta}|\log \sigma|\right)\|\varphi\|_{\mu}\right. \\
\left.\quad+\frac{72}{\left(1-\sigma^{1-\beta}\right)^{2}} O(\sigma|\log \sigma|)\|\varphi\|_{\mu}+C\|\varphi\|_{\mu}^{2}\right] \\
\leq C e^{-2 \mu \min _{1 \leq i \leq k}\left|x-\xi_{i}\right|}\left[\|\varphi\|_{\mu}^{2}+O\left(\sigma^{1-\beta}|\log \sigma|\right)\|\varphi\|_{\mu}\right] .
\end{gathered}
$$

Thus we have shown that in the range of $x \in I_{\xi}=\left[\xi_{1}-\frac{1}{2}|\log \sigma|, \xi_{k}+\frac{1}{2}|\log \sigma|\right]$, there exists a constant $C>0$ such that

$$
\|P(W, \varphi)\|_{\mu} \leq C\left[\|\varphi\|_{\mu}+O\left(\sigma^{1-\beta}|\log \sigma|\right)\right]\|\varphi\|_{\mu}
$$

provided that $\|\varphi\|_{\mu}=O(1)$.

Next we consider the range $x \in \mathbb{R} \backslash I_{\xi}$. In that case,

$$
\begin{gathered}
|P(W, \varphi)|=\left|\frac{(W+\varphi)^{2}}{T\left[(W+\varphi)^{2}\right]}-\frac{W^{2}}{V}-2 W \varphi+\frac{2 W^{2}}{\omega}\langle W, \varphi\rangle\right| \\
\leq\left|\frac{2 W \varphi+\varphi^{2}}{V}-2 W \varphi+\frac{2 W^{2}}{\omega}\langle W, \varphi\rangle\right|+C(W+\varphi)^{2}\left|2 T[W \varphi]+T\left[\varphi^{2}\right]\right| \\
=\left|\hat{P}_{1}(W, \varphi)\right|+\left|\hat{P}_{2}(W, \varphi)\right|,
\end{gathered}
$$

where

$$
\begin{gathered}
\left|\hat{P}_{1}(W, \varphi)\right| \leq C\left(\sum_{j=1}^{k} e^{-\left|x-\xi_{j}\right|}\right) e^{-\mu \min _{1 \leq i \leq k}\left|x-\xi_{i}\right|}\|\varphi\|_{\mu}+C e^{-2 \mu \min _{1 \leq i \leq k}\left|x-\xi_{i}\right|}\|\varphi\|_{\mu}^{2} \\
+\frac{72}{6 k}\left(\sum_{j=1}^{k} e^{-\left|x-\xi_{j}\right|}\right)^{2} \int_{\mathbb{R}} \sum_{j=1}^{k} e^{-\left|y-\xi_{j}\right|} e^{-\mu \min _{1 \leq i \leq k}\left|y-\xi_{i}\right|} d y\|\varphi\|_{\mu} \\
\leq C e^{-\mu \min _{1 \leq i \leq k}\left|x-\xi_{i}\right|}\left(\sigma^{1 / 2}\|\varphi\|_{\mu}+\|\varphi\|_{\mu}^{2}\right) \\
\leq C e^{-\mu \min _{1 \leq i \leq k}\left|x-\xi_{i}\right|}\left(\sigma^{1 / 2}\|\varphi\|_{\mu}+\|\varphi\|_{\mu}^{2}\right)
\end{gathered}
$$

and

$$
\begin{gathered}
\left|\hat{P}_{2}(W, \varphi)\right|=C(W+\varphi)^{2}\left|2 T[W \varphi]+T\left[\varphi^{2}\right]\right| \\
\leq C\left[\sum_{j=1}^{k} e^{-\left|x-\xi_{j}\right|}+e^{-\mu \min _{1 \leq i \leq k}\left|x-\xi_{i}\right|}\|\varphi\|_{\mu}\right]^{2}\left|2 T[W \varphi]+T\left[\varphi^{2}\right]\right| \\
\leq C e^{-\mu \min _{1 \leq i \leq k}\left|x-\xi_{i}\right|}\left(\sigma^{\frac{1}{2}(1-\mu)}+\|\varphi\|_{\mu}\right)\left(\sigma^{\frac{1}{2}}+\|\varphi\|_{\mu}\right) \\
\cdot \frac{C}{k} e^{-\sigma \min _{1 \leq i \leq k}\left|x-\xi_{i}\right|}\left[\int_{\mathbb{R}} W(y) d y\|\varphi\|_{\mu}+\int_{\mathbb{R}} e^{-\mu \min _{1 \leq i \leq k}\left|x-\xi_{i}\right|} d y\|\varphi\|_{\mu}^{2}\right]
\end{gathered}
$$


in which, by Lemma 1.5, we have

so that

$$
\frac{1}{k} \int_{\mathbb{R}} W(y) d y=6 \text { and } \frac{1}{k} \int_{\mathbb{R}} e^{-\mu \min _{1 \leq i \leq k}\left|y-\xi_{i}\right|} d y=O(1),
$$

$$
\left|\hat{P}_{2}(W, \varphi)\right| \leq C e^{-\mu \min _{1 \leq i \leq k}\left|x-\xi_{i}\right|}\left(\sigma^{1-\frac{\mu}{2}}\|\varphi\|_{\mu}+\|\varphi\|_{\mu}^{2}\right),
$$

provided that $\|\varphi\|_{\mu}=O(1)$. From (5.11) through (5.13) we get

$$
|P(W, \varphi)| \leq C e^{-\mu \min _{1 \leq i \leq k}\left|x-\xi_{i}\right|}\left(\sigma^{\frac{1}{2}}\|\varphi\|_{\mu}+\|\varphi\|_{\mu}^{2}\right),
$$

$$
\text { for } x \in \mathbb{R} \backslash I_{\xi} \text {. }
$$

LEMma 5.1. Assume that for $0<\mu \leq 1 / 3,\|\varphi\|_{\mu}=O(1)$ as $\sigma$ being sufficiently small. Then $P(W, \varphi)$ given by (2.21) satisfies the estimate

$$
\| P\left(W, \varphi\left\|_{\mu} \leq C\left[\|\varphi\|_{\mu}+O\left(\sigma^{1-\beta}|\log \sigma|\right)+O\left(\sigma^{\frac{1}{2}}\right)\right]\right\| \varphi \|_{\mu} .\right.
$$

Proof. Simply combine (5.10) for $x \in I_{\xi}$ and (5.14) for $x \in \mathbb{R} \backslash I_{\xi}$ to reach the conclusion.

The following is a key result on the solution to the augmented nonlocal problem (5.1). Its proof is based on the investigation of the principal approximation linear operator $L$, which we presented in Theorem 3.6, and on the prepared nonlinear estimates shown in Theorem 4.3 and Lemma 5.1.

THEOREM 5.2. There exists a constant $\check{\sigma}>0$ such that for $0<\sigma \leq \check{\sigma}$, under the assumption

$$
k=C \sigma^{-\beta} \text { and } 0<\beta<\frac{1-\mu}{2-\mu} \quad\left(\text { with } 0<\mu \leq \frac{1}{3}\right),
$$

for any given $\xi=\left(\xi_{1}, \ldots, \xi_{k}\right) \in \Lambda$ in (1.16), there exists a unique solution

$$
\varphi_{\sigma, \xi} \in L_{\mu}^{\infty}, \quad \gamma=\left(\gamma_{1}, \ldots, \gamma_{k}\right) \in \mathbb{R}^{k}
$$

of the augmented nonlocal problem:

$$
\begin{gathered}
S[W+\varphi](x)=\sum_{j=1}^{k} \gamma_{j} U^{\prime}\left(x-\xi_{j}\right), \quad x \in \mathbb{R}, \\
\int_{\mathbb{R}} \varphi(x) U^{\prime}\left(x-\xi_{j}\right) d x=0, \quad j=1, \ldots, k \\
\varphi(x) \rightarrow 0, \text { as }|x| \rightarrow \infty .
\end{gathered}
$$

Moreover,

$$
\left\|\varphi_{\sigma, \xi}\right\|_{\mu} \leq 2 \Gamma_{0} C_{\mu} \sigma^{1-\beta-\mu / 2}
$$

where $\Gamma_{0}$ is the constant in (3.80) and $C_{\mu}$ is the constant in (4.55).

ProOF. By the equivalence of problem (5.16) (aka (2.19)) and problem (5.1) (aka $(2.20)$ ), it suffices to show that problem (5.1) has a solution $\left(\varphi_{\sigma, \xi}, \gamma\right)$ as stated in this theorem.

According to Theorem 3.6, if we set

$$
h=S[W]+P(W, \varphi)
$$


then problem (5.1) is to find a solution $\varphi \in L_{\mu}^{\infty}$ of the equation

$$
\varphi=\mathcal{J}(h)=\mathcal{J}(S[W]+P(W, \varphi))
$$

and then $\gamma=\Pi(h)$ can be determined, where the bounded linear operator $\mathcal{J}$ on $L_{\mu}^{\infty}$ is well-defined and (3.80) holds. Define

$$
Q(\varphi) \triangleq \mathcal{J}(S[W]+P(W, \varphi)), \quad \text { for } \varphi \in L_{\mu}^{\infty} .
$$

Then problem (5.1) is reduced to finding a fixed point $\varphi \in L_{\mu}^{\infty}$ of the mapping $Q$, i.e.,

$$
\varphi=Q(\varphi)
$$

Let $B$ be the closed, convex set in $L_{\mu}^{\infty}$ defined by

$$
B=\left\{\begin{array}{ll} 
& \|\varphi\|_{\mu} \leq 2 \Gamma_{0} C_{\mu} \sigma^{1-\beta-\mu / 2} \\
\varphi \in L_{\mu}^{\infty}: & \left\langle\varphi, W_{j}\right\rangle=0, \quad j=1, \ldots, k, \\
\varphi(x) \rightarrow 0 \text { as }|x| \rightarrow \infty
\end{array}\right\} .
$$

We use the contraction mapping argument to prove that $Q$ has a fixed point $\varphi_{\sigma, \xi}$ in $B$.

First we show $Q(B) \subset B$. For any $\varphi \in B$, by the description of problem (3.13) and the definition of $\mathcal{J}, Q(\varphi)$ satisfies automatically the two conditions in (5.21),

$$
\left\langle Q(\varphi), W_{j}\right\rangle=0, j=1, \ldots, k, \text { and } Q(\varphi)(x) \rightarrow 0 \text { as }|x| \rightarrow \infty .
$$

By Theorem 4.3 and Lemma 5.1, there is a small constant $\check{\sigma}>0$, such that for $0<\sigma \leq \check{\sigma}$

$$
\begin{aligned}
\|Q(\varphi)\|_{\mu} & \leq\|\mathcal{J}\|_{\mathcal{L}\left(L_{\mu}^{\infty}\right)}\left\{\|S(W)\|_{\mu}+\|P(W, \varphi)\|_{\mu}\right\} \\
& \leq \Gamma_{0}\left\{C_{\mu} \sigma^{1-\beta-\mu / 2}+C\left[\|\varphi\|_{\mu}+O\left(\sigma^{1-\beta}|\log \sigma|\right)+O\left(\sigma^{\frac{1}{2}}\right)\right]\|\varphi\|_{\mu}\right\} \\
& \leq 2 \Gamma_{0} C_{\mu} \sigma^{1-\beta-\mu / 2}, \text { for any } \varphi \in B .
\end{aligned}
$$

Indeed, $\check{\sigma}>0$ can be chosen to ensure that for $0<\sigma \leq \check{\sigma}$, in (5.22),

$$
C\left[2 \Gamma_{0} C_{\mu} \sigma^{1-\beta-\frac{\mu}{2}}+O\left(\sigma^{1-\beta}|\log \sigma|\right)+O\left(\sigma^{\frac{1}{2}}\right)\right] \leq \frac{1}{8},
$$

so that (5.22) holds. This implies $Q(B) \subset B$, for $0<\sigma \leq \check{\sigma}$.

Next, for any $\varphi_{1}, \varphi_{2} \in B$, we can estimate

$$
\begin{gathered}
\left\|Q\left(\varphi_{1}\right)-Q\left(\varphi_{2}\right)\right\| \leq\|\mathcal{J}\|\left\|P\left(W, \varphi_{1}\right)-P\left(W, \varphi_{2}\right)\right\|_{\mu} \\
\leq \Gamma_{0}\left\{\left\|P_{1}\left(W, \varphi_{1}\right)-P_{1}\left(W, \varphi_{2}\right)\right\|_{\mu}+\left\|P_{2}\left(W, \varphi_{1}\right)-P_{2}\left(W, \varphi_{2}\right)\right\|_{\mu}\right\}
\end{gathered}
$$

through the similar steps as we have gone in (5.5) through (5.10), for $x \in I_{\xi}$. Then a corresponding estimate can be made by the similar steps as (5.11) through (5.14), for $x \in \mathbb{R} \backslash I_{\xi}$. The details are omitted here. We can conclude that for $0<\sigma \leq \check{\sigma}$, where $\check{\sigma}>0$ is the constant chosen in accordance with (5.23), it holds that

$$
\left\|Q\left(\varphi_{1}\right)-Q\left(\varphi_{2}\right)\right\|_{\mu} \leq \frac{1}{2}\left\|\varphi_{1}-\varphi_{2}\right\|_{\mu}, \text { for any } \varphi_{1}, \varphi_{2} \in B
$$

Therefore, $Q$ is a contraction mapping on the set $B$.

By the Schauder fixed point theorem, there exists a unique fixed point $\varphi=\varphi_{\sigma, \xi}$ of $Q$ in the set $B$, which is a solution of problem (5.1) and of the original problem (5.16), and $\gamma=\gamma_{\sigma, \xi}$ can be determined by solving (3.16). The property (5.17) follows from (5.21). 
Let $\varphi_{\sigma, \xi}$ be the solution shown in Theorem 5.2. In order to prepare for the final stage, we need to know about

$$
\begin{aligned}
\int_{\mathbb{R}} S\left[W+\varphi_{\sigma, \xi}\right](x) U^{\prime}( & \left.x-\xi_{\ell}\right) d x \\
= & \int_{\mathbb{R}} S[W](x) U^{\prime}\left(x-\xi_{\ell}\right) d x \\
& \quad+\int_{\mathbb{R}}\left[S\left[W+\varphi_{\sigma, \xi}\right](x)-S[W](x)\right] U^{\prime}\left(x-\xi_{\ell}\right) d x,
\end{aligned}
$$

for $\ell=1, \ldots, k$. The information about $\int_{\mathbb{R}} S[W](x) U^{\prime}\left(x-\xi_{\ell}\right) d x$ is known in Theorem 4.3. Here we study the second part. Denote by $S^{\prime}$ the Fréchet derivative of the operator $S$. We have

$$
\begin{gathered}
\int_{\mathbb{R}}\left[S\left[W+\varphi_{\sigma, \xi}\right](x)-S[W](x)\right] U^{\prime}\left(x-\xi_{\ell}\right) d x \\
=\int_{\mathbb{R}} S^{\prime}[W]\left(\varphi_{\sigma, \xi}\right) U^{\prime}\left(x-\xi_{\ell}\right) d x+O\left(\sigma^{2(1-\beta-\mu / 2)}\right) \\
=\int_{\mathbb{R}}\left[\varphi_{\sigma, \xi}^{\prime \prime}-\varphi_{\sigma, \xi}+\frac{2 W \varphi_{\sigma, \xi}}{V}-\frac{2 W^{2}}{V^{2}} T\left[W \varphi_{\sigma, \xi}\right]\right] U^{\prime}\left(x-\xi_{\ell}\right) d x+O\left(\sigma^{2(1-\beta-\mu / 2)}\right),
\end{gathered}
$$

and according to Lemma 1.4, by integration by parts, we have

$$
\begin{gathered}
\int_{\mathbb{R}}\left[\varphi_{\sigma, \xi}^{\prime \prime}-\varphi_{\sigma, \xi}+2 U\left(x-\xi_{\ell}\right) \varphi_{\sigma, \xi}\right] U^{\prime}\left(x-\xi_{\ell}\right) d x \\
=\int_{\mathbb{R}} \varphi_{\sigma, \xi}\left[U^{\prime \prime \prime}\left(x-\xi_{\ell}\right)-U^{\prime}\left(x-\xi_{\ell}\right)+2 U\left(x-\xi_{\ell}\right) U^{\prime}\left(x-\xi_{\ell}\right)\right] d x=0 .
\end{gathered}
$$

Hence we get

$$
\begin{gathered}
\int_{\mathbb{R}}\left[S\left[W+\varphi_{\sigma, \xi}\right]-S[W]\right] U^{\prime}\left(x-\xi_{\ell}\right) d x-O\left(\sigma^{2(1-\beta-\mu / 2)}\right)(\text { in }(5.27)) \\
=\int_{\mathbb{R}}\left(\frac{2 W(x)}{V(x)}-2 U\left(x-\xi_{\ell}\right)\right) \varphi_{\sigma, \xi} U^{\prime}\left(x-\xi_{\ell}\right) d x \\
-\int_{\mathbb{R}} \frac{2 W^{2}}{V^{2}} T\left[W \varphi_{\sigma, \xi}\right] U^{\prime}\left(x-\xi_{\ell}\right) d x \\
=g_{1}\left(W, \varphi_{\sigma, \xi}, \ell\right)+g_{2}\left(W, \varphi_{\sigma, \xi}, \ell\right)
\end{gathered}
$$


where

$$
\begin{gathered}
\left|g_{1}\left(W, \varphi_{\sigma, \xi}, \ell\right)\right| \leq 2 \int_{\mathbb{R}}\left|W\left(\frac{1}{V}-1\right) \varphi_{\sigma, \xi}(x) U^{\prime}\left(x-\xi_{\ell}\right)\right| d x \\
+2 \sum_{j \neq \ell} \int_{\mathbb{R}} U\left(x-\xi_{j}\right)\left|\varphi_{\sigma, \xi}(x)\right|\left|U^{\prime}\left(x-\xi_{\ell}\right)\right| d x \\
\leq O\left(\sigma^{1-\beta}|\log \sigma|\right) \int_{\mathbb{R}} \sum_{j=1}^{k} e^{-\left|x-\xi_{j}\right|} e^{-\left|x-\xi_{\ell}\right|} e^{-\mu \min _{1 \leq i \leq k}\left|x-\xi_{i}\right|} d x\left\|\varphi_{\sigma, \xi}\right\|_{\mu} \\
+72 \sum_{j \neq \ell}^{k} \int_{\mathbb{R}} e^{-|x|} e^{-\left|x+\xi_{j}-\xi_{\ell}\right|} e^{-\mu \min _{1 \leq i \leq k}\left|x-\xi_{i}\right|} d x\left\|\varphi_{\sigma, \xi}\right\|_{\mu} \\
\leq O\left(\sigma^{1-\beta}|\log \sigma|\right) \sum_{j=1}^{k} \int_{\mathbb{R}} e^{-|x|} e^{-\left|x+\xi_{j}-\xi_{\ell}\right|} d x\left\|\varphi_{\sigma, \xi}\right\|_{\mu} \\
+72 \sum_{j \neq \ell} \int_{\mathbb{R}} e^{-|x|} e^{-\left|x+\xi_{j}-\xi_{\ell}\right|} d x\left\|\varphi_{\sigma, \xi}\right\|_{\mu} \\
\leq O\left(\sigma^{1-\beta}|\log \sigma|\right) O(1) 2 \Gamma_{0} C_{\mu} \sigma^{1-\beta-\mu / 2} \\
+72 O\left(\sigma^{1-\beta}|\log \sigma|\right) 2 \Gamma_{0} C_{\mu} \sigma^{1-\beta-\mu / 2} \\
=O\left(\sigma^{2(1-\beta)-\mu / 2}|\log \sigma|\right)+O\left(\sigma^{2(1-\beta)-\mu / 2}|\log \sigma|\right) \\
=O\left(\sigma^{2(1-\beta)-\mu / 2}|\log \sigma|\right)
\end{gathered}
$$

in which Lemmas 1.2 and 1.3 are used to claim

$$
\sum_{j=1}^{k} \int_{\mathbb{R}} e^{-|x|} e^{-\left|x+\xi_{j}-\xi_{\ell}\right|} d x=O(1)
$$

and

$$
\sum_{j \neq \ell} \int_{\mathbb{R}} e^{-|x|} e^{-\left|x+\xi_{j}-\xi_{\ell}\right|} d x=O\left(\sigma^{1-\beta}|\log \sigma|\right)
$$

Next, we have

$$
\begin{gathered}
g_{2}\left(W, \varphi_{\sigma, \xi}, \ell\right)=-\int_{\mathbb{R}} \frac{2 W^{2}}{V^{2}} T\left[W \varphi_{\sigma, \xi}\right] U^{\prime}\left(x-\xi_{\ell}\right) d x \\
=-\int_{\mathbb{R}} 2 U^{2}\left(x-\xi_{\ell}\right) U^{\prime}\left(x-\xi_{\ell}\right) T\left[W \varphi_{\sigma, \xi}\right](x) d x \\
-\sum_{\substack{i, j \\
\text { not both } \ell}} \int_{\mathbb{R}} 2 U\left(x-\xi_{i}\right) U\left(x-\xi_{j}\right) U^{\prime}\left(x-\xi_{\ell}\right) T\left[W \varphi_{\sigma, \xi}\right](x) d x+\text { Rmd }
\end{gathered}
$$

where the remainder

$$
\mathrm{Rmd}=\int_{\mathbb{R}} 2 W^{2}\left(1-\frac{1}{V^{2}}\right) T\left[W \varphi_{\sigma, \xi}\right] U^{\prime}\left(x-\xi_{\ell}\right) d x
$$


is a small amount of higher order in comparison with the first two parts on the right-hand side of (5.30). Note that

$$
\begin{gathered}
\frac{d}{d x} T\left[W \varphi_{\sigma, \xi}\right](x)=\frac{1}{\omega} \frac{d}{d x} \int_{\mathbb{R}} e^{-\sigma|x-y|} W(y) \varphi_{\sigma, \xi}(y) d y \\
=\frac{\sigma}{\omega}\left[\int_{x}^{\infty} e^{-\sigma|x-y|} W(y) \varphi_{\sigma, \xi}(y) d y-\int_{-\infty}^{x} e^{-\sigma|x-y|} W(y) \varphi_{\sigma, \xi}(y) d y\right] .
\end{gathered}
$$

Consequently, we have

$$
\begin{gathered}
\left|\frac{d}{d x} T\left[W \varphi_{\sigma, \xi}\right](x)\right| \leq C \sigma^{1+\beta}\left\|\varphi_{\sigma, \xi}\right\|_{\mu} \int_{-\infty}^{\infty} e^{-\sigma|x-y|} \sum_{j=1}^{k} e^{-\left|y-\xi_{j}\right|} e^{-\mu \min _{1 \leq i \leq k}\left|x-\xi_{i}\right|} d y \\
\leq C \sigma^{1+\beta} \sum_{j=1}^{k}\left[e^{-\sigma\left|x-\xi_{j}\right|}\left(\int_{\mathbb{R}} e^{-(1-\sigma)\left|y-\xi_{j}\right|} d y\right)\right]\left\|\varphi_{\sigma, \xi}\right\|_{\mu} \\
\leq C \sigma^{1+\beta} O\left(\sigma^{-\beta}\right)\left\|\varphi_{\sigma, \xi}\right\|_{\mu} \leq C \sigma^{1+\beta} O\left(\sigma^{-\beta}\right) 2 \Gamma_{0} C_{\mu} \sigma^{1-\beta-\mu / 2} \\
=O\left(\sigma^{2-\beta-\mu / 2}\right) .
\end{gathered}
$$

Hence, we have

$$
\begin{gathered}
\left|-\int_{\mathbb{R}} 2 U^{2}\left(x-\xi_{\ell}\right) U^{\prime}\left(x-\xi_{\ell}\right) T\left[W \varphi_{\sigma, \xi}\right](x) d x\right| \\
=\left|\frac{2}{3} \int_{\mathbb{R}} U^{3}\left(x-\xi_{\ell}\right) T^{\prime}\left[W \varphi_{\sigma, \xi}\right](x) d x\right| \leq C \int_{\mathbb{R}} e^{-3\left|x-\xi_{\ell}\right|}\left|T^{\prime}\left[W \varphi_{\sigma, \xi}\right](x)\right| d x \\
=O\left(\sigma^{2-\beta-\mu / 2}\right) .
\end{gathered}
$$

On the other hand, since it is shown that $T\left[W \varphi_{\sigma, \xi}\right]=O\left(\sigma^{\beta}\left\|\varphi_{\sigma, \xi}\right\|_{\mu}\right)$ in (5.6), which is valid for $x \in I_{\xi}$ or $x \in \mathbb{R} \backslash I_{\xi}$, but the details for $x \in \mathbb{R} \backslash I_{\xi}$ are omitted, here we can get

$$
\begin{aligned}
& \left|-\sum_{\substack{i, j \\
\text { not both } \ell}} \int_{\mathbb{R}} 2 U\left(x-\xi_{i}\right) U\left(x-\xi_{j}\right) U^{\prime}\left(x-\xi_{\ell}\right) T\left[W \varphi_{\sigma, \xi}\right](x) d x\right| \\
& \leq O\left(\sigma^{\beta}\left\|\varphi_{\sigma, \xi}\right\|_{\mu}\right) \sum_{\substack{i, j \\
\text { not both } \ell}} \int_{\mathbb{R}} e^{-\left|x-\xi_{i}\right|} e^{-\left|x-\xi_{j}\right|} e^{-\left|x-\xi_{\ell}\right|} d x \\
& \leq O\left(\sigma^{\beta}\left\|\varphi_{\sigma, \xi}\right\|_{\mu}\right) \sum_{j=1}^{k} \sum_{i(\neq \ell)=1}^{k} \int_{\mathbb{R}} e^{-|x|} e^{-\left|x+\xi_{i}-\xi_{j}\right|} e^{-\left|x+\xi_{i}-\xi_{\ell}\right|} d x \\
& \leq O\left(\sigma^{\beta}\left\|\varphi_{\sigma, \xi}\right\|_{\mu}\right) \sum_{j=1}^{k} \sum_{i(\neq \ell)=1}^{k} e^{-\left|\xi_{i}-\xi_{\ell}\right|} \int_{\mathbb{R}} e^{-\left|x+\xi_{i}-\xi_{j}\right|} d x \\
& \leq O\left(\sigma^{\beta}\left\|\varphi_{\sigma, \xi}\right\|_{\mu}\right) 2 k C \sigma^{1-\beta}=O\left(\sigma^{\beta} \sigma^{1-\beta-\mu / 2}\right) O\left(\sigma^{-\beta}\right) O\left(\sigma^{1-\beta}\right) \\
& \leq O\left(\sigma^{2(1-\beta)-\mu / 2}\right) .
\end{aligned}
$$


Substituting (5.29), (5.32) and (5.33) into (5.28), we reach the following estimate, which is stated as a lemma. Here $O\left(\sigma^{2(1-\beta)-\mu / 2}|\log \sigma|\right)$ is dominated by $O\left(\sigma^{2(1-\beta)-\mu}\right)$.

LEMMA 5.3. Under the same assumption as in Theorem 5.2, it holds that

$$
\int_{\mathbb{R}}\left(S\left[W+\varphi_{\sigma, \xi}\right]-S[W]\right) U^{\prime}\left(x-\xi_{\ell}\right) d x=O\left(\sigma^{2(1-\beta)-\mu}\right) .
$$

Now we prove the following key result.

THEOREM 5.4. Under the same assumption as in Theorem 5.2, for $\sigma$ sufficiently small, $\xi \in \Lambda$, let $\left(\varphi_{\sigma, \xi}, \gamma_{\sigma, \xi}\right) \in L_{\mu}^{\infty} \times \mathbb{R}^{k}$, where $\gamma_{\sigma, \xi}=\left(\gamma_{1}, \ldots, \gamma_{k}\right)$, be the solution of problem (5.16). Then, for $\ell=1, \ldots, k$,

$$
\begin{gathered}
\sum_{j=1}^{k} \gamma_{j} \int_{\mathbb{R}} U^{\prime}\left(x-\xi_{j}\right) U^{\prime}\left(x-\xi_{\ell}\right) d x \\
=\frac{\partial}{\partial \xi_{\ell}}\left[\sum_{j \neq \ell} F\left(\left|\xi_{j}-\xi_{\ell}\right|\right)\right]+O\left(\sigma^{2(1-\beta)-\mu}\right),
\end{gathered}
$$

where $F(r)$ is given by (4.54), and

$$
\begin{aligned}
\left\langle U_{j}^{\prime}, U_{k}^{\prime}\right\rangle \triangleq \int_{\mathbb{R}} U^{\prime}\left(x-\xi_{j}\right) U^{\prime}\left(x-\xi_{\ell}\right) d x \\
=\left\{\begin{array}{l}
6 / 5, \quad \text { for } j=\ell, \\
O\left(\sigma^{|j-\ell|(1-\beta)}|j-\ell||\log \sigma|\right), \quad \text { for } j \neq \ell .
\end{array}\right.
\end{aligned}
$$

Proof. Combining (4.53) in Theorem 4.3 and (5.34) in Lemma 5.3, we get from (5.16) the following equality, for $\ell=1, \ldots, k$,

$$
\begin{aligned}
\sum_{j=1}^{k} \gamma_{j} \int_{\mathbb{R}} U^{\prime}\left(x-\xi_{j}\right) U^{\prime}\left(x-\xi_{\ell}\right) d x & =\int_{\mathbb{R}} S[W] U^{\prime}\left(x-\xi_{\ell}\right) d x \\
+\int_{\mathbb{R}}\left(S\left[W+\varphi_{\sigma, \xi}\right]-S[W]\right) U^{\prime}\left(x-\xi_{\ell}\right) d x & \\
=\frac{\partial}{\partial \xi_{\ell}}\left[\sum_{j \neq \ell} F\left(\left|\xi_{j}-\xi_{\ell}\right|\right)\right]+ & O\left(\sigma^{2(1-\beta)}|\log \sigma|^{2}\right)+O\left(\sigma^{2(1-\beta)-\mu}\right) \\
& =\frac{\partial}{\partial \xi_{\ell}}\left[\sum_{j \neq \ell} F\left(\left|\xi_{j}-\xi_{\ell}\right|\right)\right]+O\left(\sigma^{2(1-\beta)-\mu}\right),
\end{aligned}
$$

where the term $O\left(\sigma^{2(1-\beta)}|\log \sigma|^{2}\right)$ is absorbed by the last term. Moreover, we have

$$
\left\langle U_{j}^{\prime}, U_{j}^{\prime}\right\rangle=\int_{\mathbb{R}}\left|U^{\prime}(x)\right|^{2} d x=6 / 5, \quad j=1, \ldots, k,
$$


by Lemma 1.1. If $j \neq \ell$, then

$$
\begin{aligned}
\left\langle U_{j}^{\prime}, U_{\ell}^{\prime}\right\rangle \leq & 36 \int_{\mathbb{R}} e^{-|x|} e^{-\left|x+\xi_{j}-\xi_{\ell}\right|} d x \quad(\text { by }(1.7)) \\
& =36\left(1+\left|\xi_{j}-\xi_{\ell}\right|\right) e^{-\left|\xi_{j}-\xi_{\ell}\right|} \\
\leq & 36(1+|j-\ell||\log \sigma|) \sigma^{|j-\ell|(1-\beta)} \\
= & O\left(\sigma^{|j-\ell|(1-\beta)}|j-\ell||\log \sigma|\right) .
\end{aligned}
$$

The proof is completed.

\section{Solution to the Reduced Finite-Dimensional Problem}

In this section, we shall take the final step of the Lyapunov-Schmidt method to solve the reduced finite-dimensional problem: To find a set of points $\xi=$ $\left(\xi_{1}, \ldots, \xi_{k}\right) \in \Lambda$ such that the unique solution $\gamma_{\sigma, \xi}=\left(\gamma_{1}, \ldots, \gamma_{k}\right)=0 \in \mathbb{R}^{k}$ for (5.16), provided that $\sigma$ is sufficiently small. If we achieve this, then (5.16) implies that there exists a $\varphi_{\sigma, \xi} \in L_{\mu}^{\infty}$, which satisfies

$$
\begin{gathered}
S\left[W+\varphi_{\sigma, \xi}\right](x)=0, \quad x \in \mathbb{R}, \\
\left\langle\varphi_{\sigma, \xi}, U^{\prime}\left(\cdot-\xi_{j}\right)\right\rangle=0, \quad j=1, \ldots, k, \\
\varphi_{\sigma, \xi}(x) \rightarrow 0, \text { as }|x| \rightarrow \infty .
\end{gathered}
$$

This means $u=W+\varphi_{\sigma, \xi}$ is a solution of the original nonlocal problem (2.5), i.e.,

$$
\begin{gathered}
S[u]=u^{\prime \prime}-u+\frac{u^{2}}{T\left[u^{2}\right]}=0 \text { in } \mathbb{R}, \\
u>0 \text { and } u \rightarrow 0 \text { as }|x| \rightarrow \infty .
\end{gathered}
$$

The key term in (5.35) is

$$
\begin{gathered}
\frac{\partial}{\partial \xi_{\ell}}\left[\sum_{j \neq \ell} F\left(\left|\xi_{j}-\xi_{\ell}\right|\right)\right]=-\frac{\partial}{\partial \xi_{\ell}}\left[\sum_{j \neq \ell} d(\sigma) \sigma^{1+\beta}\left|\xi_{j}-\xi_{\ell}\right|\right] \\
-\frac{\partial}{\partial \xi_{\ell}}\left[\sum_{j \neq \ell} 36 e^{-\left|\xi_{j}-\xi_{\ell}\right|}\right] \\
=\sum_{j \neq \ell} d(\sigma) \sigma^{1+\beta} \operatorname{sgn}\left(\xi_{j}-\xi_{\ell}\right)+\sum_{j \neq \ell} 36 e^{-\left|\xi_{j}-\xi_{\ell}\right|} \operatorname{sgn}\left(\xi_{\ell}-\xi_{j}\right) \\
=d(\sigma) \sigma^{1+\beta}(k+1-2 \ell)-\sum_{j \neq \ell} 36 e^{\left|\xi_{j}-\xi_{\ell}\right|} \operatorname{sgn}\left(\xi_{j}-\xi_{\ell}\right), \ell=1, \ldots, k .
\end{gathered}
$$

Choose $k$ points $\xi_{1}^{0}, \ldots, \xi_{k}^{0}$ such that the following conditions are satisfied

$$
\sum_{j=1}^{k} \xi_{j}^{0}=0,
$$

and

(6.4b) $\xi_{j}^{0}-\xi_{j+1}^{0}=-\left|\xi_{j}^{0}-\xi_{j+1}^{0}\right|=(1+\beta) \log \sigma+\log [j(k-j)]+\log \frac{d(\sigma)}{36}$,

$$
\text { for } j=1, \ldots, k-1 \text {. }
$$


Denote these points by $\xi^{0}=\left(\xi_{1}^{0}, \ldots, \xi_{k}^{0}\right)$. Since $k=C \sigma^{-\beta}$, we have

$$
\max _{1 \leq j \leq k-1} j(k-j) \leq \frac{k^{2}}{4}=\left(\frac{C}{2}\right)^{2} \sigma^{-2 \beta}
$$

so that

$$
\begin{aligned}
\left|\xi_{j}^{0}-\xi_{j+1}^{0}\right| & =-(1+\beta) \log \sigma-\log [j(k-j)]-\log \frac{d(\sigma)}{36} \\
& \geq(1-\beta)|\log \sigma|-2 \log \frac{C}{2}-\log \frac{d(\sigma)}{36}
\end{aligned}
$$

By (4.12) and (4.41), for $\sigma$ sufficiently small,

$$
-2 \log \frac{C}{2}-\log \frac{d(\sigma)}{36}=\log \frac{144}{C^{2} d(\sigma)}>\log \left(\frac{144}{C^{2}} \cdot \frac{5 \times 6 C}{12 \times 40}\right)=\log \left(\frac{9}{C}\right) .
$$

If $k$ satisfies

$$
k=9 \sigma^{-\beta},
$$

then we have, for $j=1, \ldots, k$,

$$
\begin{aligned}
(1-\beta)|\log \sigma| & <\left|\xi_{j}^{0}-\xi_{j+1}^{0}\right| \leq-(1+\beta) \log \sigma-\log \frac{(k-1) d(\sigma)}{36} \\
& \approx-(1+\beta) \log \sigma-\log \frac{k d(\sigma)}{36} \quad(\text { by }(4.12) \text { and }(4.41)) \\
& \approx|\log \sigma|-\log \frac{12 \times 36}{5 \times 6 \times 36}=|\log \sigma|+\log \frac{5}{2} \\
& <|\log \sigma|+1 .
\end{aligned}
$$

It is seen from (6.9) that $\xi^{0} \in \Lambda(\sigma, \beta)$. Moreover, if $\eta>0$ is a sufficiently small number, then

$$
\Lambda_{\eta}=\left\{\xi=\left(\xi_{1}, \ldots, \xi_{k}\right):\left\|\xi-\xi^{0}\right\|<\eta, \sum_{j=0}^{k} \xi_{j}=0\right\} \subset \Lambda(\sigma, \beta) .
$$

In fact, this choice of $\xi^{0}$ implies that for $\sigma$ sufficiently small and for $\xi \in \Lambda_{\eta}$,

$$
\begin{gathered}
-\sum_{j \neq \ell} 36 e^{-\left|\xi_{j}-\xi_{\ell}\right|} \operatorname{sgn}\left(\xi_{j}-\xi_{\ell}\right)=-\sum_{j \neq \ell} 36 e^{-\left|\xi_{j}-\xi_{\ell}\right|} \operatorname{sgn}\left(\xi_{j}^{0}-\xi_{\ell}^{0}\right) \\
=36\left[e^{-\left|\xi_{\ell-1}-\xi_{\ell}\right|}-e^{\left|\xi_{\ell+1}-\xi_{\ell}\right|}\right]+O\left(\sigma^{2(1-\beta)}\right) \\
=36\left[e^{-\left|\xi_{\ell-1}^{0}-\xi_{\ell}^{0}+\hat{\xi}_{\ell-1}-\hat{\xi}_{\ell}\right|}-e^{-\left|\xi_{\ell+1}^{0}-\xi_{\ell}^{0}+\hat{\xi}_{\ell+1}-\hat{\xi}_{\ell}\right|}\right]+O\left(\sigma^{2(1-\beta)}\right),
\end{gathered}
$$

where

$$
\xi_{j}=\xi_{j}^{0}+\hat{\xi}_{j}, \quad j=1, \ldots, k, \text { and } \sum_{j=0}^{k} \hat{\xi}_{j}=0 .
$$

By Taylor expansions, we get

$$
\begin{aligned}
e^{-\left|\xi_{j}^{0}-\xi_{\ell}^{0}+\hat{\xi}_{j}-\hat{\xi}_{\ell}\right|}= & e^{-\left|\xi_{j}^{0}-\xi_{\ell}^{0}\right|}+e^{-\left|\xi_{j}^{0}-\xi_{\ell}^{0}\right|}\left(\operatorname{sgn}\left(\xi_{\ell}-\xi_{j}\right)\right)\left(\hat{\xi}_{j}-\hat{\xi}_{\ell}\right) \\
& +O\left(\sigma\left|\hat{\xi}_{j}-\hat{\xi}_{\ell}\right|^{2}\right)
\end{aligned}
$$


for $|j-\ell|=1$. Substituting (6.12) into (6.11), we obtain

$$
\begin{gathered}
-\sum_{j \neq \ell} 36 e^{-\left|\xi_{j}-\xi_{\ell}\right|} \operatorname{sgn}\left(\xi_{j}-\xi_{\ell}\right)=36\left[e^{-\left|\xi_{\ell-1}^{0}-\xi_{\ell}^{0}\right|}-e^{-\left|\xi_{\ell+1}^{0}-\xi_{\ell}^{0}\right|}\right] \\
+36\left[e^{-\left|\xi_{\ell-1}^{0}-\xi_{\ell}\right|}\left(\hat{\xi}_{\ell-1}-\hat{\xi}_{\ell}\right)+e^{-\left|\xi_{\ell+1}^{0}-\xi_{\ell}^{0}\right|}\left(\hat{\xi}_{\ell+1}-\hat{\xi}_{\ell}\right)\right] \\
+O\left(\sigma\left[\left|\hat{\xi}_{\ell-1}-\hat{\xi}_{\ell}\right|^{2}+\left|\hat{\xi}_{\ell+1}-\hat{\xi}_{\ell}\right|^{2}\right]\right)+O\left(\sigma^{2(1-\beta)}\right),
\end{gathered}
$$

where

$$
\begin{gathered}
36\left[e^{-\left|\xi_{\ell-1}^{0}-\xi_{\ell}^{0}\right|}-e^{\left.-\left|\xi_{\ell+1}^{0}-\xi_{\ell}^{0}\right|\right]}=d(\sigma) \sigma^{1+\beta}[(\ell-1)(k-\ell+1)-\ell(k-\ell)]\right. \\
=-d(\sigma) \sigma^{1+\beta}(k+1-2 \ell), \quad \ell=1, \ldots, k .
\end{gathered}
$$

From (6.3), (6.14) and (6.15), it is seen that (5.35) becomes

$$
\begin{aligned}
& \sum_{j=1}^{k} \gamma_{j}\left\langle U_{j}^{\prime}, U_{\ell}^{\prime}\right\rangle=\frac{\partial}{\partial \xi_{\ell}}\left[\sum_{j \neq \ell} F\left(\left|\xi_{j}-\xi_{\ell}\right|\right)\right]+O\left(\sigma^{2(1-\beta)-\mu}\right) \\
& =36\left[e^{-\left|\xi_{\ell-1}^{0}-\xi_{\ell}^{0}\right|}\left(\hat{\xi}_{\ell-1}-\hat{\xi}_{\ell}\right)+e^{-\left|\xi_{\ell+1}^{0}-\xi_{\ell}^{0}\right|}\left(\hat{\xi}_{\ell+1}-\hat{\xi}_{\ell}\right)\right] \\
& +O\left(\sigma\left[\left|\hat{\xi}_{\ell-1}-\hat{\xi}_{\ell}\right|^{2}+\left|\hat{\xi}_{\ell+1}-\hat{\xi}_{\ell}\right|^{2}\right]\right)+O\left(\sigma^{2(1-\beta)-\mu}\right)
\end{aligned}
$$

where $\left\langle U_{j}^{\prime}, U_{\ell}^{\prime}\right\rangle, j, \ell=1, \ldots, k$, satisfy (5.36). For notational convenience, set

$$
m(i, j)=e^{-\left|\xi_{i}^{0}-\xi_{j}^{0}\right|}, \quad i, j=1, \ldots, k
$$

The system of equations (6.16) for $\gamma=\left(\gamma_{1}, \ldots, \gamma_{k}\right)$ can be written as

$$
\begin{gathered}
\left(\begin{array}{c}
\sum_{j=1}^{k} \gamma_{j}\left\langle U_{j}^{\prime}, U_{1}^{\prime}\right\rangle \\
\vdots \\
\sum_{j=1}^{k} \gamma_{j}\left\langle U_{j}^{\prime}, U_{k}^{\prime}\right\rangle
\end{array}\right)=M\left(\begin{array}{c}
\hat{\xi}_{1} \\
\vdots \\
\hat{\xi}_{k}
\end{array}\right) \\
+O\left(\sigma\left[\left|\hat{\xi}_{\ell-1}-\hat{\xi}_{\ell}\right|+\left|\hat{\xi}_{\ell+1}-\hat{\xi}_{\ell}\right|\right]\right)+O\left(\sigma^{2(1-\beta)-\mu}\right),
\end{gathered}
$$


where $M$ is the $k \times k$ matrix as follows,

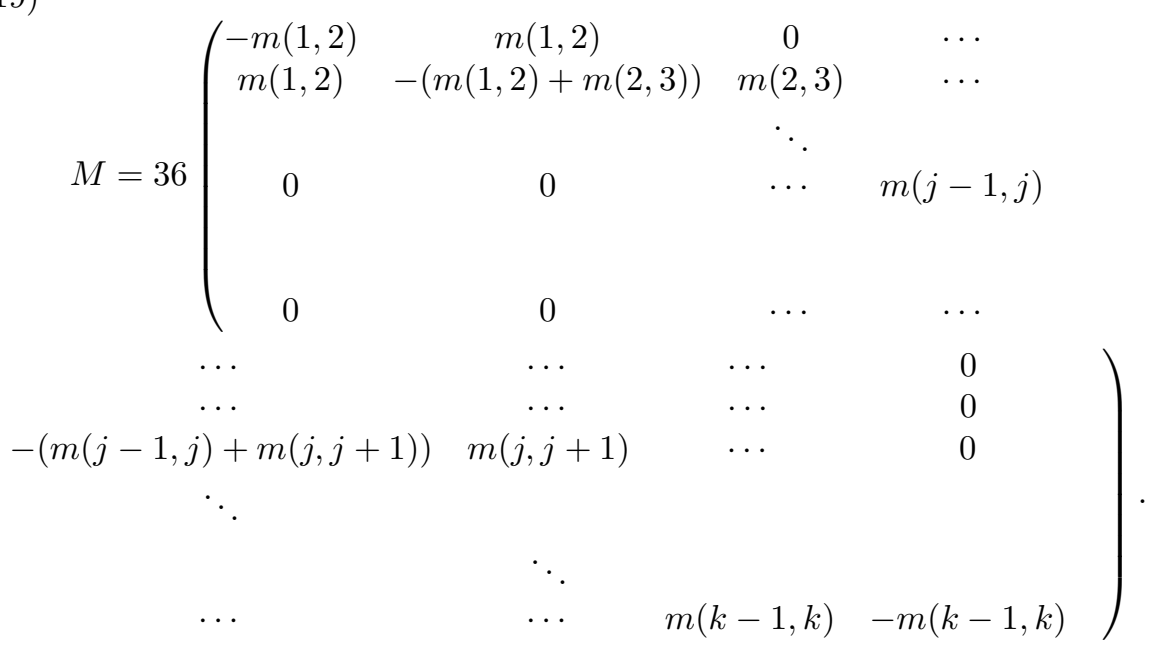

LEMMA 6.1. The symmetric matrix $M$ has an eigenvalue $\lambda_{1}=0$, whose eigenspace $N_{1}$ is one-dimensional,

$$
N_{1}=\left\{\hat{\xi}=(s, s, \ldots, s) \in \mathbb{R}^{k} \mid s \neq 0\right\} .
$$

On the orthogonal complement subspace

$$
N_{1}^{\perp}=\left\{\hat{\xi}=\left(\hat{\xi}_{1}, \ldots, \hat{\xi}_{k}\right) \in \mathbb{R}^{k} \mid \sum_{j=1}^{k} \hat{\xi}_{j}=0\right\},
$$

the matrix $M$ is invertible and the operator norm of the inverse matrix $M^{-1}$ on $N_{1}^{\perp}$ satisfies

$$
\left\|M^{-1}\right\| \leq \tilde{C} \sigma^{-1}
$$

for some uniform constant $\tilde{C}>0$.

Proof. In the matrix $\lambda I-M$, adding all the 2 nd through $k$ th rows to the first row, we get

$$
\operatorname{det}(\lambda I-M)=\lambda \operatorname{det} M_{1}(\lambda)
$$

where $M_{1}(\lambda)$ is another $\lambda$-matrix. This shows $\lambda_{1}=0$ is an eigenvalue of $M$. It can be shown directly that its associated eigenspace $N_{1}$ is characterized by (6.20). For any $\hat{\xi} \in N_{1}^{\perp}$,

$$
\sum_{j=1}^{k} s \hat{\xi}_{j}=0 \quad \text { so that }(6.21) \text { holds, }
$$

and vice-versa. Since $M$ is symmetric, beside $\lambda_{1}=0$, all the other $k-1$ eigenvalues must be real numbers.

Let $\lambda_{\min }$ be a nonzero eigenvalue of $M$ on $N_{1}^{\perp}$, which has the smallest absolute value. Then we know that

$$
\left|\lambda_{\min }\right|=\inf _{\substack{x \in N_{1}^{\perp} \\\|x\|=1}}\{|\langle M x, x\rangle|\} .
$$


For any $x=\operatorname{col}\left(x_{1}, \ldots, x_{k}\right) \in \mathbb{R}^{k}$ with $\|x\|=1$, we have

$$
\begin{gathered}
\frac{1}{36}\langle M x, x\rangle=-m(1,2)\left(x_{1}-x_{2}\right) x_{1}+m(1,2)\left(x_{1}-x_{2}\right) x_{2} \\
-m(2,3)\left(x_{2}-x_{3}\right) x_{2}+m(2,3)\left(x_{2}-x_{3}\right) x_{3} \\
\ldots \ldots \\
-m(j-1, j)\left(x_{j-1}-x_{j}\right) x_{j-1}+m(j-1, j)\left(x_{j-1}-x_{j}\right) x_{j} \\
\ldots \ldots \\
-m(k-1, k)\left(x_{k-1}-x_{k}\right) x_{k-1}+m(k-1, k)\left(x_{k-1}-x_{k}\right) x_{k} \\
=-\sum_{j=1}^{k-1} m(j, j+1)\left(x_{j}-x_{j+1}\right)^{2} \leq 0,
\end{gathered}
$$

here we know that

$$
m(j, j+1)=e^{-\left|\xi_{j}^{0}-\xi_{j+1}^{0}\right|}>0 .
$$

Hence, by (6.4b) and (6.23), we have

$$
\begin{aligned}
\left|\lambda_{\min }\right| & =-\lambda_{\min }=\inf \left\{36 \sum_{j=1}^{k-1} m(j, j+1)\left(x_{j}-x_{j+1}\right)^{2}: x \in N_{1}^{\perp},\|x\|=1\right\} \\
& =36 m(1,2)=36 m(k-1, k)=d(\sigma) \sigma^{1+\beta}\left(9 \sigma^{-\beta}-1\right) \geq C \sigma
\end{aligned}
$$

for some constant $C>0$. Thus there is a constant $\tilde{C}>0$, such that on the subspace $N_{1}^{\perp}$, the norm of the inverse operator $M^{-1}$ satisfies

$$
\left\|M^{-1}\right\|=\frac{1}{\left|\lambda_{\min }\right|} \leq \tilde{C} \sigma^{-1}
$$

Note that for $k=2$, the matrix $M$ has the eigenvalues $\lambda_{1}=0$ and $\lambda_{2}=$ $-12 m(1,2)$. But this pattern is not true for $k \geq 3$ in general. For $k=3$, the three eigenvalues are $\lambda_{1}=0$ and

$$
\lambda_{2}, \lambda_{3}=m(1,2)+m(2,3) \pm \sqrt{m(1,2)^{2}-m(1,2) m(2,3)+m(2,3)^{2}} .
$$

However, (6.22) always holds.

THEOREM 6.2. Under the assumption that

$$
k=9 \sigma^{-\beta} \text {, with } \beta<\frac{1}{2} \text { and arbitrarily close to } \frac{1}{2},
$$

there is a constant $\sigma^{*}>0$, such that for any given $0<\sigma \leq \sigma^{*}$, there exists a $\xi=\left(\xi_{1}, \ldots, \xi_{k}\right) \in \Lambda=\Lambda(\sigma, \beta)$ such that the problem (5.16) has a unique solution $\left(\varphi_{\sigma, \xi}, \gamma_{\sigma, \xi}\right)$, where

$$
\gamma_{\sigma, \xi}=\left(\gamma_{1}, \ldots, \gamma_{k}\right)=0
$$

ProOF. According to Theorem 5.4, the unique solution $\left(\varphi_{\sigma, \xi}, \gamma_{\sigma, \xi}\right)$ to the problem (5.16) satisfies Eq. (5.35), which is equivalently reduced to the system (6.18). By (5.36), we can show that the coefficient matrix on the left-hand side of (6.18),

$$
\left(\begin{array}{ccc}
\left\langle U_{1}^{\prime}, U_{1}^{\prime}\right\rangle & \cdots & \left\langle U_{k}^{\prime}, U_{1}^{\prime}\right\rangle \\
\cdots & & \cdots \\
\left\langle U_{1}^{\prime}, U_{k}^{\prime}\right\rangle & \cdots & \left\langle U_{k}^{\prime}, U_{k}^{\prime}\right\rangle
\end{array}\right)=\frac{6}{5} I+A
$$


is the same coefficient matrix in (3.16) and it is nonsingular. We can solve (6.18) for $\gamma_{\sigma, \xi}$ and get

$$
\gamma_{\sigma, \xi}=\left(\frac{6}{5} I+A\right)^{-1} M\left\{\left(\begin{array}{c}
\hat{\xi}_{1} \\
\vdots \\
\hat{\xi}_{k}
\end{array}\right)-\Psi_{\sigma}(\hat{\xi})\right\},
$$

where the mapping $\Psi_{\sigma}$ is defined by

$$
\Psi_{\sigma}(\hat{\xi})=-M^{-1}\left\{O\left(\sigma\left[\left|\hat{\xi}_{\ell-1}-\hat{\xi}_{\ell}\right|^{2}+\left|\hat{\xi}_{\ell+1}-\hat{\xi}_{\ell}\right|^{2}\right]\right)+O\left(\sigma^{2(1-\beta)-\mu}\right)\right\},
$$

in which, of course, the two terms inside $\{\cdots\}$ are $k$-dimensional column vectors whose $\ell$ th components are as shown, respectively.

In order to show (6.26), it suffices to prove that there is a small number $\eta>0$ such that

$$
\Psi_{\sigma} \text { has a fixed point } \hat{\xi} \in \Lambda_{\eta}\left(\subset N_{1}^{\perp}\right) .
$$

Now we use the argument of contraction mapping to prove it. Take a set

$$
Z=\left\{\hat{\xi} \in N_{1}^{\perp}:\|\hat{\xi}\| \leq \eta=|\log \sigma|^{-\nu}\right\}
$$

where $\nu>0$ is a small positive number and let $\nu$ be fixed. For any fixed $\beta$ such that

$$
0<\beta<\frac{1}{2}, \text { arbitrarily close to } \frac{1}{2},
$$

a value of the parameter $\mu$ can be chosen such that

$$
0<\mu<\min \left\{\frac{1}{3}, 1-2 \beta\right\}
$$

which implies that

$$
\mu<\frac{1-2 \beta}{1-\beta} \text { so that (3.63) is satisfied: } \beta<\frac{1-\mu}{2-\mu} .
$$

Let $\mu$ be fixed in such a way. Then we have, by Lemma 6.1 and (6.22),

$$
\begin{gathered}
\left\|\Psi_{\sigma}(\hat{\xi})\right\|_{\mathbb{R}^{k}} \leq\left\|M^{-1}\right\|\left\{\left\|O\left(\sigma\left[\left|\hat{\xi}_{\ell-1}-\hat{\xi}_{\ell}\right|^{2}+\left|\hat{\xi}_{\ell+1}-\hat{\xi}_{\ell}\right|^{2}\right]\right)\right\|\right. \\
\left.+\left\|O\left(\sigma^{2(1-\beta)-\mu}\right)\right\|\right\} \leq C^{*}\left\{\|\hat{\xi}\|^{2}+\sigma^{1-2 \beta-\mu}\right\} \\
\leq C^{*}\left\{|\log \sigma|^{-\nu}+\sigma^{1-2 \beta-\mu}|\log \sigma|^{\nu}\right\}|\log \sigma|^{-\nu} .
\end{gathered}
$$

Then there exists a constant $\sigma_{1}>0$ such that, whenever $0<\sigma \leq \sigma_{1}$, in (6.32) we have

$$
C^{*}\left\{|\log \sigma|^{-\nu}+\sigma^{1-2 \beta-\mu}|\log \sigma|^{\nu}\right\}<1 .
$$

Therefore, for any $0<\sigma \leq \sigma_{1}$, we have

$$
\Psi_{\sigma}(Z) \subset Z \text {. }
$$


Next, for any $\hat{\xi}$ and $\hat{\zeta}$ in $Z$, we have

$$
\begin{aligned}
& \left\|\Psi_{\sigma}(\hat{\xi})-\Psi_{\sigma}(\hat{\zeta})\right\|_{\mathbb{R}^{k}} \leq C^{*} \| \operatorname{col}\left(\left|\hat{\xi}_{\ell-1}-\hat{\xi}_{\ell}\right|^{2}-\left|\hat{\zeta}_{\ell-1}-\hat{\zeta}_{\ell}\right|^{2} \mid\right. \\
& \left.+|| \hat{\xi}_{\ell+1}-\left.\hat{\xi}_{\ell}\right|^{2}-\left|\hat{\zeta}_{\ell+1}-\hat{\zeta}_{\ell}\right|^{2} \mid\right) \| \\
& \leq 2 C^{*}(\|\hat{\xi}\|+\|\hat{\zeta}\|) \| \operatorname{col}\left(\left\|\hat{\xi}_{\ell-1}-\hat{\xi}_{\ell}|-| \hat{\zeta}_{\ell-1}-\hat{\zeta}_{\ell}\right\|\right. \\
& \left.+\left\|\hat{\xi}_{\ell+1}-\hat{\xi}_{\ell}|-| \hat{\zeta}_{\ell+1}-\hat{\zeta}_{\ell}\right\|\right) \| \\
& \quad(\text { by the triangle inequalities) } \\
& \leq 2 C^{*}(\|\hat{\xi}\|+\|\hat{\zeta}\|)\left\|\operatorname{col}\left(\left|\hat{\xi}_{\ell-1}-\hat{\zeta}_{\ell-1}\right|+2\left|\hat{\xi}_{\ell}-\hat{\zeta}_{\ell}\right|+\left|\hat{\xi}_{\ell+1}-\hat{\zeta}_{\ell+1}\right|\right)\right\| \\
& \leq 8 C^{*}(\|\hat{\xi}\|+\|\hat{\zeta}\|)\|\hat{\xi}-\hat{\zeta}\| \\
& \leq 16 C^{*}|\log \sigma|^{-\nu}\|\hat{\xi}-\hat{\zeta}\| .
\end{aligned}
$$

There exists a small constant $\sigma_{2}>0$ such that, whenever $0<\sigma \leq \sigma_{2}$, in (6.35) we have

$$
16 C^{*}|\log \sigma|^{-\nu} \leq \frac{1}{2}
$$

so that

$$
\left\|\Psi_{\sigma}(\hat{\xi})-\Psi_{\sigma}(\hat{\zeta})\right\| \leq \frac{1}{2}\|\hat{\xi}-\hat{\zeta}\| \text {, for any } \hat{\xi}, \hat{\zeta} \in Z \text {. }
$$

Therefore, by (6.34) and (6.37), $\Psi_{\sigma}$ is a contraction mapping on the set $Z$, provided that

$$
0<\sigma<\min \left\{\sigma_{1}, \sigma_{2}\right\} .
$$

By Brouwer's fixed point theorem, there exists a fixed point

$$
\hat{\xi}_{\sigma} \in Z \text { such that } \hat{\xi}_{\sigma}=\Psi_{\sigma}\left(\hat{\xi}_{\sigma}\right),
$$

and consequently, this $\hat{\xi}_{\sigma}$ yields

$$
\gamma_{\sigma, \xi_{\sigma}}=\left(\gamma_{1}, \ldots, \gamma_{k}\right)=0,
$$

in (6.27), with $\xi_{\sigma}=\xi^{0}+\hat{\xi}_{\sigma}$. Thus, Theorem 6.2 is proved with

$$
\sigma^{*}=\min \left\{\sigma_{1}, \sigma_{2}\right\} \text {. }
$$

The proof is completed.

Conclusion (The completion of the proof of Theorem 2.1). Let

$$
\sigma_{0}=\min \left\{\hat{\sigma}, \check{\sigma}, \sigma^{*}\right\},
$$

where $\hat{\sigma} \leq \frac{1}{2}, \check{\sigma}$, and $\sigma^{*}$ are determined in Theorem 3.6, Theorem 5.2, and Theorem 6.2 , respectively. For any $0<\sigma \leq \sigma_{0}$, all the results shown in Sections 3 through 6 are valid. Therefore, there is a multi-spike solution $(u, v)$ to the Gierer-Meinhardt system (2.3), in which

$$
u=W+\varphi_{\sigma, \xi_{\sigma}} \quad \text { and } V=T\left[u^{2}\right],
$$


where $W(x)$ is given by (1.17), $\varphi_{\sigma, \xi_{\sigma}} \in L_{\mu}^{\infty}$ is the solution of problem (5.16) together with $\gamma_{\sigma, \xi_{\sigma}}=0$. Hence the property (2.8) is valid. The property (2.9) for the $v$ component is valid because

$$
\begin{gathered}
v=T\left[u^{2}\right]=T\left[W^{2}\right]+2 T\left[W \varphi_{\sigma, \xi_{\sigma}}\right]+T\left[\varphi_{\sigma, \xi_{\sigma}}^{2}\right] \\
=V(x)+2 T\left[W \varphi_{\sigma, \xi_{\sigma}}\right]+T\left[\varphi_{\sigma, \xi_{\sigma}}^{2}\right]
\end{gathered}
$$

and by Lemmas 4.1 and 4.2, (5.6) and (5.7), with a little extension, we have

$$
\begin{gathered}
\left|v\left(\xi_{j}+x\right)-1\right| \leq\left|V\left(\xi_{j}+x\right)-V\left(\xi_{j}\right)\right| \\
+\left|V\left(\xi_{j}\right)-1\right|+2\left|T\left[W \varphi_{\sigma, \xi_{\sigma}}\right]\right|+\left|T\left[\varphi_{\sigma, \xi_{\sigma}}^{2}\right]\right| \\
=\left|V\left(\xi_{j}+x\right)-V\left(\xi_{j}\right)\right|+\left|V\left(\xi_{j}\right)-1\right|+O\left(\sigma^{\beta}\right)\left\|\varphi_{\sigma, \xi_{\sigma}}\right\|_{\mu} \\
+O(1)\left\|\varphi_{\sigma, \xi_{\sigma}}\right\|_{\mu}^{2} \rightarrow 0
\end{gathered}
$$

as $\sigma \rightarrow 0$, uniformly on any compact set of $x$. Thus Theorem 2.1 is proved. 


\section{Appendix A}

We can solve the nonlinear ODE problem:

$$
\begin{gathered}
u^{\prime \prime}-u+u^{2}=0 \quad \text { in } \mathbb{R}, \\
0<u(x) \rightarrow 0, \text { as }|x| \rightarrow \infty,
\end{gathered}
$$

to get the unique explicit solution

$$
u(x)=6 \operatorname{sech}^{2}\left(\frac{x}{2}\right)=\frac{6 e^{x}}{\left(1+e^{x}\right)^{2}} .
$$

By multiplying $u^{\prime}$ on both sides of the equation and integrating it, we have

$$
\left(u^{\prime}\right)^{2}=u^{2}-\frac{2}{3} u^{3}
$$

so that

$$
u^{\prime}=-u \sqrt{1-\frac{2}{3} u}
$$

The sign is chosen according to the condition $0<u(x) \rightarrow 0$, as $|x| \rightarrow \infty$. Let $y=\log u$. Then

$$
\int \frac{d y}{\sqrt{1-\frac{2}{3} e^{y}}}=-x
$$

where we do not involve an arbitrary constant also because of the asymptotical boundary condition. Another substitution $z=\sqrt{1-\frac{2}{3} e^{y}}, d z=\frac{-1 / 3 e^{y} d y}{\sqrt{1-\frac{2}{3} e^{y}}}$, renders the above equality to

$$
\int \frac{-2 d z}{1-z^{2}}=(-1) \int\left(\frac{1}{1-z}+\frac{1}{1+z}\right) d z=-x,
$$

or

$$
x=\log \frac{1+z}{1-z}, \quad \text { i.e., } \quad z=\frac{e^{x}-1}{e^{x}+1}=\sqrt{1-\frac{2}{3} u} .
$$

Then solve this algebraic equation for $u$, finally we obtain the unique solution

$$
u(x)=\frac{6 e^{x}}{\left(1+e^{x}\right)^{2}}, \quad x \in \mathbb{R} .
$$

\section{Appendix B}

In this Appendix we compute the integrals in Lemma 1.1. First,

$$
\begin{gathered}
\int_{\mathbb{R}} U^{2}(x) d x=\int_{\mathbb{R}} \frac{36 e^{-2|x|}}{\left(1+e^{-|x|}\right)^{4}} d x=72 \int_{-\infty}^{0} \frac{e^{2 x}}{\left(1+e^{x}\right)^{4}} d x \quad\left(\text { let } y=1+e^{x}\right) \\
=72 \int_{1}^{2} \frac{y-1}{y^{4}} d y=\left.72\left\{\frac{-1}{2 y^{2}}+\frac{1}{3 y^{3}}\right\}\right|_{1} ^{2}=72\left[\frac{1}{2}\left(1-\frac{1}{4}\right)+\frac{1}{3}\left(\frac{1}{8}-1\right)\right] \\
=6 .
\end{gathered}
$$


Next,

$$
\begin{aligned}
\int_{\mathbb{R}} U^{3}(x) d x & =\int_{\mathbb{R}} \frac{6^{3} e^{-3|x|}}{\left(1+e^{-|x|}\right)^{6}} d x=432 \int_{-\infty}^{0} \frac{e^{3 x}}{\left(1+e^{x}\right)^{6}} d x \quad\left(\text { let } y=1+e^{x}\right) \\
& =432 \int_{1}^{2} \frac{(y-1)^{2}}{y^{6}} d y=432 \int_{1}^{2}\left[\frac{1}{y^{4}}-\frac{2}{y^{5}}+\frac{1}{y^{6}}\right] d y \\
=432 & {\left.\left[-\frac{1}{3 y^{3}}+\frac{1}{2 y^{4}}-\frac{1}{5 y^{5}}\right]\right|_{1} ^{2}=432\left(\frac{7}{24}-\frac{15}{32}+\frac{31}{160}\right)=\frac{36}{5} }
\end{aligned}
$$

Now we compute $\int_{\mathbb{R}}\left|U^{\prime}(x)\right|^{2} d x$.

$$
\begin{gathered}
\int_{\mathbb{R}}\left|U^{\prime}(x)\right|^{2} d x=72 \int_{-\infty}^{0} \frac{e^{2 x}\left(1-e^{x}\right)^{2}}{\left(1+e^{x}\right)^{6}} d x \quad\left(\text { by substitution } y=1+e^{x}\right) \\
=72 \int_{1}^{2} \frac{(y-1)(y-2)^{2}}{y^{6}} d y=72 \int_{1}^{2} \frac{1}{y^{6}}\left(y^{3}-5 y^{2}+8 y-4\right) d y \\
=72 \int_{1}^{2}\left(y^{-3}-5 y^{-4}+8 y^{-5}-4 y^{-6}\right) d y \\
=\left.72\left[-\frac{1}{2} y^{-2}+\frac{5}{3} y^{-3}-2 y^{-4}+\frac{4}{5} y^{-5}\right]\right|_{1} ^{2} \\
=72\left(\frac{3}{8}-\frac{35}{24}+\frac{15}{8}-\frac{31}{40}\right)=\frac{6}{5} .
\end{gathered}
$$

Then we have

$$
\begin{gathered}
\int_{\mathbb{R}}|x| U(x) d x=2 \int_{0}^{\infty} x U(x) d x=12 \int_{0}^{\infty} \frac{x e^{x}}{\left(1+e^{x}\right)^{2}} d x \\
=12\left[\left.\frac{-x}{1+e^{x}}\right|_{0} ^{\infty}+\int_{0}^{\infty} \frac{d x}{1+e^{x}}\right]=12 \int_{0}^{\infty} \frac{e^{-x}}{1+e^{-x}} d x \\
=-\left.12 \log \left(1+e^{-x}\right)\right|_{0} ^{\infty}=12 \log 2 .
\end{gathered}
$$

Next,

$$
\begin{gathered}
\int_{\mathbb{R}}|x| U^{2}(x) d x=72 \int_{0}^{\infty} \frac{x e^{-2 x}}{\left(1+e^{-x}\right)^{4}} d x=72 \int_{0}^{\infty} \frac{x e^{2 x}}{\left(1+e^{x}\right)^{4}} d x \\
=72\left\{-\left.\frac{1}{3} \frac{x e^{x}}{\left(1+e^{x}\right)^{3}}\right|_{0} ^{\infty}+\frac{1}{3} \int_{0}^{\infty} \frac{(1+x) e^{x}}{\left(1+e^{x}\right)^{3}} d x\right\} \\
=72\left\{-\left.\frac{1}{6} \frac{1+x}{\left(1+e^{x}\right)^{2}}\right|_{0} ^{\infty}+\frac{1}{6} \int_{0}^{\infty} \frac{d x}{\left(1+e^{x}\right)^{2}}\right\} \\
=12\left\{\frac{1}{4}+\int_{0}^{\infty} \frac{e^{-2 x}}{\left(1+e^{-x}\right)^{2}} d x\right\}=3+12\left\{\left.\frac{e^{-x}}{1+e^{-x}}\right|_{0} ^{\infty}+\int_{0}^{\infty} \frac{e^{-x}}{1+e^{-x}} d x\right\} \\
=3+12\left\{-\frac{1}{2}-\left.\log \left(1+e^{-x}\right)\right|_{0} ^{\infty}\right\} \\
=3-6+12 \log 2=12 \log 2-3 .
\end{gathered}
$$


Moreover, we have

$$
\begin{gathered}
\int_{\mathbb{R}} e^{-|x|} U^{2}(x) d x=2 \int_{0}^{\infty} e^{-|x|} U^{2}(x) d x=72 \int_{0}^{\infty} \frac{e^{-x} e^{2 x}}{\left(1+e^{x}\right)^{4}} d x \\
=72 \int_{0}^{\infty} \frac{e^{x}}{\left(1+e^{x}\right)^{4}} d x=-\left.\frac{72}{3} \frac{1}{\left(1+e^{x}\right)^{3}}\right|_{0} ^{\infty}=3
\end{gathered}
$$

Finally,

$$
\int_{\mathbb{R}} e^{-x} U^{2}(x) d x=\int_{-\infty}^{\infty} 36 \frac{e^{-x} e^{2 x}}{\left(1+e^{x}\right)^{4}} d x=-\left.\frac{36}{3} \frac{1}{\left(1+e^{x}\right)^{3}}\right|_{-\infty} ^{\infty}=12 .
$$

\section{Appendix C}

Here we give the detailed proof of Lemma 1.2. The first statement in (1.7),

$$
p_{r}(x) \leq e^{-|r|}, \quad x \in \mathbb{R},
$$

simply follows from the triangle inequality. Now we show the second statement in (1.7) by calculation for both cases $r \geq 0$ and $r<0$. For $r \geq 0$, by the explicit expression of $p_{r}(x)$,

$$
\begin{gathered}
\int_{\mathbb{R}} e^{-|x|} e^{-|x+r|} d x=\int_{-\infty}^{-r} e^{2 x+r} d x+\int_{-r}^{0} e^{-r} d x+\int_{0}^{\infty} e^{-(2 x+r)} d x \\
=\frac{1}{2} e^{-r}+r e^{-r}+\frac{1}{2} e^{-r}=(1+|r|) e^{-|r|}
\end{gathered}
$$

For $r<0$,

$$
\begin{gathered}
\int_{\mathbb{R}} e^{-|x|} e^{-|x+r|} d x=\int_{-\infty}^{0} e^{2 x+r} d x+\int_{0}^{-r} e^{r} d x+\int_{-r}^{\infty} e^{-(2 x+r)} d x \\
=\frac{1}{2} e^{r}-r e^{r}+\frac{1}{2} e^{r}=(1+|r|) e^{-|r|}
\end{gathered}
$$

Therefore, (1.7) holds.

To save space, we show (1.8), (1.9), and (1.10) only for the case $r \geq 0$. For the case $r<0$, the verification is similar and is omitted. Since for $r \geq 0$,

$$
\begin{gathered}
\int_{\mathbb{R}}|x| e^{-|x|} e^{-|x+r|} d x=\int_{-\infty}^{-r}-x e^{2 x+r}+\int_{-r}^{0}-x e^{-r}+\int_{0}^{\infty} x e^{-(2 x+r)} \\
=\left.\left(-\frac{x}{2} e^{2 x+r}+\frac{1}{4} e^{2 x+r}\right)\right|_{-\infty} ^{-r}-\left.\frac{x^{2}}{2} e^{-r}\right|_{-r} ^{0}-\left.\left(\frac{x}{2} e^{-(2 x+r)}+\frac{1}{4} e^{-(2 x+r)}\right)\right|_{0} ^{\infty} \\
=e^{-r}\left(\frac{r}{2}+\frac{1}{4}\right)+\frac{r^{2}}{2} e^{-r}+\frac{1}{4} e^{-r}=\frac{1}{2} e^{-|r|}\left(1+|r|+r^{2}\right)
\end{gathered}
$$

so (1.8) is valid. 
Next we show (1.9). For $r \geq 0$, we have

$$
\begin{gathered}
\int_{\mathbb{R}}|x|^{2} e^{-2|x|} e^{-2|x+r|} d x=\int_{-\infty}^{-r} x^{2} e^{2 x} e^{2 x+2 r}+\int_{-r}^{0} x^{2} e^{-2 r} d x \\
+\int_{0}^{\infty} x^{2} e^{-2 x} e^{-2 x-2 r} d x \\
=\left.e^{2 r}\left(\frac{x^{2}}{4} e^{4 x}-\frac{x}{8} e^{4 x}+\frac{1}{32} e^{4 x}\right)\right|_{-\infty} ^{-r}+\left.\frac{x^{3}}{3} e^{-2 r}\right|_{-r} ^{0} \\
+\left.e^{-2 r}\left(-\frac{x^{2}}{4} e^{-4 x}-\frac{x}{8} e^{-4 x}-\frac{1}{32} e^{-4 x}\right)\right|_{0} ^{\infty} \\
=e^{-2 r}\left\{\left(\frac{r^{2}}{4}+\frac{|r|}{8}+\frac{1}{32}\right)+\frac{|r|^{3}}{3}+\frac{1}{32}\right\} \\
=e^{-2|r|}\left(\frac{1}{16}+\frac{|r|}{8}+\frac{r^{2}}{4}+\frac{|r|^{3}}{3}\right) .
\end{gathered}
$$

Therefore, (1.9) is valid.

Finally we show (1.10). For $r \geq 0$, we have

$$
\begin{gathered}
\left.\int_{\mathbb{R}}|x| e^{-|x|} e^{-2|x+r|} d x \leq e^{-|r|} \int_{\mathbb{R}}|x| e^{-|x+r|} d x \quad \text { (by }(1.7)\right) \\
=e^{-|r|}\left\{\int_{-\infty}^{-r}-x e^{x+r}+\int_{-r}^{0}-x e^{-(x+r)}+\int_{0}^{\infty} x e^{-(x+r)}\right\} \\
=e^{-|r|}\left\{\left.\left(-x e^{x+r}+e^{x+r}\right)\right|_{-\infty} ^{-r}+\left.\left(x e^{-(x+r)}+e^{-(x+r)}\right)\right|_{-r} ^{0}\right. \\
+\left.\left(-x e^{-(x+r)}-e^{-(x+r)}\right)\right|_{0} ^{\infty} \\
=e^{-|r|}\left(r+1+r+e^{-r}-1+e^{-r}\right) \\
=e^{-|r|}\left(2 r+2 e^{-r}\right)=2 e^{-|r|}\left(|r|+e^{-|r|}\right) .
\end{gathered}
$$

Therefore, (1.10) is valid. The proof is completed.

\section{Acknowledgements}

The author thanks Professor Juncheng Wei of CUHK for his invitation in the Fall of 2004 and for his suggestions and stimulating discussions. The author also thanks the Department of Mathematics of CUHK for their kind hospitality.

\section{References}

1. X. Chen and M. Kowalczyk, Dynamics of an interior spike in the Gierer-Meinhardt system, SIAM J. Math. Anal. 33 (2001), no. 1, 172-193 (electronic).

2. E. J. Crampin and P. K. Maini, Reaction-diffusion models for biological pattern formation, Methods Appl. Anal. 8 (2001), no. 3, 415-428.

3. M. del Pino, M. Kowalczyk, and X. Chen, The Gierer \& Meinhardt system: the breaking of homoclinics and multi-bump ground states, Commun. Contemp. Math. 3 (2001), no. 3, 419-439.

4. M. del Pino, M. Kowalczyk, and J. Wei, Multi-bump ground states of the Gierer-Meinhardt system in $\mathbb{R}^{2}$, Ann. Inst. H. Poincaré Anal. Non Linéaire 20 (2003), no. 1, 53-85. 
5. A. Doelman, T. J. Kaper, and H. van der Ploeg, Spatially periodic and aperiodic multi-pulse patterns in the one-dimensional Gierer-Meinhardt equation, Methods Appl. Anal. 8 (2001), no. 3, 387-414.

6. A. Doelman, T. J. Kaper, and P. A. Zegeling, Pattern formation in the one-dimensional Gray-Scott model, Nonlinearity 10 (1997), no. 2, 523-563.

7. A. Gierer and H. Meinhardt, A theory of biological pattern formation, Kybernetik 12 (1972), 30-39.

8. P. Gray and S. K. Scott, Autocatalytic reactions in the isothermal, continuous stirred tank reactor: isolas and other forms of multistability, Chem. Eng. Sci. 38 (1983), 29-43.

9. C. Gui and J. Wei, Multiple interior peak solutions for some singularly perturbed Neumann problems, J. Differential Equations 158 (1999), no. 1, 1-27.

10. Neumann problems, Canad. J. Math. 52 (2000), no. 3, 522-538.

11. D. Iron, M. Ward, and J. Wei, The stability of spike solutions to the one-dimensional GiererMeinhardt model, Phys. D 150 (2001), no. 1-2, 25-62.

12. J. P. Keener, Activators and inhibitors in pattern formation, Stud. Appl. Math. 59 (1978), no. 1, 1-23.

13. C. S. Lin, W.-M. Ni, and I. Takagi, Large amplitude stationary solutions to a chemotaxis system, J. Differential Equations 72 (1988), no. 1, 1-27.

14. H. Meinhardt, Models of biological pattern formation, Acad. Press, London, 1982.

15. C. B. Muratov and V. V. Osipov, Static spike autosolitons in the Gray-Scott model, J. Phys. A 33 (2000), no. 48, 8893-8916.

16. W.-M. Ni, Diffusion, cross-diffusion, and their spike-layer steady states, Notices Amer. Math. Soc. 45 (1998), no. 1, 9-18.

17. W.-M. Ni and I. Takagi, On the Neumann problem for some semilinear elliptic equations and systems of activator-inhibitor type, Trans. Amer. Math. Soc. 297 (1986), no. 1, 351-368.

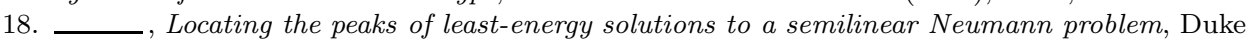
Math. J. 70 (1993), no. 2, 247-281.

19. W.-M. Ni and J. Wei, On the location and profile of spike-layer solutions to singularly perturbed semilinear Dirichlet problems, Comm. Pure Appl. Math. 48 (1995), no. 7, 731-768.

20. I. Takagi, Point-condensation for a reaction-diffusion system, J. Differential Equations 61 (1986), no. 2, 208-249.

21. A. M. Turing, The chemical basis of morphogenesis, Philos. Tran. Royal Soc. London, Ser. B. 237 (1952), 37-72.

22. M. J. Ward and J. Wei, The stability of multiple spikes for the one-dimensional Gray-Scott model, Preprint.

23. __ Asymmetric spike patterns for the one-dimensional Gierer-Meinhardt model: equilibria and stability, European J. Appl. Math. 13 (2002), no. 3, 283-320.

24. The existence and stability of asymmetric spike patterns for the Schnakenberg model, Stud. Appl. Math. 109 (2002), no. 3, 229-264.

25. Hopf bifurcations and oscillatory instabilities of spike solutions for the onedimensional Gierer-Meinhardt model, J. Nonlinear Sci. 13 (2003), no. 2, 209-264.

26. J. Wei, Existence, stability and metastability of point condensation patterns generated by the Gray-Scott system, Nonlinearity 12 (1999), no. 3, 593-616.

27. _ On single interior spike solutions of the Gierer-Meinhardt system: uniqueness and spectrum estimates, European J. Appl. Math. 10 (1999), no. 4, 353-378.

28. _ Point-condensations generated by Gierer-Meinhardt systems: a brief survey, New Trends in Nonlinear Partial Differential Equations (Y. Morita, H. Nonoyama, E. Yanagida, and S. Yotsutani, eds.), 2000, pp. 46-59.

29. _ Pattern formations in two-dimensional Gray-Scott model: existence of single-spot solutions and their stability, Phys. D 148 (2001), no. 1-2, 20-48.

30. J. Wei and M. Winter, On the two-dimensional Gierer-Meinhardt system with strong coupling, SIAM J. Math. Anal. 30 (1999), no. 6, 1241-1263 (electronic).

31. S Sikes for the two-dimensional Gierer-Meinhardt system: the weak coupling case, J. Nonlinear Sci. 11 (2001), no. 6, 415-458.

32. Spikes for the Gierer-Meinhardt system in two dimensions: the strong coupling case, J. Differential Equations 178 (2002), no. 2, 478-518. 
33. _ Asymmetric spotty patterns for the Gray-Scott model in $\mathbf{R}^{2}$, Stud. Appl. Math. 110 (2003), no. 1, 63-102.

34. Existence and stability of multiple-spot solutions for the Gray-Scott model in $\mathbb{R}^{2}$, Phys. D 176 (2003), no. 3-4, 147-180.

35. _ Existence and stability analysis of asymmetric patterns for the Gierer-Meinhardt system, J. Math. Pures Appl. (9) 83 (2004), no. 4, 433-476.

36. - On the Gierer-Meinhardt system with saturation, Commun. Contemp. Math. 6 (2004), no. 2, 259-277.

Department of Mathematics, University of South Florida, Tampa, FL 33620-5700, USA

E-mail address: you@math.usf.edu 